\title{
Synaptic mitochondria are critical for hair-cell synapse formation and function
}

2

Hiu-tung C. Wong ${ }^{1,2}$, Qiuxiang Zhang ${ }^{1}$, Alisha J. Beirl ${ }^{1}$, Ronald S. Petralia ${ }^{3}$, Ya-Xian Wang ${ }^{3}$, Katie S. Kindt ${ }^{1 *}$

${ }^{1}$ Section on Sensory Cell Development and Function

NIDCD/National Institutes of Health

Bethesda, MD 20892 USA

${ }^{2}$ National Institutes of Health-Johns Hopkins University Graduate Partnership Program, NIDCD/National Institutes of Health

Bethesda, MD 20892 USA

${ }^{3}$ Advanced Imaging Core

NIDCD/National Institutes of Health

Bethesda, MD 20892, USA

\section{${ }^{*}$ Corresponding author}

Katie Kindt: katie.kindt@nih.gov

\section{Summary}

Sensory hair cells in the ear utilize specialized ribbon synapses. These synapses are defined by electron-dense presynaptic structures called ribbons, composed primarily of the structural protein Ribeye. Previous work has shown that voltage-gated influx of $\mathrm{Ca}^{2+}$ through Cav1.3 channels is critical for hair-cell synapse function and can impede ribbon formation. We show that in mature zebrafish hair cells, evoked presynaptic-Ca ${ }^{2+}$ influx through Cav1.3 channels initiates mitochondrial- $\mathrm{Ca}^{2+}\left(\right.$ mito- $\left.\mathrm{Ca}^{2+}\right)$ uptake adjacent to ribbons. Block of mito-Ca ${ }^{2+}$ uptake in mature cells depresses presynaptic $\mathrm{Ca}^{2+}$ influx and impacts synapse integrity. In developing zebrafish hair cells, mito- $\mathrm{Ca}^{2+}$ uptake coincides with spontaneous rises in presynaptic $\mathrm{Ca}^{2+}$ influx. Spontaneous mito- $\mathrm{Ca}^{2+}$ loading lowers cellular $\mathrm{NAD}^{+} / \mathrm{NADH}$ redox and downregulates ribbon formation. Direct application of $\mathrm{NAD}^{+}$or $\mathrm{NADH}$ increases or decreases ribbon formation respectively, possibly acting through the $\mathrm{NAD}(\mathrm{H})$-binding domain on Ribeye. Our results present a mechanism where presynaptic- and mito- $\mathrm{Ca}^{2+}$ couple to confer proper presynaptic function and formation.

Keywords: Hair cell, ribbon synapse, mitochondria, $\mathrm{Ca}^{2+}, \mathrm{NAD}(\mathrm{H})$ redox 


\section{Introduction}

Neurotransmission is an energy demanding process that relies heavily on mitochondria.

In neurons, mitochondria dysfunction has been implicated in synaptopathies that impact

41 neurodevelopment, learning and memory, and can contribute to neurodegeneration (Flippo

42 and Strack, 2017; Lepeta et al., 2016; Todorova and Blokland, 2017). In hair cells, sensory

43 neurotransmission relies on specialized ribbon synapses to facilitate rapid and sustained vesicle

44 release that is particularly energy demanding (reviewed in: Johnson et al. 2019;__agnado and

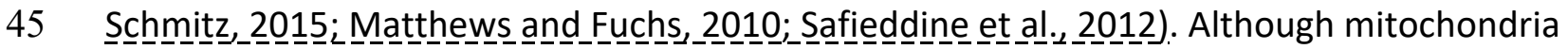

46 dysfunction has been implicated in hearing loss (Böttger and Schacht, 2013; Fischel-Ghodsian et

47 al., 2004; Kokotas et al., 2007), the precise role mitochondria play at hair-cell synapses remains 48 unclear.

Ribbon synapses are characterized by a unique presynaptic structure called a "ribbon" that tethers and stabilizes synaptic vesicles at the active zone (reviewed in: Matthews

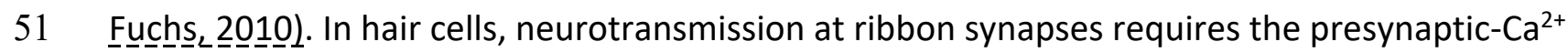

52 channel Cav1.3 (Brandt et al., 2003; Kollmar et al., 1997; Sidi et al., 2004). Hair-cell

53 depolarization opens Cav1.3 channels, resulting in a spatially restricted increase of $\mathrm{Ca}^{2+}$ at

54 presynaptic ribbons that triggers vesicle fusion. Tight spatial regulation of presynaptic $\mathrm{Ca}^{2+}$ is important for ribbon-synapse function and requires efficient $\mathrm{Ca}^{2+}$ clearance through a

56 combination of $\mathrm{Ca}^{2+}$ pumps, $\mathrm{Ca}^{2+}$ buffers and intracellular $\mathrm{Ca}^{2+}$ stores (Carafoli, 2011; Mulkey and Malenka, 1992; Tucker and Fettiplace, 1995; Yamoah et al., 1998; Zenisek and Matthews, 2000). While ER Ca ${ }^{2+}$ stores have been implicated in hair-cell neurotransmission, whether mitochondrial- $\mathrm{Ca}^{2+}\left(\right.$ mito- $\left.\mathrm{Ca}^{2+}\right)$ stores play a role in this process remains unclear (CastellanoMuñoz and Ricci, 2014; Kennedy, 2002; Lioudyno et al., 2004; Tucker and Fettiplace, 1995).

62 also play an important role during inner-ear development. In mammals, prior to hearing onset, 63 auditory hair cells fire spontaneous $\mathrm{Ca}^{2+}$ action potentials (Eckrich et al., 2018; Marcotti et al., 64 2003; Tritsch et al., 2007, 2010). In mammalian hair cells, these $\mathrm{Ca}^{2+}$ action potentials are 65 Cav1.3-dependent and are thought to be important for synapse and circuit formation. In 
support of this idea, in vivo work in zebrafish hair cells found that increasing or decreasing voltage-gated $\mathrm{Ca}^{2+}$ influx through Cav1.3 channels during development led to the formation of smaller or larger ribbons respectively (Sheets et al., 2012). Furthermore, in mouse knockouts of Cav1.3, auditory outer hair cells have reduced afferent innervation and synapse number (Ceriani et al., 2019). Mechanistically, how Cav1.3-channel activity regulates ribbon size and innervation, and whether hair-cell $\mathrm{Ca}^{2+}$ stores play a role in this process is not known.

Cumulative work has shown that ribbon size varies between species and sensory epithelia (reviewed in Moser_et al._.2006); these variations are thought to reflect important encoding requirements of a given sensory cell (Matthews and Fuchs, 2010). In auditory hair cells, excitotoxic noise damage can also alter ribbon size, and lead to hearing deficits (Jensen et al., 2015; Kujawa and Liberman, 2009; Liberman et al., 2015). Excitotoxic damage is thought to be initiated by mito-Ca ${ }^{2+}$ overload and subsequent ROS production (Böttger and Schacht, 2013; Wang et al., 2018). Mechanistically, precisely how ribbon size is established during development or altered under pathological conditions is not fully understood.

One known way to regulate ribbon formation is through its main structural component Ribeye (Schmitz et al., 2000a). Perhaps unsurprisingly, previous work has shown that overexpression or depletion of Ribeye in hair cells can increase or decrease ribbon size respectively (Becker et al., 2018; Jean et al., 2018; Sheets, 2017; Sheets et al., 2011a). Ribeye is a splice variant of the transcriptional co-repressor Carboxyl-terminal binding protein 2 (CtBP2) a splice variant that is unique to vertebrate evolution (Schmitz et al., 2000a). Ribeye contains a unique A-domain, and a B-domain that is nearly identical to full-length CtBP2. The B-domain contains a nicotinamide adenine dinucleotide $\left(\mathrm{NAD}^{+}, \mathrm{NADH}\right.$ or $\left.\mathrm{NAD}(\mathrm{H})\right)$ binding site (Schmitz et al., 2000; Magupalli et al., 2008). NAD(H) redox is linked to mitochondrial metabolism (Srivastava, 2016). Because CtBP is able to bind and detect NAD ${ }^{+}$and NADH levels, it is thought to function as a metabolic biosensor (Stankiewicz et al., 2014). For example, previous work has

91 demonstrated that changes in $\mathrm{NAD}(\mathrm{H})$ redox can impact CtBP oligomerization and its

92 transcriptional activity (Fjeld et al., 2003; Thio et al., 2004). Interestingly, in vitro work has

93 shown that both $\mathrm{NAD}^{+}$and $\mathrm{NADH}$ can also promote interactions between Ribeye domains 
94 (Magupalli et al., 2008). Whether NAD ${ }^{+}$or NADH can impact Ribeye interactions and ribbon

95 formation or stability has not been confirmed in vivo.

96 In neurons, it is well established that during presynaptic activity, mitochondria clear and

97 store $\mathrm{Ca}^{2+}$ at the presynapse (Devine and Kittler, 2018). Additionally, presynaptic activity and

98 mito- $\mathrm{Ca}^{2+}$ can couple together to influence cellular bioenergetics, including $\mathrm{NAD}(\mathrm{H})$ redox

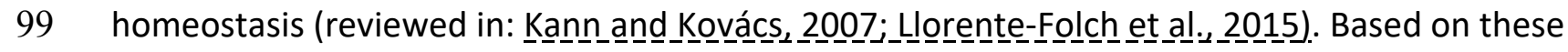

100 studies, we hypothesized that $\mathrm{Ca}^{2+}$ influx through Cav1.3 channels may regulate mito-Ca ${ }^{2+}$,

101 which in turn could regulate $\operatorname{NAD}(H)$ redox. Changes to cellular bioenergetics and $\operatorname{NAD}(H)$ redox

102 could function to control Ribeye interactions and ribbon formation or impact ribbon-synapse

103 function and stability.

104 To study the impact of mito- $\mathrm{Ca}^{2+}$ and $\mathrm{NAD}(\mathrm{H})$ redox on ribbon synapses, we examined

105 hair cells in the lateral-line system of larval zebrafish. This system is advantageous for our

106 studies because it contains hair cells with easy access for in vivo pharmacology, mechanical

107 stimulation and imaging cellular morphology and function. Within the lateral-line, hair cells are

108 arranged in clusters called neuromasts. The hair cells and ribbon synapses in each cluster form

109 rapidly between 2 to 3 days post-fertilization (dpf) but by 5-6 dpf, the majority of hair cells are

110 mature, and the system is functional (Kindt et al., 2012; McHenry et al., 2009; Metcalfe, 1985;

111 Murakami et al., 2003; Santos et al., 2006). Thus, these two ages (2-3 dpf and 5-6 dpf) can be

112 used to study mito- $\mathrm{Ca}^{2+}$ and $\mathrm{NAD}(\mathrm{H})$ redox in developing and mature hair cells respectively.

113 Using this sensory system, we find that presynaptic $\mathrm{Ca}^{2+}$ influx drives mito-Ca ${ }^{2+}$ uptake.

114 In mature hair cells, mito- $\mathrm{Ca}^{2+}$ uptake occurs during evoked stimulation and is required to

115 sustain presynaptic function and ultimately synapse integrity. In developing hair cells, mito-Ca ${ }^{2+}$

116 uptake coincides with spontaneous rises in presynaptic $\mathrm{Ca}^{2+}$. Blocking these spontaneous

117 changes in $\mathrm{Ca}^{2+}$ leads to the formation of larger ribbons. Using a redox biosensor, we

118 demonstrate that specifically in developing hair cells, decreasing mito-Ca ${ }^{2+}$ levels increases the

$119 \mathrm{NAD}^{+} / \mathrm{NADH}$ redox ratio. Furthermore, we show that application of $\mathrm{NAD}^{+}$or NADH can increase

120 or decrease ribbon formation respectively. Overall our results suggest that in hair cells

121 presynaptic $\mathrm{Ca}^{2+}$ influx and mito- $\mathrm{Ca}^{2+}$ uptake couple to impact ribbon formation and function. 


\section{Results}

\section{Mitochondria are located near presynaptic ribbons}

In neurons, synaptic mitochondria have been shown to influence synapse formation,

127 plasticity and function (Flippo and Strack, 2017; Todorova and Blokland, 2017). Based on this

128 work, we hypothesized that mitochondria may impact synapses in hair cells. Therefore, we

129 examined the proximity of mitochondria relative to presynaptic ribbons in zebrafish lateral-line

130 hair cells. We visualized mitochondria and ribbons using transmission electron microscopy

131 (TEM) and in live hair cells using Airyscan confocal microscopy.

132 Using TEM, we examined sections that clearly captured ribbons (Example, Figure 1C).

133 We were able to observe a mitochondrion in close proximity $(<1 \mu \mathrm{m})$ to ribbons in $74 \%$ of the

134 sections (Figure 1D, median ribbon-to-mitochondria distance $=174 \mathrm{~nm}, \mathrm{n}=17$ out of 21

135 sections). To obtain a more comprehensive understanding of the 3D morphology and location

136 of mitochondria relative to ribbons in live cells, we used Airyscan confocal microscopy. To

137 visualize these structures in living cells, we used transgenic zebrafish expressing MitoGCaMP3

138 (Esterberg et al., 2014) and Ribeye a-tagRFP (Sheets et al., 2017) in hair cells to visualize

139 mitochondria and ribbons respectively. Using this approach, we observed tubular networks of

140 mitochondria extending from apex to base (Figure 1A-B, E-E', Figure S1A, Movie S1). At the base

141 of the hair cell, we observed ribbons nestled between branches of mitochondria. Overall our

142 TEM and Airyscan imaging suggests that in lateral-line hair cells, mitochondria are present near

143 ribbons and are poised to impact ribbon synapses.

Mito- $\mathrm{Ca}^{2+}$ uptake at ribbons is MCU and Cav1.3 dependent

In zebrafish hair cells, robust rises in mito- $\mathrm{Ca}^{2+}$ have be reported during mechanical

147 stimulation (Pickett et al., 2018). Due to the proximity of the mitochondria to the ribbon, we

148 predicted that rises in mito- $\mathrm{Ca}^{2+}$ levels during mechanical stimulation are related to presynapse-

149 associated rises in $\mathrm{Ca}^{2+}$.

150 To test this prediction, we used a fluid-jet to mechanically stimulate hair cells and evoke

151 presynaptic activity. During stimulation, we used MitoGCaMP3 to monitor mito-Ca ${ }^{2+}$ in hair 
152 cells. As previously reported, we observed robust mito- $\mathrm{Ca}^{2+}$ uptake during stimulation (Figure

153 1E-F). We examined the subcellular distribution of MitoGCaMP3 signals over time and found

154 that the signals initiated near ribbons (Figure 1E). During the latter part of the stimulus, and

155 even after the stimulus terminated, the MitoGCaMP3 signals propagated apically within the

156 mitochondria, away from the ribbons (Example, Figure 1E-E", regions 1-3). We characterized

157 the time course of MitoGCaMP3 signals with regards to onset kinetics and return to baseline.

158 During a 2-s stimulus, we detected a significant rise in MitoGCaMP3 signals $0.6 \mathrm{~s}$ after stimulus

159 onset (Figure S1B). Interestingly, after the stimulus terminated, MitoGCaMP3 levels took

160 approximately $5 \mathrm{~min}$ to return to baseline (Figure S1C-C'). As previously reported, the kinetics

161 of MitoGCaMP3 signals in hair cells mitochondria were quite different from signals observed

162 using cytosolic GCaMP3 (CytoGCaMP3) in hair cells (Pickett et al., 2018). Compared to

163 MitoGCaMP3 signals, CytoGCaMP3 signals had faster onset kinetics, and a faster return to

164 baseline (Figure S1B-C, time to rise: $0.06 \mathrm{~s}$, post-stimulus return to baseline: $12 \mathrm{~s}$ ). These

165 differences in kinetics indicate that mito- $\mathrm{Ca}^{2+}$ loading operates over slower timescales

166 compared to the cytosolic compartment. It also confirms that hair-cell stimulation can initiate

167 long lasting increases in mito-Ca ${ }^{2+}$.

To verify that MitoGCaMP3 signals reflect $\mathrm{Ca}^{2+}$ entry into mitochondria, we applied

169 Ru360, an antagonist of the mito- $\mathrm{Ca}^{2+}$ uniporter (MCU). The MCU is the main pathway for rapid

$170 \mathrm{Ca}^{2+}$ entry into the mitochondria (Matlib et al., 1998). We found that stimulus-evoked

171 MitoGCaMP3 signals were blocked in a dose-dependent manner after treatment with Ru360

172 (Figure $1 F)$. Due to the initiation of mito- $\mathrm{Ca}^{2+}$ near ribbons, we examined whether presynaptic

$173 \mathrm{Ca}^{2+}$ influx through Cav1.3 channels was the main source of $\mathrm{Ca}^{2+}$ entering the mitochondria. To

174 examine Cav1.3 channel contribution to mito-Ca ${ }^{2+}$ uptake, we applied isradipine, a Cav1.3

175 channel antagonist. Similar to blocking the MCU, blocking Cav1.3 channels eliminated all

176 stimulus-evoked MitoGCaMP3 signals (Figure 1F). Overall our MitoGCaMP3 functional imaging

177 indicates that in hair cells, evoked mito- $\mathrm{Ca}^{2+}$ uptake initiates near ribbons and is dependent on

178 MCU and Cav1.3 channel function. 
Interestingly, we observed that mito-Ca ${ }^{2+}$ uptake was only present in $\sim 40 \%$ of cells

182 (Example, Figure 2A'; $n=10$ neuromasts, 146 cells). This observation is consistent with previous

183 work demonstrating that only $\sim 30 \%$ of hair cells within each neuromast cluster have

184 presynaptic $\mathrm{Ca}^{2+}$ signals and are synaptically active (Zhang et al., 2018b). Because presynaptic

$185 \mathrm{Ca}^{2+}$ signals initiate near mitochondria, it is probable that mito-Ca ${ }^{2+}$ uptake may occur

186 specifically in hair cells with synaptic activity.

187 To test whether evoked mito- $\mathrm{Ca}^{2+}$ uptake occurred exclusively in cells with presynaptic

$188 \mathrm{Ca}^{2+}$ influx, we performed two-color functional imaging. We used a double transgenic approach

189 that utilized a membrane-localized GCaMP6s (GCaMP6sCAAX; green) to measure presynaptic

$190 \mathrm{Ca}^{2+}$ signals at the base of hair cells (Jiang et al., 2017a; Sheets et al., 2017), and concurrently

191 used MitoRGECO1 (red) to examine mito-Ca ${ }^{2+}$ signals (Figure 2A-B'). Our two-color imaging

192 approach revealed a strong correlation between the magnitude of the GCaMP6sCAAX and

193 MitoRGECO1 signals (Figure $2 B, R^{2}=0.8, p<0.0001 ; n=209$ cells). We found that the median

194 MitoRGECO1 signals were $400 \%$ larger in presynaptically active hair cells compared to

195 presynaptically silent hair cells (Figure $2 \mathrm{~B}^{\prime}$ ). Together these results suggest that mito-Ca ${ }^{2+}$

196 uptake occurs specifically in hair cells with evoked presynaptic-Ca ${ }^{2+}$ influx.

\section{Blocking Mito- $\mathrm{Ca}^{2+}$ entry depresses presynaptic $\mathrm{Ca}^{2+}$ signals in mature hair cells}

Although we observed mito-Ca ${ }^{2+}$ uptake specifically in hair cells with active $\mathrm{Ca}^{2+}$

200 channels, the impact of mito- $\mathrm{Ca}^{2+}$ uptake on the function of hair-cell synapses was unclear.

201 Based on previous studies in neurons (Billups and Forsythe, 2002; Levy et al., 2003; Chouhan et

202 al., 2010; Kwon et al., 2016), we reasoned that mitochondria may also be important to remove

203 excess $\mathrm{Ca}^{2+}$ from the hair-cell presynapse to regulate neurotransmission.

204 To determine if mito-Ca ${ }^{2+}$ uptake impacted presynaptic function, we assayed evoked

205 presynaptic-Ca ${ }^{2+}$ signals by monitoring GCaMP6sCAAX signals adjacent to ribbons as described

206 previously (Example, Figure S2, Sheets et al., 2017; Zhang et al., 2018b). We examined

207 GCaMP6sCAAX signals in mature-hair cells at 5-6 dpf when neuromast organs are largely

208 mature (Kindt et al., 2012; McHenry et al., 2009; Metcalfe, 1985; Murakami et al., 2003; Santos

209 et al., 2006). Using this approach, we assayed presynaptic GCaMP6sCAAX signals before and 
210 after a 20-min application of the MCU antagonist Ru360 (Figure 2C-D'). We found that during

211 short, 200-ms stimuli, GCaMP6sCAAX signals at ribbons were reduced after complete MCU

212 block (10 $\mu$ M Ru360, Figure 2C-C'). Reduction of GCaMP6sCAAX signals were further

213 exacerbated during sustained 10-s stimuli, even when the MCU was only partially blocked (2

$214 \mu \mathrm{M}$ Ru360, Figure 2D-D'). These results suggest that in mature hair cells, evoked mito-Ca ${ }^{2+}$

215 uptake is critical for presynaptic $\mathrm{Ca}^{2+}$ influx, especially during sustained stimulation.

\section{Evoked mito- $\mathrm{Ca}^{2+}$ uptake is important for mature synapse integrity and cell health}

218 MCU block could impair presynaptic $\mathrm{Ca}^{2+}$ influx through several mechanisms. It could

219 impair the biophysical properties of Cav1.3 channels, for example, through $\mathrm{Ca}^{2+}$-dependent

220 inactivation (Platzer et al., 2000; Schnee and Ricci, 2003). In addition, mito-Ca ${ }^{2+}$ has been

221 implicated in synapse dysfunction and cell death (Esterberg et al., 2014; Vos et al., 2010; Wang

222 et al., 2018), and MCU block could be pathological. To distinguish between these possibilities,

223 we assessed whether synapse or hair-cell number were altered after MCU block with Ru360.

224 To quantify ribbon-synapse morphology after MCU block, we immunostained mature-

225 hair cells (5 dpf) with Ribeye $b$ and MAGUK antibodies to label presynaptic ribbons and

226 postsynaptic densities (MAGUK) respectively. We first applied $2 \mu \mathrm{M}$ Ru360 for $1 \mathrm{hr}$, a

227 concentration that partially reduces evoked mito-Ca ${ }^{2+}$ uptake (See Figure $1 \mathrm{~F}^{\prime}$ ) yet is effective at

228 reducing sustained presynaptic $\mathrm{Ca}^{2+}$ influx (See Figure 2D-D'). At this dose, Ru360 had no impact

229 on hair cell or synapse number (Figure 3E). In addition, we observed no morphological change

230 in ribbon or postsynapse size (Figure 3F, Figure S3A). These findings indicate that partial MCU

231 block can impair presynaptic function without any observable pathology.

232 We also tested a higher dose of Ru360 (10 $\mu \mathrm{M})$ that completely blocks evoked mito-Ca ${ }^{2+}$

233 uptake (See Figure 1F). Interestingly, a 30-min or 1-hr $10 \mu \mathrm{M}$ Ru360 treatment had a

234 progressive impact on synapse and cellular integrity. After a 30-min treatment with $10 \mu \mathrm{M}$

235 Ru360 we observed significantly fewer complete synapses per hair cell, but not fewer hair cells

236 compared to controls (Figure 3E; Hair cells per neuromast, control: 16.3, 30-min $10 \mu \mathrm{M}$ Ru360:

237 15.5; $p=0.5)$. In addition, after the 30-min treatment, ribbons were significantly larger (Figure

238 3F). The pathological effects of MCU block were more pronounced after a 1-hr, $10 \mu \mathrm{M}$ Ru360 
treatment. After 1-hr, there was both fewer hair cells per neuromast (Hair cells per neuromast, control: 18.1, 1-hr 10 MM Ru360: 12.0; p >0.0001) and fewer synapses per hair cell (Figure 3E).

241 Similar to 30-min treatments with Ru360, after $1 \mathrm{hr}$, ribbons were also significantly larger

242 (Figure 3F). Neither 30-min nor 1-hr $10 \mu \mathrm{M}$ Ru360 treatment altered postsynapse size (Figure

243 S3A). Overall, our results indicate that in mature hair cells, partial block of mito-Ca ${ }^{2+}$ uptake can

244 impair presynaptic function without altering presynaptic morphology or synapse integrity.

245 Complete block of mito- $\mathrm{Ca}^{2+}$ uptake is pathological; it impairs presynaptic function, alters

246 presynaptic morphology, and results in a loss of synapses and hair-cells.

Spontaneous presynaptic and mito- $\mathrm{Ca}^{2+}$ influx pair in developing hair cells

In addition to evoked presynaptic- and mito- $\mathrm{Ca}^{2+}$ signals in hair cells, we also observed instances of spontaneous presynaptic- and mito-Ca ${ }^{2+}$ signals (Example, Figure 4A-A"', , Movie S2). Numerous studies have demonstrated that mammalian hair cells have spontaneous presynaptic- $\mathrm{Ca}^{2+}$ influx during development (Eckrich et al., 2018; Marcotti et al., 2003; Tritsch et al., 2007, 2010). Therefore, we predicted that similar to mammals, spontaneous presynaptic$\mathrm{Ca}^{2+}$ uptake may be a feature of development. Furthermore, we predicted that spontaneous mito- $\mathrm{Ca}^{2+}$ uptake may correlate with instances of spontaneous presynaptic- $\mathrm{Ca}^{2+}$ influx.

First we tested whether spontaneous presynaptic- $\mathrm{Ca}^{2+}$ signals were a feature of development. In zebrafish neuromasts, hair cells are rapidly added between 2-3 dpf, but by 5-6 dpf relatively fewer cells are added and the hair cells and the organs are largely mature (Kindt et al., 2012; McHenry et al., 2009; Metcalfe, 1985; Murakami et al., 2003; Santos et al., 2006).

260 Therefore, we examined the magnitude and frequency of spontaneous, presynaptic

261 GCaMP6sCAAX signals in developing ( $3 \mathrm{dpf}$ ) and mature hair cells ( $5 \mathrm{dpf}$ ). We found that in

262 developing hair cells, spontaneous GCaMP6sCAAX signals occurred with larger magnitudes and 263 more frequency compared to those in mature hair cells (Figure 4B-C). Our spontaneous

264 GCaMP6sCAAX imaging demonstrates that similar to mammals, spontaneous presynaptic $\mathrm{Ca}^{2+}$ 265 activity is a feature of developing zebrafish hair cells.

266 Next, we tested whether spontaneous mito-Ca ${ }^{2+}$ uptake and presynaptic-Ca ${ }^{2+}$ influx 267 were correlated. For this analysis we concurrently imaged GCaMP6sCAAX and MitoRGECO1 
268 signals in the same cells for 15 mins to measure presynaptic and mito-Ca ${ }^{2+}$ responses respectively. We found that spontaneous presynaptic-Ca ${ }^{2+}$ influx was often associated with

270 spontaneous mito-Ca ${ }^{2+}$ uptake (Example, Figure $\left.4 A-A^{\prime \prime \prime}\right)$. Overall, we observed a high

271 correlation between the rise and fall of these two signals within individual cells (Figure $A^{\prime \prime}-A^{\prime \prime \prime}$ ).

272 Both of these signals and their correlation are abolished by application of the Cav1.3-channel

273 antagonist isradipine (Figure S4). Together these experiments indicate that, similar to our

274 evoked experiments, spontaneous presynaptic- and mito- $\mathrm{Ca}^{2+}$ signals are correlated.

\section{Spontaneous mito- $\mathrm{Ca}^{2+}$ uptake regulates ribbon formation}

Previous work in zebrafish demonstrated that Cav1.3 channel activity plays a role in

278 ribbon formation specifically during development (Sheets et al., 2012). This work found that a

279 transient, 1-hr pharmacological block of Cav1.3 channels increased ribbon size, while Cav1.3

280 channel agonists decreased ribbon size (Figure 5E; Sheets et al., 2012). Therefore, spontaneous

$281 \mathrm{Cav1.3}$ and $\mathrm{MCU} \mathrm{Ca}^{2+}$ activities could function together to control ribbon size in developing hair 282 cells.

To characterize the role of MCU function and spontaneous mito-Ca ${ }^{2+}$ uptake on ribbon formation, we applied the MCU antagonist Ru360 to developing hair cells ( $3 \mathrm{dpf})$. After this treatment, we quantified ribbon synapse morphology by immunostaining hair cells to label presynaptic ribbons and postsynaptic densities. After a 1-hr application of $2 \mu \mathrm{M}$ Ru360 to block the MCU, we observed a significant increase in ribbon size in developing hair cells (Figure 5A-B, E). In contrast, this same treatment did not impact ribbon size in mature hair cells (Figure 3F). We also applied a higher concentration of Ru360 (10 $\mu \mathrm{M})$ to developing hair cells for $1 \mathrm{hr}$. In

290 developing hair cells, after a 1-hr $10 \mu \mathrm{M}$ Ru360 treatment, we also observed a significant 291 increase in ribbon size (Figure 5A, C, E). Unlike in mature hair cells (Figure 3), in developing hair 292 cells, these concentrations of the MCU antagonist did not alter the number of hair cells, nor the 293 number of synapses per hair cell (Figure 5D; Hair cells per neuromast, control: 9.0, 1-hr $10 \mu \mathrm{M}$ 294 Ru360: 8.8, $p=0.3$ ). All morphological changes were restricted to the ribbons, as MCU block did 295 not alter the size of the postsynapse (Figure S3C). 
In addition to larger ribbons, at higher concentrations of Ru360 (10 $\mu \mathrm{M})$ we also

297 observed an increase in cytoplasmic, non-synaptic Ribeye aggregates (Figure 5F, G). Previous

298 work in zebrafish reported both larger ribbons and cytoplasmic aggregates of Ribeye in Cav1.3a-

299 deficient hair cells (Sheets et al., 2011a). These parallel phenotypes indicate that spontaneous

300 presynaptic $\mathrm{Ca}^{2+}$ influx and mito- $\mathrm{Ca}^{2+}$ uptake may couple to shape ribbon formation. Our results

301 suggest that during development, spontaneous $\mathrm{Ca}^{2+}$ entry through both $\mathrm{Cav} 1.3$ and MCU

302 channels continuously regulate ribbon formation; blocking either channel increases Ribeye

303 aggregation and ribbon size.

MCU and Cav1.3 channel activities regulate subcellular $\mathrm{Ca}^{2+}$ homeostasis

Our results indicate that spontaneous $\mathrm{Ca}^{2+}$ influx through Cav1.3 channels and

307 subsequent loading of $\mathrm{Ca}^{2+}$ into mitochondria regulates ribbon formation in developing hair

308 cells. But how do these two $\mathrm{Ca}^{2+}$ signals converge to regulate ribbon formation? It is possible

309 that mitochondria could buffer $\mathrm{Ca}^{2+}$ during spontaneous presynaptic activity and function to

310 decrease resting levels of cytosolic $\mathrm{Ca}^{2+}$ (cyto-Ca ${ }^{2+}$ ); cyto-Ca ${ }^{2+}$ levels could be a signal that

311 regulates ribbon formation. To examine resting cyto- $\mathrm{Ca}^{2+}$ levels in hair cells, we examined the

312 fluorescence signal change of the cytosolic $\mathrm{Ca}^{2+}$ indicator RGECO1 (CytoRGECO1) before and

313 after a 30-min pharmacological manipulation of Cav1.3 or MCU channels (Figure 6A-C).

314 We observed that treatment with the Cav1.3 channel antagonist isradipine and agonist

315 Bay K8644 decreased and increased resting CytoRGECO1 fluorescence respectively (Figure 6B).

316 However, treatment with MCU blocker Ru360 did not significantly shift resting CytoRGECO1

317 fluorescence levels (Figure 6B). Similar results with Ru360 were observed in developing and

318 mature hair cells (Figure 6B-C). These data suggest that, unlike Cav1.3 channel function, MCU

319 function and associated mito- $\mathrm{Ca}^{2+}$ uptake does not play a critical role in buffering steady state 320 cyto-Ca ${ }^{2+}$ levels.

$321 \quad$ Alternatively, it is possible that rather than impacting cyto-Ca ${ }^{2+}$ levels, both Cav1.3 and

$322 \mathrm{MCU}$ activity are required to load and maintain $\mathrm{Ca}^{2+}$ levels within the mitochondria. In this

323 scenario, mito- $\mathrm{Ca}^{2+}$ levels could be a signal that regulates ribbon formation. To test this

324 possibility, we used MitoGCaMP3 to examine resting mito-Ca ${ }^{2+}$ levels before and after 
325 modulating Cav1.3 or MCU channel function (Figure 6D-F). We observed that blocking Cav1.3

326 channels with isradipine or the MCU with Ru360 decreased resting MitoGCaMP3 fluorescence

327 (Figure 6E-F). Conversely, Cav1.3 channel agonist Bay K8644 increased resting MitoGCaMP3

328 fluorescence (Figure 6E). These results were consistent in developing and mature hair cells

329 (Figure 6E-F). Our resting MitoGCaMP3 measurements indicate that the effects of Cav1.3

330 channel and MCU activity converge in to regulate mito- $\mathrm{Ca}^{2+}$ levels. When either of these

331 channels are blocked, the resting levels of mito- $\mathrm{Ca}^{2+}$ are decreased. Therefore, if presynaptic

$332 \mathrm{Ca}^{2+}$ influx and mito-Ca ${ }^{2+}$ regulate ribbon formation through a similar mechanism, they may act

333 through mito- rather than cyto- $\mathrm{Ca}^{2+}$ homeostasis.

\section{Mito- $\mathrm{Ca}^{2+}$ levels regulate $\mathrm{NAD}(\mathrm{H})$ redox in developing hair cells}

If mito- $\mathrm{Ca}^{2+}$ levels signal to regulate ribbon formation, how is this signal transmitted

337 from the mitochondria to the ribbon? An ideal candidate is via NAD $(H)$ homeostasis. Ribeye

338 protein, the main component of ribbons contains a putative $N A D(H)$ binding site. Because

339 mitochondria regulate $N A D(H)$ redox homeostasis (Jensen-Smith et al., 2012) we reasoned that

340 there may be a relationship between mito-Ca ${ }^{2+}$ levels, NAD(H) redox and ribbon formation.

341 To examine $\mathrm{NAD}(\mathrm{H})$ redox, we created a stable transgenic line expressing Rex-YFP, a

342 fluorescent $\mathrm{NAD}^{+} / \mathrm{NADH}$ ratio biosensor in hair cells (Figure 6G). We verified the function of the

343 Rex-YFP biosensor in our in vivo system by exogenously applying NAD ${ }^{+}$or NADH for $30 \mathrm{~min}$. We

344 found that incubations with $100 \mu \mathrm{M} \mathrm{NAD}^{+}$increased while $5 \mathrm{mM}$ NADH decreased Rex-YFP

345 fluorescence; these intensity changes are consistent with an increase and decrease in the

$346 \mathrm{NAD}^{+} / \mathrm{NADH}$ ratio respectively (Figure $6 \mathrm{H}$ ). Next, we examined if Cav1.3 and MCU channel

347 activities impact the $\mathrm{NAD}^{+} / \mathrm{NADH}$ ratio. We found that 30-min treatments with either Cav1.3 or

348 MCU channel antagonist increased the NAD ${ }^{+}$NADH ratio (increased Rex-YFP fluorescence) in

349 developing hair cells (Figure 6H). Interestingly, similar 30-min treatments did not alter Rex-YFP

350 fluorescence in mature hair cells (Figure 6I). Together, our baseline MitoGCaMP3 and Rex-YFP

351 measurements indicate that during development, Cav1.3 and MCU channel activities normally

352 function to increase mito- $\mathrm{Ca}^{2+}$ and decrease the $\mathrm{NAD}^{+} / \mathrm{NADH}$ ratio. Overall, this work provides

353 strong evidence that links $\mathrm{NAD}(\mathrm{H})$ redox and mito- $\mathrm{Ca}^{2+}$ with ribbon formation. 


\section{$\mathrm{NAD}^{+}$and NADH directly influence ribbon formation}

Our Rex-YFP measurements suggest that spontaneous Cav1.3 and $\mathrm{MCU} \mathrm{Ca}^{2+}$ activities

normally function to decrease the $\mathrm{NAD}^{+} / \mathrm{NADH}$ ratio; furthermore, this activity may function to restrict ribbon formation. Conversely, blocking these activities increases the $\mathrm{NAD}^{+} / \mathrm{NADH}$ ratio and may increase ribbon formation. If the $\mathrm{NAD}^{+} / \mathrm{NADH}$ ratio is an intermediate step between Cav1.3 and MCU channel activities and ribbon formation, we predicted that more $\mathrm{NAD}^{+}$or NADH would increase or decrease ribbon formation respectively. To test this prediction, we treated developing hair cells with exogenous $\mathrm{NAD}^{+}$or $\mathrm{NADH}$.

After a 1-hr treatment with $100 \mu \mathrm{M} \mathrm{NAD}^{+}$, we found that the ribbons in developing hair cells were significantly larger compared to controls (Figure 7A-B, E). In contrast, after a 1-hr treatment with $5 \mathrm{mM} \mathrm{NADH}$, ribbons were significantly smaller compared to controls (Figure

$3667 \mathrm{~A}, \mathrm{C}, \mathrm{E})$. Neither exogenous $\mathrm{NAD}^{+}$nor $\mathrm{NADH}$ were able to alter ribbon size in mature hair cells

367 (Figure 7F-H, J). These concentrations of $\mathrm{NAD}^{+}$and $\mathrm{NADH}$ altered neither the number of 368 synapses per hair cell nor postsynapse size in developing or mature hair cells (Figure 3D, I, 369 Figure S3B, D). These results suggest that in developing hair cells, NAD promotes while NADH 370 inhibits Ribeye-Ribeye interactions or Ribeye localization to the ribbon. Overall these results 371 support the idea that during development, the levels of $\mathrm{NAD}^{+}$and $\mathrm{NADH}$ can directly regulate 372 ribbon formation in vivo.

\section{Discussion}

In this study, we determined in a physiological setting how mito-Ca ${ }^{2+}$ influences hair-cell

376 presynapse function and formation. In mature hair cells, evoked Cav1.3-channel Ca ${ }^{2+}$ influx

377 drives $\mathrm{Ca}^{2+}$ into mitochondria. Evoked mito-Ca ${ }^{2+}$ uptake is important to sustain presynaptic $\mathrm{Ca}^{2+}$

378 responses and maintain synapse integrity (Figure 8B). During development, spontaneous Cav1.3

379 channel $\mathrm{Ca}^{2+}$ influx also drives $\mathrm{Ca}^{2+}$ into mitochondria. Elevated mito-Ca ${ }^{2+}$ levels rapidly lower

380 the $\mathrm{NAD}^{+} / \mathrm{NADH}$ ratio and downregulate ribbon formation (Figure $8 \mathrm{~A}$ ). Furthermore, during

381 development, $\mathrm{NAD}^{+}$and $\mathrm{NADH}$ can directly increase and decrease ribbon formation 
382

383

384

385

386

respectively. Our study reveals an intriguing mechanism that couples presynaptic activity with mito- $\mathrm{Ca}^{2+}$ to regulate the function and formation of a presynaptic structure.

\section{Functional significance of ribbon size}

Our work outlines how presynaptic activity controls the formation and ultimately the size of ribbons. When either presynaptic $\mathrm{Ca}^{2+}$ influx or mito- $\mathrm{Ca}^{2+}$ uptake was perturbed, ribbons were significantly larger (Figure 5A-C, E; Sheets et al.__2012). But why regulate ribbon size?

Previous work has reported variations in ribbon size and shape among hair-cell types and species (Moser et al., 2006). Although ribbon morphology is predicted to impact synapse function, the functional consequence of presynapse structure on function has primarily been explored in the auditory inner hair cells of mice. In these auditory hair cells, studies have identified two distinct populations of ribbon synapses that spatially segregate within each cell (Kalluri and Monges-Hernandez, 2017; Liberman and Liberman, 2016; Liberman et al., 2011; Yin et al., 2014; Zhang et al., 2018a). Structurally, one population has smaller ribbons, while the other population has significantly larger ribbons. Functionally, compared to smaller ribbons, larger ribbons are associated with afferent fibers with less spontaneous activity and higher thresholds of activation (Furman et al._,2013; Kalluri and Monges-Hernandez_ 2017; Liberman et

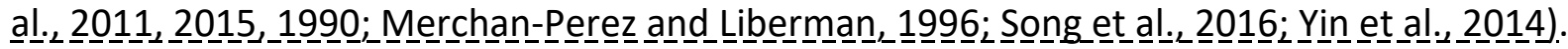
Overall, the combined use of these two types of ribbon synapse is thought to increase the range of sensitivities for each individual auditory hair cell (Costalupes et al., 1984; Ohn et al., 2016). Interestingly, in mice these two populations of ribbons can be distinguished structurally just after the onset of hearing (Liberman and Liberman, 2016). This timing suggests that similar to our data (Figure 4-5), activity during development may help determine ribbon size.

Previous work in the zebrafish-lateral line has also examined how ribbon size impacts synapse function (Sheets et al., 2017). This work overexpressed Ribeye in zebrafish-hair cells to dramatically enlarge ribbons. Functionally, compared to controls, hair cells with enlarged ribbons were associated with afferent neurons with lower spontaneous activity (Sheets et al., 2017). Furthermore, the onset encoding, or the timing of the first afferent spike upon stimulation, was significantly delayed in hair cells with enlarged ribbons. Together, both studies 
411 in zebrafish and mouse indicate that ribbon size can impact the functional properties of the

412 synapse. Based on these studies, we predict that the alterations to ribbon size we observed in

413 our current study would impact functional properties of the synapse in a similar manner. For

414 example, pharmacological treatments that enlarge ribbons (Figure 5: MCU channel block;

415 Figure 7: exogenous $\mathrm{NAD}^{+}$) would also lower spontaneous spiking in afferents and delay onset

416 encoding.

\section{Ribeye and CtBP localization at synapses}

419 In this study, we found that $\mathrm{NAD}(\mathrm{H})$ redox state had a dramatic effect on ribbon

420 formation. $\mathrm{NAD}^{+}$promotes, while NADH reduces ribbon formation (Figure 7). The main

421 component of ribbons is Ribeye. Ribeye has two domains, a unique A domain and a B domain

422 that contains an NAD(H) binding domain (Schmitz et al., 2000a). In vitro work on isolated A and

423 B domains has shown that both $\mathrm{NAD}^{+}$and NADH can affect interactions between $\mathrm{A}$ and $\mathrm{B}$

424 domains as well as B-domain interactions (Magupalli et al., 2008). In the context of ribbons, the

425 B domain has been shown to concentrate at the interface between the ribbon and the

426 membrane opposing the postsynapse (Sheets et al., 2014). Therefore, promoting B domain

427 homodimerization may act to seed larger ribbons at the presynapse. In this scenario, NAD ${ }^{+}$and

$428 \mathrm{NADH}$ could increase and decrease B domain homodimerization to impact ribbon formation.

429 Because we also saw an increase in cytoplasmic Ribeye aggregates after MCU block (Figure 5F-

$430 \mathrm{G})$ it is alternatively possible that $\mathrm{NAD}^{+}$and NADH could impact interactions between $\mathrm{A}$ and $\mathrm{B}$

431 domains to more broadly impact Ribeye interactions and accumulation.

432 Regardless of the exact mechanism, the effect of presynaptic activity and related

433 changes in $\mathrm{NAD}(\mathrm{H})$ redox homeostasis may extend beyond the sensory ribbon synapse. Ribeye

434 is a splice variant of the transcriptional co-repressor CtBP2 (Schmitz et al., 2000b). While the A

435 domain is unique to Ribeye, the B domain is nearly identical to CtBP2 minus the nuclear

436 localization sequence (NLS) (Hübler et al., 2012). In vertebrates, the CtBP family also includes

437 CtBP1 (Chinnadurai, 2007). CtBP proteins are expressed in both hair cells and the nervous

438 system, and there is evidence that both CtBP1 and CtBP2 may act as scaffolds at neuronal

439 synapses (Hübler et al., 2012; tom Dieck et al., 2005). Interestingly, in cultured neurons, it has 
been shown that synaptic activity is associated with both an increase in CtBP1 localization at

441 the presynapse, as well as a decrease in the NAD $/$ NADH ratio (Ivanova et al., 2015). In our in

442 vivo study, we also found that the NAD ${ }^{+}$NADH ratio was lower in developing hair cells with

443 presynaptic activity (Figure 6H). But in contrast to in vitro work on CtBP1 in cultured neurons,

444 we found that Ribeye localization to the presynapse and ribbon size was reduced when the

$445 \mathrm{NAD}^{+} / \mathrm{NADH}$ ratio was lowered (Figure $7 \mathrm{~A}-\mathrm{C}$ ). It is unclear why presynaptic activity regulates

446 Ribeye localization differently from that of CtBP1. Ribeye and CtBP1 behavior may differ due to

447 the divergent function of their N-terminal domains. Synaptic localization may also be influenced

448 by external factors, such as the cell type in which the synapse operates, whether the study is

449 performed in vitro or in vivo, as well as the maturity of the synapse. Overall, both studies

450 demonstrate that CtBP1 and Ribeye localization to the presynapse can be influenced by

451 synaptic activity and $\mathrm{NAD}(\mathrm{H})$ redox state.

Role of evoked mito- $\mathrm{Ca}^{2+}$ uptake in mature hair cells

Studies in various neuronal subtypes have demonstrated that mitochondria play

multiple roles to maintain neurotransmission including ATP production, $\mathrm{Ca}^{2+}$ buffering and

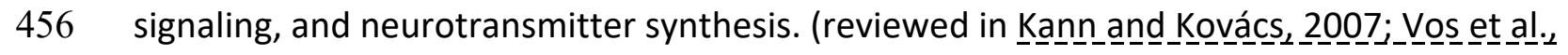

457 2010). Our study found that in mature zebrafish-hair cells, even partially blocking evoked mito-

$458 \mathrm{Ca}^{2+}$ uptake can impair presynaptic $\mathrm{Ca}^{2+}$ influx during sustained stimuli (Figure 2E-F). But how

459 does mito- $\mathrm{Ca}^{2+}$ uptake impact presynaptic $\mathrm{Ca}^{2+}$ activity? Although mito-Ca ${ }^{2+}$ uptake could

460 function to buffer cyto- $\mathrm{Ca}^{2+}$ to maintain presynaptic function, our current work indicates that

461 blocking mito-Ca ${ }^{2+}$ uptake does not raise cytosolic $\mathrm{Ca}^{2+}$ levels (Figure 6A-C). Therefore mito-Ca ${ }^{2+}$

462 uptake may not be required to buffer or clear $\mathrm{Ca}^{2+}$ from the cytosol during steady-state.

463 Alternatively, mito- $\mathrm{Ca}^{2+}$ uptake could buffer $\mathrm{Ca}^{2+}$ locally during presynaptic activity to prevent

$464 \mathrm{Ca}^{2+}$-dependent inactivation of Cav1.3 channels. In hair cells, Cav1.3 channels exhibit reduced

$465 \mathrm{Ca}^{2+}$ dependent inactivation (Koschak et al., 2001; Platzer et al., 2000; Song et al., 2003; Xu and

466 Lipscombe, 2001). This reduction has been proposed to be important to transmit sustained

467 sensory stimulation (Kollmar et al., 1997). Perhaps local removal of $\mathrm{Ca}^{2+}$ into the mitochondria

468 during presynaptic activity is another mechanism in place to sustain neurotransmission. 
469 Alternatively, if mito- $\mathrm{Ca}^{2+}$ uptake does not buffer $\mathrm{Ca}^{2+}$, it could be critical to produce ATP for

470 other cellular tasks to maintain neurotransmission. Additional work is necessary to understand

471 how evoked mito-Ca ${ }^{2+}$ uptake functions to sustain presynaptic $\mathrm{Ca}^{2+}$ influx in mature-zebrafish

472 hair cells.

473 In addition to innate cellular roles, in neurons and in hair cells, mito-Ca ${ }^{2+}$ loading is also

474 associated with pathological processes such as reactive oxygen species (ROS) production, cell

475 death and synapse loss (Cai and Tammineni, 2016; Court and Coleman, 2012; DiMauro and

476 Schon, 2008; Esterberg et al., 2013, 2014; Sheng and Cai, 2012). Interestingly, recent work has

477 demonstrated that noise-induced hearing loss is associated with measurable changes in ribbon

478 morphology and synapse number (Jensen et al., 2015; Kujawa and Liberman, 2009; Liberman et

479 al., 2015). Work studying this type of hearing loss has shown that auditory inner hair cells in the

480 high frequency region of the mouse cochlea have enlarged ribbons immediately after noise,

481 followed later by synapse loss (Liberman et al., 2015). This pathology is reminiscent of our 1-hr

482 pharmacological treatments that completely block the MCU in mature zebrafish hair cells

483 (Figure 3E-F). After this treatment, we observed a reduction in the number hair cells and

484 synapses, and an increase in ribbon size. Surprisingly, these same treatments applied to

485 developing hair cells increased ribbon size but did not reduce cell or synapse number (Figure

486 5D). Recent work has suggested that younger hair cells may be more resilient to ototoxins,

487 perhaps because they have not yet accumulated an excess of mitochondria oxidation (Pickett et

488 al., 2018). This could explain why complete MCU block is not pathological to developing hair

489 cells. Overall these studies, along with our own data indicate that in mature hair cells, the

490 mitochondria and the MCU may be associated with pathological processes associated with

491 ototoxins and noise-exposure.

492 In further support of this idea, recent work in mice has investigated the role of the MCU

493 in noise-related hearing loss (Wang et al., 2018). This work demonstrated that pharmacological

494 block or a loss of function mutation in MCU protected against synapse loss in auditory inner

495 hair cells after noise exposure. Although this result is counter to our observed results where

496 complete MCU block reduces synapse number (Figure 3E), it highlights an association between

497 mito- $\mathrm{Ca}^{2+}$, noise exposure and synapse integrity. It is possible that these differences can be 
498 explained by transitory versus chronic alterations in mito-Ca ${ }^{2+}$ homeostasis. These differences

499 may be resolved by studying the hair cells in a zebrafish MCU knock out. In the future it will be

500 interesting to examine both mito- $\mathrm{Ca}^{2+}$ uptake and ribbon morphology during other pathological

501 conditions that enlarge ribbons such as noise exposure, ototoxicity and aging.

503 Role of spontaneous mito- $\mathrm{Ca}^{2+}$ uptake in developing hair cells

$504 \quad$ Although mitochondria have been studied in the context of cellular function and cell

505 death, relatively few studies have examined the role mitochondria play in development. We

506 found that mitochondria spontaneously take up $\mathrm{Ca}^{2+}$ during hair-cell development (Figure 4B-

507 C). Although studies in mammalian hair cells have demonstrated that there are spontaneous

508 rises in presynaptic $\mathrm{Ca}^{2+}$ during development (Marcotti et al., 2003; Tritsch et al., 2010), these

$509 \mathrm{Ca}^{2+}$ signals have not been reported in zebrafish hair cells. Our work highlights the mitochondria

510 as a downstream signaling organelle that couples presynaptic-Ca ${ }^{2+}$ influx to ribbon formation

511 (Figure 8A). In the future, zebrafish will be a useful model to further explore the origin and role

512 of these spontaneous $\mathrm{Ca}^{2+}$ signals.

513 In our study, we also found that altering baseline mito-Ca ${ }^{2+}$ levels rapidly influenced the

$514 \mathrm{NAD}^{+} / \mathrm{NADH}$ ratio and altered ribbon size in developing hair cells (Figure 5, 6, 7). However, in

515 mature hair cells, while alterations to mito-Ca ${ }^{2+}$ levels increased ribbon size they did not

516 influence $N A D(H)$ redox (Figure 6I). One reason why $N A D(H)$ redox does not change in mature

517 hair cells is that ribbon enlargement could be occurring through a different mechanism. For

518 example, ribbon enlargement could be a pathological byproduct of synapse loss (Figure 3E). In

519 mature hair cells, after MCU block it is possible that individual ribbons are not enlarging, but

520 instead ribbons are merging together as synapses are lost. In the future live imaging studies will

521 help resolve whether there are different mechanisms underlying ribbon enlargement in mature

522 and developing hair cells.

523 Overall this study has demonstrated the zebrafish-lateral line is a valuable system to

524 study the interplay between the mitochondria, and synapse function, development and

525 integrity. In the future it will be exciting to expand this research to explore how evoked and 
526 spontaneous mito-Ca ${ }^{2+}$ influx are impacted by pathological treatments such as age, noise and

527 ototoxins.

528

\section{Method Details}

\section{Zebrafish husbandry and genetics}

Adult Danio rerio (zebrafish) were maintained under standard conditions. Larvae 2 to 6

533 days post-fertilization (dpf) were maintained in $\mathrm{E} 3$ embryo medium (in $\mathrm{mM}: 5 \mathrm{NaCl}, 0.17 \mathrm{KCl}$,

$5340.33 \mathrm{CaCl}_{2}$ and $0.33 \mathrm{MgSO}_{4}$, buffered in $\mathrm{HEPES} \mathrm{pH} 7.2$ ) at $28^{\circ} \mathrm{C}$. All husbandry and experiments

535 were approved by the NIH Animal Care and Use program under protocol \#1362-13. Transgenic

536 zebrafish lines used in this study include: $T g$ (myo6b:GCaMP6s-CAAX) ${ }^{i d c 1}$ (Jiang et al., 2017b),

537 Tg(myo6b:RGECO1) ${ }^{\text {vo10Tg }}$ (Maeda et al., 2014), Tg(myo6b:GCaMP3)w78Tg (Esterberg et al., 2013),

$538 T g$ (myo6b:mitoGCaMP3) ${ }^{\text {119Tg }}$ (Esterberg et al., 2014), and Tg(myo6b:ribeye a-tagRFP) ${ }^{\text {idc11Tg }}$

539 (Sheets, 2017). Experiments were performed using Tübingen or TL wildtype strains.

Cloning and Transgenic Fish Production

To create transgenic fish, plasmid construction was based on the tol2/Gateway zebrafish

543 kit developed by the lab of Chi-Bin Chien at the University of Utah (Kwan et al., 2007). These

544 methods were used to create $T g$ (myo6b:mitoRGECO1) idc12Tg and Tg(myo6b:Rex-YFP) idc13Tg

545 transgenic lines. Gateway cloning was used to clone Rex-YFP (Bilan et al., 2014) and

546 mitoRGECO1 into the middle entry vector pDONR221. For mitochondrial matrix targeting, the

547 sequence of cytochrome C oxidase subunit VIII (Rizzuto et al., 1989) was added to the N-

548 terminus of RGECO1. Vectors p3E-polyA (Kwan et al., 2007) and pDestTol2CG2 (Kwan et al.,

549 2007) were recombined with p5E myosinVIb (myo6b) (Kindt et al., 2012) and our engineered

550 plasmids to create the following constructs: myo6b:REX-YFP, and myo6b:mitoRGECO1. To

551 generate transgenic fish, DNA clones $(25-50 \mathrm{ng} / \mu \mathrm{l})$ were injected along with tol2 transposase

552 mRNA $(25-50 \mathrm{ng} / \mu \mathrm{l})$ into zebrafish embryos at the single-cell stage. 
For immunohistological studies, zebrafish larvae were exposed to compounds diluted in

556 E3 with 0.1\% DMSO (isradipine, Bay K8644, NAD+ (Sigma-Aldrich, St. Louis, MO), Ru360

557 (Millipore, Burlington, MA)) or Tris-HCl (NADH (Cayman Chemical, Ann Arbor, MI)) for 30 min or

$5581 \mathrm{hr}$ at the concentrations indicated. E3 with $0.1 \% \mathrm{DMSO}$ or Tris- $\mathrm{HCl}$ were used as control

559 solutions. In solution at $\mathrm{pH} 7.0-7.3, \mathrm{NADH}$ oxidizes into $\mathrm{NAD}^{+}$by exposure to dissolved oxygen.

560 To mitigate this, NADH was dissolved immediately before use, and was exchanged with a

561 freshly dissolved NADH solution every half hour. Dosages of isradipine, Ru360, NAD ${ }^{+}$and NADH

562 did not confer excessive hair-cell death or synapse loss unless stated. After exposure to the

563 compounds, larvae were quickly sedated on ice and transferred to fixative.

In vivo imaging of baseline $\mathrm{Ca}^{2+}$ and $\mathrm{NAD}(\mathrm{H})$ redox

To prepare larvae for imaging, larvae were immobilized as previously described (Kindt et al, 2012). Briefly, larvae were anesthetized with tricaine (0.03\%) and pinned to a chamber lined with Sylgard 184 Silicone Elastomer (Dow Corning, Midland, MI). Larvae were injected with 125 $\mu \mathrm{M} \alpha$-bungarotoxin (Tocris, Bristol, UK) into the pericardial cavity to paralyze. Tricaine was rinsed off the larvae with E3.

For baseline measurements of Rex-YFP and cytoRGECO1 fluorescence, larvae were

572 imaged using an upright Nikon ECLIPSE Ni-E motorized microscope (Nikon Inc., Tokyo, Japan) in

573 widefield mode with a Nikon 60x 1.0 NA CFI Fluor water-immersion objective, 480/30 nm

574 excitation and $535 / 40 \mathrm{~nm}$ emission filter set or 520/35 $\mathrm{nm}$ excitation and 593/40 emission filter

575 set, and an ORCA-D2 camera (Hamamatsu Photonics K.K., Hamamatsu City, Japan). Acquisitions

576 were taken at $5 \mathrm{~Hz}$, in 15 plane Z-stacks every $2 \mu \mathrm{m}$. For baseline measurements of

577 MitoGCaMP3, larvae were imaged using a Bruker Swept-field confocal microscope (Bruker Inc.,

578 Billerica, MA), with a Nikon CFI Fluor 60x 1.0 NA water immersion objective. A Rolera EM-C2

579 CCD camera (QImaging, Surrey, Canada) was used to detect signals. Acquisitions were taken

580 using a $70 \mu \mathrm{m}$ slit at a frame rate of $10 \mathrm{~Hz}$, in 26 plane Z-stacks every $1 \mu \mathrm{m}$. MitoGCaMP3

581 baseline intensity varied dramatically in controls between timepoints. To offset this variability,

582 we acquired and averaged the intensity of 4 Z-stacks per time point. For all baseline

583 measurements transgenic larvae were first imaged in $\mathrm{E3}$ with $0.1 \% \mathrm{DMSO}$ or $0.1 \% \mathrm{Tris}-\mathrm{HCl}$ as 
appropriate. Then larvae were exposed to pharmacological agents for 30 minutes and a second acquisition was taken. Any neuromasts with cell death after pharmacological treatment were excluded from our analyses.

In vivo imaging of evoked $\mathrm{Ca}^{2+}$ signals

To measure evoked $\mathrm{Ca}^{2+}$ signals in hair-cells, larvae were prepared in a similar manner as described for baseline measurements. After $\alpha$-bungarotoxin paralysis, larvae were immersed

591 in neuronal buffer solution (in $\mathrm{mM}: 140 \mathrm{NaCl}, 2 \mathrm{KCl}, 2 \mathrm{CaCl}_{2}, 1 \mathrm{MgCl}_{2}$ and $10 \mathrm{HEPES}, \mathrm{pH}$ 7.3).

592 Evoked $\mathrm{Ca}^{2+}$ measurements were acquired using the Bruker Swept-field confocal system

593 described above. To stimulate lateral-line hair cells, a fluid-jet was used as previously described 594 to deliver a saturating stimulus (Lukasz and Kindt, 2018).

595 To measure presynaptic GCaMP6sCAAX signals at ribbons, images were acquired with 1 $596 \times 1$ binning with a $35 \mu \mathrm{m}$ slit at $50 \mathrm{~Hz}$ in a single plane containing presynaptic ribbons. Ribbons 597 were marked in live hair cells using the $T g$ (myo6b:ribeye a-tagRFP) ${ }^{i d c 11 T g}$ transgenic line (Figure 598 S2). Ribbons were located relative to GCaMP6s signals by acquiring a Z-stack of 5 planes $1 \mu \mathrm{m}$. 599 To correlate presynaptic GCaMP6sCAAX signals with mitoRGECO1 signals in hair cells, 2-color 600 imaging was performed. Images were acquired in a single plane with $2 \times 2$ binning at $10 \mathrm{~Hz}$. 601 MitoGCaMP3 signals were acquired in Z-stacks of 5 planes $1 \mu \mathrm{m}$ apart at $2 \times 2$ binning. High 602 speed imaging along the Z-axis was accomplished by using a piezoelectric motor (PICMA P$603882.11-888.11$ series, Physik Instrumente $\mathrm{GmbH}$, Karlsruhe, Germany) attached to the objective 604 to allow rapid imaging at a $50 \mathrm{~Hz}$ frame rate yielding a $10 \mathrm{~Hz}$ volume rate. For pharmacological 605 treatment, acquisitions were made prior to drug treatment and after a 20-min incubation in the 606 pharmacological agent. Any neuromasts with cell death after pharmacological treatment were 607 excluded from our analyses.

In vivo imaging of spontaneous $\mathrm{Ca}^{2+}$ signals

To measure spontaneous $\mathrm{Ca}^{2+}$ signals in hair-cells, larvae were prepared in a similar 611 manner as described for evoked $\mathrm{Ca}^{2+}$ measurements. Spontaneous $\mathrm{Ca}^{2+}$ measurements were 612 acquired using the Bruker Swept-field confocal system described above. To measure 
613 spontaneous presynaptic GCaMP6sCAAX signals, images were acquired with $2 \times 2$ binning with

614 a $70 \mu \mathrm{m}$ slit at $0.33 \mathrm{~Hz}$ in a single plane for $900 \mathrm{~s}$. For acquisition of two-color spontaneous

615 presynaptic GCaMP6sCAAX and mitoRGECO1 signals images were acquired with 2 × 2 binning

616 with a $70 \mu \mathrm{m}$ slit at $0.2 \mathrm{~Hz}$ in a single plane for $900 \mathrm{~s}$.

Electron microscopy

619 Larvae were prepared for electron microscopy as described previously (Sheets, 2017).

620 Transverse serial sections ( $\sim 0 \mathrm{~nm}$ thin sections) were used to section through neuromasts.

621 Samples were imaged on a JEOL JEM-2100 electron microscope (JEOL Inc., Tokyo, Japan). The

622 distance from the edge of a ribbon density to the edge of the nearest mitochondria was

623 measured ( $n=17$ ribbons). In $74 \%$ of ribbons, a mitochondrion could be clearly identified within

$6241 \mu \mathrm{m}$ of a ribbon in a single section (17 out of 21 ribbons). All distances and perimeters were

625 measured in FIJI (Schindelin et al., 2012).

Immunofluorescence staining and Airyscan imaging

Whole larvae were fixed with $4 \%$ paraformaldehyde in PBS at $4^{\circ} \mathrm{C}$ for $3.5-4 \mathrm{hr}$ as previously described (Zhang et al., 2018b). Fixative was washed out with $0.01 \%$ Tween in PBS

630 (PBST) in 4 washes, 5 min each. Larvae were then washed for 5 min with $\mathrm{H}_{2} \mathrm{O}$. The $\mathrm{H}_{2} \mathrm{O}$ was

631 thoroughly removed and replaced with ice-cold acetone and placed at $-20^{\circ} \mathrm{C}$ for $3 \mathrm{~min}$ for $3 \mathrm{dpf}$

632 and $5 \mathrm{~min}$ for $5 \mathrm{dpf}$ larvae, followed by a $5 \mathrm{~min}_{2} \mathrm{O}$ wash. The larvae were then washed for $4 \mathrm{x}$

$6335 \mathrm{~min}$ in PBST, then incubated in block overnight at $4^{\circ} \mathrm{C}$ in blocking solution (2\% goat serum, $1 \%$

634 bovine serum albumin, 2\% fish skin gelatin in PBST). Primary and secondary antibodies were

635 diluted in blocking solution. Primary antibodies and their respective dilution are: Ribbon label:

636 Mouse anti-Ribeye b IgG2a, 1:10,000 (Sheets et al., 2011b); PSD label: Mouse anti-pan-MAGUK

637 IgG1 \#75-029, 1:500 (UC Davis/NIH NeuroMab Facility, Davis, CA); Hair cell label: Rabbit anti-

638 Myosin VIIa, 1:1000 (Proteus BioSciences Inc., Ramona, CA). Larvae were incubated in primary

639 antibody solution for $2 \mathrm{hr}$ at room temperature. After $4 \times 5 \mathrm{~min}$ washes in PBST to remove the

640 primary antibodies, diluted secondary antibodies were added in and samples were incubated

641 for $2 \mathrm{hr}$ at room temperature. Secondary antibodies and their respective dilution are: goat anti- 
642 mouse IgG2a, Alexa Fluor 488, 1:1000; goat anti-rabbit IgG $(\mathrm{H}+\mathrm{L})$ Alexa Fluor 568, 1:1000; goat

643 anti-mouse IgG1 Alexa Fluor 647, 1:1000 (Thermo Fisher Scientific, Waltham, MA). Secondary

644 antibody was washed out with PBST for $3 \times 5$ min, followed by a 5 min wash with $\mathrm{H}_{2} \mathrm{O}$. Larvae

645 were mounted on glass slides with Prolong Gold Antifade Reagent (Invitrogen, Carlsbad, CA)

646 using No. 1.5 coverslips.

647 Prior to Airyscan imaging, live samples were immobilized in $2 \%$ low-melt agarose in 648 tricaine $(0.03 \%)$ in cover-glass bottomed dishes. Live and fixed samples were imaged on an 649 inverted Zeiss LSM 780 laser-scanning confocal microscope with an Airyscan attachment (Carl 650 Zeiss AG, Oberkochen, Germany) using an 63x 1.4 NA oil objective lens. The median ( \pm median 651 absolute deviation) lateral and axial resolution of the system was measured at $198 \pm 7.5 \mathrm{~nm}$ 652 and $913 \pm 50 \mathrm{~nm}$ (full-width at half-maximum), respectively. The acquisition parameters were 653 adjusted using the control sample such that pixels for each channel reach at least $1 / 10$ of the 654 dynamic range. The Airyscan Z-stacks were processed with Zeiss Zen Black software v2.1 using $6553 \mathrm{D}$ filter setting of 7.0. Experiments were imaged with the same acquisition settings to maintain consistency between comparisons.

\section{Quantification and Statistical Analysis}

659 Analysis of $\mathrm{Ca}^{2+}$ and $\mathrm{NAD}(H)$ signals, processing, and quantification

To quantify changes in baseline $\mathrm{Ca}^{2+}$ and $\mathrm{NAD}(\mathrm{H})$ homeostasis, images were processed in

661 FIJI. For our measurements we quantified the fluorescence in the basal-most $8 \mu \mathrm{m}$ (4 planes) to 662 avoid overlap between cells. The basal planes were max Z-projected, and a 24.0 $\mu \mathrm{m}$ (Rex-YFP 663 and RGECO1) or $26.8 \mu \mathrm{m}$ (MitoGCaMP3) circular region of interest (ROI) was drawn over the 664 neuromast to make an intensity measurement. To correct for photobleaching, a set of mock665 treated control neuromasts were imaged during every trial. These mock treatments were used 666 to normalize the post-treatment intensity values.

668 processed in FIJI. Images in each time series were aligned using Stackreg (Thevenaz et al., 669 1998). For evoked MitoRGECO1, MitoGCaMP3, CytoGCaMP3 and two-color GCaMP6sCAAX and

670 MitoRGECO1 signals, Z-stack were max z-projected, and a $5 \mu \mathrm{m}$ diameter circular ROI was 
671 drawn over each hair cell. For ribbon-localized measurements, GCaMP6sCAAX signals were

672 measured within a $1.34 \mu \mathrm{m}$ round ROls at individual ribbons, and intensity of multiple ROI

673 within a cell were averaged. Cells with presynaptic $\mathrm{Ca}^{2+}$ activity is defined by max $\Delta \mathrm{F} / \mathrm{F}$ of $>0.05$

674 for MitoRGECO1 and MitoGCaMP3, and $\max \triangle \mathrm{F} / \mathrm{F}>0.25$ for GCaMP6sCAAX for a 2-s

675 stimulation.

676 To quantify the average magnitude and frequency of spontaneous $\mathrm{Ca}^{2+}$ changes in

677 GCaMP6sCAAX signals, images were processed in Matlab R2014b (Mathworks, Natick, MA) and

678 FIJI. First, images in each time series were aligned using Stackreg (Thevenaz et al., 1998). To

679 measure the average magnitude during the $900 \mathrm{~s}$ GCaMP6sCAAX image acquisition, a $5 \mu \mathrm{m}$

680 diameter circular ROI was drawn over each hair cell and a raw intensity value was obtained

681 from each time point. Then the raw traces were bleach corrected. Next, the corrected intensity

682 values were normalized as $\Delta \mathrm{F} / \mathrm{F}_{\mathrm{o}} . \mathrm{F}_{\mathrm{o}}$ is defined as the bottom $15^{\text {th }}$ percentile of fluorescence

683 values (Babola et al., 2018). Then, values of $\Delta F / F_{0}$ of less than $10 \%$ were removed. These values

684 were considered to be noise and our threshold value for a true signal. A $10 \%$ threshold was

685 determined by imaging spontaneous GCaMP6CAAX signals in the presence of isradipine where

686 no signals were observed (Figure S4). The averaged magnitude of spontaneous activity per cell

687 was obtained by dividing the integral/sum of GCaMP6sCAAX signals $\left(\triangle F / F_{0}>10 \%\right)$ during the

688 whole recording period by 300 (300 frames in 900 s). The frequency of GCaMP6sCAAX signals

689 was defined as the average number of peaks per second during the whole recording period.

691 Image processing and quantification of synapse morphology

To quantify synapse morphology and pairing, images were first processed in ImageJ

693 (NIH, Bethesda, MD), and then synapses were paired using Python (Python Software

694 Foundation, Wilmington, DE) in the Spyder Scientific Environment (MIT, Cambridge, MA). In

695 ImageJ, each Airyscan Z-stack was background subtracted using rolling-ball subtraction. Z-stacks

696 containing the MAGUK channel were further bandpass filtered to remove details smaller than 6

697 px and larger than 20 px. A duplicate of the Z-stack was normalized for intensity. This duplicated

698 Z-stack was used to identify individual ribbon and MAGUK using the Simple 3D Segmentation of

699 ImageJ 3D Suite (Ollion et al., 2013). Local intensity maxima, identified with 3D Fast Filter, and 
700 3D watershed were used to separate close-by structures. The centroid for each identified

701 ribbon and MAGUK was obtained using 3D Manager and were used to identify complete

702 synapses. The max Z-projection of the segmented Z-stack was used to generate a list of 2D

703 objects as individual ROIs corresponding to each punctum. This step also included a minimum

704 size filter, Ribeye: $0.08 \mu \mathrm{m}^{2}$, MAGUK $0.04 \mu \mathrm{m}^{2}$. For quantification of extrasynaptic Ribeye $\mathrm{b}$

705 puncta, the minimum size filter was not applied. The 2D puncta ROI were applied over the max

706 Z-projection of the original Z-stack processed only with background subtraction. This step

707 measures the intensity of the antibody label. Centroid and intensity information were exported

708 as a CSV spreadsheet (macro is available upon request).

709 In Python, the 3D centroid coordinates for each ribbon punctum was measured against

710 the coordinates of every post-synaptic MAGUK punctum to find the MAGUK punctum within a

711 threshold distance. This threshold was calculated by taking the 2D area of the Ribeye and

712 MAGUK punctum measured in the max Z-projection to calculate an approximate radius by

713 dividing by $\pi$ and taking the square root. The two radii were then summed to get the threshold.

714 Puncta that were not paired were excluded from later statistical analyses of synaptic ribbon and

715 postsynaptic MAGUK puncta. Hair cell and synapse count were confirmed manually. Hair cell

716 counts were performed with myosin VIla antibody label in treatments where synapse or cell

717 numbers were reduced.

\section{Statistics}

720 Statistical analyses and data plots were performed with Prism 8 (Graphpad, San Diego,

721 CA). Values in the text and data with error bars on graphs and in text are expressed as mean \pm

722 SEM unless indicated otherwise. All experiments were performed on a minimum of 2 animals, 6

723 neuromasts (posterior lateral-line neuromasts L1-L4), on 2 independent days. For 3 and 5 dpf

724 larvae each neuromast represents analysis from 8-12 hair cells; 24-36 synapses and 14-18 hair

725 cells; 42-54 synapses respectively. All replicates are biological. Based on the variance and effect

726 sizes reported previously and measured in this study, these numbers were adequate to provide

727 statistical power to avoid both Type I and Type II error (Sheets et al., 2012; Zhang et al., 2018b).

728 No animals or samples were excluded from our analyses unless control experiments failed-in 
these cases all samples were excluded. No randomization or blinding was used for our animal studies. Where appropriate, datasets were confirmed for normality using a D'Agostino-Pearson normality test and for equal variances using a F test to compare variances. Statistical

732 significance between two conditions was determined by either unpaired Welch's unequal

733 variance $t$-test, a Mann-Whitney $U$ test or a Wilcoxon matched-pairs signed-rank test as

734 appropriate. For comparison of multiple conditions, a Brown-Forsythe or a Welch ANOVA with

735 Games-Howell post hoc were used.

\section{Acknowledgements}

738 This work was supported by National Institute on Deafness and Other Communication Disorders Intramural Research Program Grant 1ZIADC000085-01 to K.S.K. and ZICDC000081 to R.S.P. and Y.-X.W. We would like to thank Katie Drerup, Paul Fuchs and Doris Wu for their support and thoughtful comments on the manuscript.

\section{Declaration of interests}

744 The authors declare no competing financial or non-financial interests.

\section{Author contributions}

747 Conceptualization, Methodology, Writing, H.C.W., K.S.K., Formal Analysis, H.C.W., Investigation, 748 H.C.W., Q.X.Z., A.J.B., R.S.P., Y.-X.W., and Supervision, K.S.K.

\section{References}

751 Babola, T.A., Li, S., Gribizis, A., Lee, B.J., Issa, J.B., Wang, H.C., Crair, M.C., and Bergles, D.E. 752 (2018). Homeostatic Control of Spontaneous Activity in the Developing Auditory System.

753 Neuron 99, 511-524.e5.

754 Becker, L., Schnee, M.E., Niwa, M., Sun, W., Maxeiner, S., Talaei, S., Kachar, B., Rutherford, 755 M.A., and Ricci, A.J. (2018). The presynaptic ribbon maintains vesicle populations at the hair cell 756 afferent fiber synapse. ELife Sciences 7, e30241.

757 Billups, B., and Forsythe, I.D. (2002). Presynaptic mitochondrial calcium sequestration 758 influences transmission at mammalian central synapses. The Journal of Neuroscience : The 759 Official Journal of the Society for Neuroscience 22, 5840-5847. 
760

761

762

763

764

765

766

767

768

769

770

771

772

773

774

775

776

777

778

779

780

781

782

783

784

785

786

787

788

789

790

791

792

Böttger, E.C., and Schacht, J. (2013). The mitochondrion: a perpetrator of acquired hearing loss. Hear. Res. 303, 12-19.

Brandt, A., Striessnig, J., and Moser, T. (2003). CaV1.3 channels are essential for development and presynaptic activity of cochlear inner hair cells. J. Neurosci. 23, 10832-10840.

Cai, Q., and Tammineni, P. (2016). Alterations in Mitochondrial Quality Control in Alzheimer's Disease. Front. Cell. Neurosci. 10.

Carafoli, E. (2011). The plasma membrane calcium pump in the hearing process: physiology and pathology. Sci China Life Sci 54, 686-690.

Castellano-Muñoz, M., and Ricci, A.J. (2014). Role of intracellular calcium stores in hair-cell ribbon synapse. Front Cell Neurosci 8.

Ceriani, F., Hendry, A., Jeng, J.-Y., Johnson, S.L., Stephani, F., Olt, J., Holley, M.C., Mammano, F., Engel, J., Kros, C.J., et al. (2019). Coordinated calcium signalling in cochlear sensory and nonsensory cells refines afferent innervation of outer hair cells. The EMBO Journal e99839.

Chinnadurai, G. (2007). Transcriptional regulation by C-terminal binding proteins. The International Journal of Biochemistry \& Cell Biology 39, 1593-1607.

Chouhan, A.K., Zhang, J., Zinsmaier, K.E., and Macleod, G.T. (2010). Presynaptic mitochondria in functionally different motor neurons exhibit similar affinities for $\mathrm{Ca} 2+$ but exert little influence as $\mathrm{Ca} 2+$ buffers at nerve firing rates in situ. The Journal of Neuroscience : The Official Journal of the Society for Neuroscience 30, 1869-1881.

Costalupes, J.A., Young, E.D., and Gibson, D.J. (1984). Effects of continuous noise backgrounds on rate response of auditory nerve fibers in cat. Journal of Neurophysiology 51, 1326-1344.

Court, F.A., and Coleman, M.P. (2012). Mitochondria as a central sensor for axonal degenerative stimuli. Trends Neurosci. 35, 364-372.

Devine, M.J., and Kittler, J.T. (2018). Mitochondria at the neuronal presynapse in health and disease. Nature Reviews Neuroscience 19, 63-80.

DiMauro, S., and Schon, E.A. (2008). Mitochondrial Disorders in the Nervous System. Annual Review of Neuroscience 31, 91-123.

Eckrich, T., Blum, K., Milenkovic, I., and Engel, J. (2018). Fast Ca2+ Transients of Inner Hair Cells Arise Coupled and Uncoupled to Ca2+ Waves of Inner Supporting Cells in the Developing Mouse Cochlea. Front. Mol. Neurosci. 11.

Esterberg, R., Hailey, D.W., Coffin, A.B., Raible, D.W., and Rubel, E.W. (2013). Disruption of Intracellular Calcium Regulation Is Integral to Aminoglycoside-Induced Hair Cell Death. Journal of Neuroscience 33, 7513-7525. 

Flow Underlies Vulnerability of Mechanosensory Hair Cells to Damage. J Neurosci 34, 97039719.

Fischel-Ghodsian, N., Kopke, R.D., and Ge, X. (2004). Mitochondrial dysfunction in hearing loss. Mitochondrion 4, 675-694. allows the transcriptional corepressor carboxyl-terminal binding protein to serve as a metabolic sensor. Proc. Natl. Acad. Sci. U.S.A. 100, 9202-9207.

801 Flippo, K.H., and Strack, S. (2017). Mitochondrial dynamics in neuronal injury, development and plasticity. Journal of Cell Science 130, 671-681.

803 Furman, A.C., Kujawa, S.G., and Liberman, M.C. (2013). Noise-induced cochlear neuropathy is 804 selective for fibers with low spontaneous rates. J. Neurophysiol. 110, 577-586.

805 Hübler, D., Rankovic, M., Richter, K., Lazarevic, V., Altrock, W.D., Fischer, K.-D., Gundelfinger, 806 E.D., and Fejtova, A. (2012). Differential spatial expression and subcellular localization of CtBP 807 family members in rodent brain. PloS One 7, e39710.

808 Ivanova, D., Dirks, A., Montenegro-Venegas, C., Schöne, C., Altrock, W.D., Marini, C., 809 Frischknecht, R., Schanze, D., Zenker, M., Gundelfinger, E.D., et al. (2015). Synaptic activity 810 controls localization and function of CtBP1 via binding to Bassoon and Piccolo. EMBO J 34, 811 1056-1077.

812 Jean, P., Morena, D.L. de la, Michanski, S., Tobón, L.M.J., Chakrabarti, R., Picher, M.M., Neef, J., 813 Jung, S., Gültas, M., Maxeiner, S., et al. (2018). The synaptic ribbon is critical for sound encoding 814 at high rates and with temporal precision. ELife Sciences 7, e29275.

815 Jensen, J.B., Lysaght, A.C., Liberman, M.C., Qvortrup, K., and Stankovic, K.M. (2015). Immediate 816 and delayed cochlear neuropathy after noise exposure in pubescent mice. PloS One 10, 817 e0125160.

818 Jensen-Smith, H.C., Hallworth, R., and Nichols, M.G. (2012). Gentamicin rapidly inhibits 819 mitochondrial metabolism in high-frequency cochlear outer hair cells. PLoS ONE 7, e38471.

820 Jiang, T., Kindt, K., and Wu, D.K. (2017a). Transcription factor Emx2 controls stereociliary bundle 821 orientation of sensory hair cells. ELife 6.

822 Jiang, T., Kindt, K., and Wu, D.K. (2017b). Transcription factor Emx2 controls stereociliary bundle 823 orientation of sensory hair cells. ELife 6.

824 Johnson, S.L., Safieddine, S., Mustapha, M., and Marcotti, W. (2019). Hair Cell Afferent 825 Synapses: Function and Dysfunction. Cold Spring Harb Perspect Med. 
826 Kalluri, R., and Monges-Hernandez, M. (2017). Spatial Gradients in the Size of Inner Hair Cell 827 Ribbons Emerge Before the Onset of Hearing in Rats. Journal of the Association for Research in 828 Otolaryngology 18, 399-413.

829 Kann, O., and Kovács, R. (2007). Mitochondria and neuronal activity. Am. J. Physiol., Cell Physiol. $830 \quad 292$, C641-57.

831 Kennedy, H.J. (2002). Intracellular calcium regulation in inner hair cells from neonatal mice. Cell 832 Calcium 31, 127-136.

833 Kindt, K.S., Finch, G., and Nicolson, T. (2012). Kinocilia mediate mechanosensitivity in 834 developing zebrafish hair cells. Developmental Cell 23, 329-341.

835 Kokotas, H., Petersen, M.B., and Willems, P.J. (2007). Mitochondrial deafness. Clin. Genet. 71, 836 379-391.

837 Kollmar, R., Fak, J., Montgomery, L.G., and Hudspeth, A.J. (1997). Hair cell-specific splicing of 838 mRNA for the alpha1D subunit of voltage-gated Ca2+ channels in the chicken's cochlea. Proc. 839 Natl. Acad. Sci. U.S.A. 94, 14889-14893.

840 Koschak, A., Reimer, D., Huber, I., Grabner, M., Glossmann, H., Engel, J., and Striessnig, J. 841 (2001). alpha 1D (Cav1.3) subunits can form l-type Ca2+ channels activating at negative 842 voltages. J. Biol. Chem. 276, 22100-22106.

843 Kujawa, S.G., and Liberman, M.C. (2009). Adding insult to injury: cochlear nerve degeneration 844 after "temporary" noise-induced hearing loss. The Journal of Neuroscience : The Official Journal 845 of the Society for Neuroscience 29, 14077-14085.

846 Kwan, K.M., Fujimoto, E., Grabher, C., Mangum, B.D., Hardy, M.E., Campbell, D.S., Parant, J.M., 847 Yost, H.J., Kanki, J.P., and Chien, C.-B. (2007). The Tol2kit: a multisite gateway-based 848 construction kit for Tol2 transposon transgenesis constructs. Dev. Dyn. 236, 3088-3099.

849 Kwon, S.-K., Sando, R., Lewis, T.L., Hirabayashi, Y., Maximov, A., and Polleux, F. (2016). LKB1

850 Regulates Mitochondria-Dependent Presynaptic Calcium Clearance and Neurotransmitter 851 Release Properties at Excitatory Synapses along Cortical Axons. PLOS Biology 14, e1002516.

852 Lagnado, L., and Schmitz, F. (2015). Ribbon Synapses and Visual Processing in the Retina.

853 Annual Review of Vision Science 1, 235-262.

854 Lepeta, K., Lourenco, M.V., Schweitzer, B.C., Martino Adami, P.V., Banerjee, P., Catuara-Solarz, 855 S., de La Fuente Revenga, M., Guillem, A.M., Haidar, M., ljomone, O.M., et al. (2016).

856 Synaptopathies: synaptic dysfunction in neurological disorders - A review from students to 857 students. J Neurochem 138, 785-805.

858 Levy, M., Faas, G.C., Saggau, P., Craigen, W.J., and Sweatt, J.D. (2003). Mitochondrial Regulation 859 of Synaptic Plasticity in the Hippocampus. Journal of Biological Chemistry 278, 17727-17734. 
860 Liberman, L.D., and Liberman, M.C. (2016). Postnatal maturation of auditory-nerve

861 heterogeneity, as seen in spatial gradients of synapse morphology in the inner hair cell area.

862 Hearing Research 339, 12-22.

863 Liberman, L.D., Wang, H., and Liberman, M.C. (2011). Opposing Gradients of Ribbon Size and 864 AMPA Receptor Expression Underlie Sensitivity Differences among Cochlear-Nerve/Hair-Cell

865 Synapses. J Neurosci 31, 801-808.

866 Liberman, L.D., Liberman, M.C., and Liberman, M.C. (2015). Dynamics of cochlear synaptopathy 867 after acoustic overexposure. Journal of the Association for Research in Otolaryngology 16, 205868219.

869 Liberman, M.C., Dodds, L.W., and Pierce, S. (1990). Afferent and efferent innervation of the cat 870 cochlea: quantitative analysis with light and electron microscopy. The Journal of Comparative 871 Neurology 301, 443-60.

872 Lioudyno, M., Hiel, H., Kong, J.-H., Katz, E., Waldman, E., Parameshwaran-lyer, S., Glowatzki, E., 873 and Fuchs, P.A. (2004). A "synaptoplasmic cistern" mediates rapid inhibition of cochlear hair 874 cells. J. Neurosci. 24, 11160-11164.

875 Llorente-Folch, I., Rueda, C.B., Pardo, B., Szabadkai, G., Duchen, M.R., and Satrustegui, J. (2015). 876 The regulation of neuronal mitochondrial metabolism by calcium. J Physiol 593, 3447-3462.

877 Lukasz, D., and Kindt, K.S. (2018). In Vivo Calcium Imaging of Lateral-line Hair Cells in Larval 878 Zebrafish. J Vis Exp.

879 Maeda, R., Kindt, K.S., Mo, W., Morgan, C.P., Erickson, T., Zhao, H., Clemens-Grisham, R., Barr880 Gillespie, P.G., and Nicolson, T. (2014). Tip-link protein protocadherin 15 interacts with 881 transmembrane channel-like proteins TMC1 and TMC2. Proceedings of the National Academy 882 of Sciences 111, 12907-12912.

883 Magupalli, V.G., Schwarz, K., Alpadi, K., Natarajan, S., Seigel, G.M., and Schmitz, F. (2008). 884 Multiple RIBEYE-RIBEYE interactions create a dynamic scaffold for the formation of synaptic 885 ribbons. The Journal of Neuroscience : The Official Journal of the Society for Neuroscience 28, $8867954-7967$.

887 Marcotti, W., Johnson, S.L., Rusch, A., and Kros, C.J. (2003). Sodium and calcium currents shape 888 action potentials in immature mouse inner hair cells. J. Physiol. (Lond.) 552, 743-761.

Matlib, M.A., Zhou, Z., Knight, S., Ahmed, S., Choi, K.M., Krause-Bauer, J., Phillips, R., Altschuld, 890 R., Katsube, Y., Sperelakis, N., et al. (1998). Oxygen-bridged dinuclear ruthenium amine complex 891 specifically inhibits Ca2+ uptake into mitochondria in vitro and in situ in single cardiac 892 myocytes. Journal of Biological Chemistry 273, 10223-10231.

893 Matthews, G., and Fuchs, P. (2010). The diverse roles of ribbon synapses in sensory neurotransmission. Nat. Rev. Neurosci. 11, 812-822. 
895 McHenry, M.J., Feitl, K.E., Strother, J.A., and Van Trump, W.J. (2009). Larval zebrafish rapidly sense the water flow of a predator's strike. Biology Letters 5, 477-479.

Merchan-Perez, A., and Liberman, M.C. (1996). Ultrastructural differences among afferent synapses on cochlear hair cells: correlations with spontaneous discharge rate. J. Comp. Neurol. $371,208-221$.

Metcalfe, W.K. (1985). Sensory neuron growth cones comigrate with posterior lateral line primordial cells in zebrafish. The Journal of Comparative Neurology 238, 218-224.

Moser, T., Brandt, A., and Lysakowski, A. (2006). Hair cell ribbon synapses. Cell Tissue Res 326, 347-359.

Mulkey, R.M., and Malenka, R.C. (1992). Mechanisms underlying induction of homosynaptic long-term depression in area CA1 of the hippocampus. Neuron 9, 967-975.

Murakami, S.L., Cunningham, L.L., Werner, L.A., Bauer, E., Pujol, R., Raible, D.W., and Rubel, E.W. (2003). Developmental differences in susceptibility to neomycin-induced hair cell death in the lateral line neuromasts of zebrafish (Danio rerio). Hearing Research 186, 47-56.

Ohn, T.-L., Rutherford, M.A., Jing, Z., Jung, S., Duque-Afonso, C.J., Hoch, G., Picher, M.M., Scharinger, A., Strenzke, N., and Moser, T. (2016). Hair cells use active zones with different voltage dependence of $\mathrm{Ca} 2+$ influx to decompose sounds into complementary neural codes. Proceedings of the National Academy of Sciences 113, E4716-E4725.

Ollion, J., Cochennec, J., Loll, F., Escudé, C., and Boudier, T. (2013). TANGO: a generic tool for high-throughput 3D image analysis for studying nuclear organization. Bioinformatics 29, 1840-

9151841.

916 Pickett, S.B., Thomas, E.D., Sebe, J.Y., Linbo, T., Esterberg, R., Hailey, D.W., and Raible, D.W. 917 (2018). Cumulative mitochondrial activity correlates with ototoxin susceptibility in zebrafish 918 mechanosensory hair cells. Elife 7.

919 Platzer, J., Engel, J., Schrott-Fischer, A., Stephan, K., Bova, S., Chen, H., Zheng, H., and Striessnig, 920 J. (2000). Congenital deafness and sinoatrial node dysfunction in mice lacking class D L-type 921 Ca2+ channels. Cell 102, 89-97.

922 Rizzuto, R., Nakase, H., Darras, B., Francke, U., Fabrizi, G.M., Mengel, T., Walsh, F., Kadenbach, 923 B., DiMauro, S., and Schon, E.A. (1989). A gene specifying subunit VIII of human cytochrome c 924 oxidase is localized to chromosome 11 and is expressed in both muscle and non-muscle tissues. 925 J. Biol. Chem. 264, 10595-10600.

926 Safieddine, S., El-Amraoui, A., and Petit, C. (2012). The Auditory Hair Cell Ribbon Synapse: From 927 Assembly to Function. Annual Review of Neuroscience 35, 509-528. 
928 Santos, F., MacDonald, G., Rubel, E.W., and Raible, D.W. (2006). Lateral line hair cell maturation 929 is a determinant of aminoglycoside susceptibility in zebrafish (Danio rerio). Hearing Research

$930213,25-33$.

931 Schindelin, J., Arganda-Carreras, I., Frise, E., Kaynig, V., Longair, M., Pietzsch, T., Preibisch, S., 932 Rueden, C., Saalfeld, S., Schmid, B., et al. (2012). Fiji: an open-source platform for biological-

933 image analysis. Nat. Methods 9, 676-682.

934 Schmitz, F., Königstorfer, A., and Südhof, T.C. (2000a). RIBEYE, a component of synaptic ribbons: 935 a protein's journey through evolution provides insight into synaptic ribbon function. Neuron 28, 936 857-872.

937 Schmitz, F., Königstorfer, A., and Südhof, T.C. (2000b). RIBEYE, a component of synaptic 938 ribbons: a protein's journey through evolution provides insight into synaptic ribbon function.

939 Neuron 28, 857-872.

940 Schnee, M.E., and Ricci, A.J. (2003). Biophysical and pharmacological characterization of 941 voltage-gated calcium currents in turtle auditory hair cells. J. Physiol. (Lond.) 549, 697-717.

942 Sheets, L. (2017). Excessive activation of ionotropic glutamate receptors induces apoptotic hair943 cell death independent of afferent and efferent innervation. Sci Rep 7, 41102.

944 Sheets, L., Trapani, J.G., Mo, W., Obholzer, N., and Nicolson, T. (2011a). Ribeye is required for 945 presynaptic $\mathrm{Ca}(\mathrm{V}) 1.3$ a channel localization and afferent innervation of sensory hair cells.

946 Development 138, 1309-1319.

947 Sheets, L., Trapani, J.G., Mo, W., Obholzer, N., and Nicolson, T. (2011b). Ribeye is required for 948 presynaptic $\mathrm{Ca}(\mathrm{V}) 1.3$ a channel localization and afferent innervation of sensory hair cells.

949 Development (Cambridge, England) 138, 1309-19.

950 Sheets, L., Kindt, K.S., and Nicolson, T. (2012). Presynaptic CaV1.3 channels regulate synaptic 951 ribbon size and are required for synaptic maintenance in sensory hair cells. J. Neurosci. 32, $952 \quad 17273-17286$.

953 Sheets, L., Hagen, M.W., and Nicolson, T. (2014). Characterization of Ribeye Subunits in 954 Zebrafish Hair Cells Reveals That Exogenous Ribeye B-Domain and CtBP1 Localize to the Basal 955 Ends of Synaptic Ribbons. PLOS ONE 9, e107256.

956 Sheets, L., He, X.J., Olt, J., Schreck, M., Petralia, R.S., Wang, Y.-X., Zhang, Q., Beirl, A., Nicolson, 957 T., Marcotti, W., et al. (2017). Enlargement of Ribbons in Zebrafish Hair Cells Increases Calcium 958 Currents But Disrupts Afferent Spontaneous Activity and Timing of Stimulus Onset. J. Neurosci. $95937,6299-6313$.

960 Sheng, Z.-H., and Cai, Q. (2012). Mitochondrial transport in neurons: impact on synaptic 961 homeostasis and neurodegeneration. Nature Reviews Neuroscience 13, 77-93. 
Sidi, S., Busch-Nentwich, E., Friedrich, R., Schoenberger, U., and Nicolson, T. (2004). gemini encodes a zebrafish L-type calcium channel that localizes at sensory hair cell ribbon synapses. J. Neurosci. 24, 4213-4223.

Song, H., Nie, L., Rodriguez-Contreras, A., Sheng, Z.-H., and Yamoah, E.N. (2003). Functional interaction of auxiliary subunits and synaptic proteins with $\mathrm{Ca}(\mathrm{v}) 1.3$ may impart hair cell $\mathrm{Ca} 2+$ current properties. J. Neurophysiol. 89, 1143-1149.

968 Song, Q., Shen, P., Li, X., Shi, L., Liu, L., Wang, J., Yu, Z., Stephen, K., Aiken, S., Yin, S., et al. 969 (2016). Coding deficits in hidden hearing loss induced by noise: the nature and impacts.

970 Scientific Reports 6, 25200.

971 Srivastava, S. (2016). Emerging therapeutic roles for NAD+ metabolism in mitochondrial and 972 age-related disorders. Clin Trans Med 5, 25.

973 Stankiewicz, T.R., Gray, J.J., Winter, A.N., and Linseman, D.A. (2014). C-terminal binding 974 proteins: central players in development and disease. Biomolecular Concepts 5, 489-511.

975 Thevenaz, P., Ruttimann, U.E., and Unser, M. (1998). A pyramid approach to subpixel 976 registration based on intensity. IEEE Transactions on Image Processing 7, 27-41.

977 Thio, S.S.C., Bonventre, J.V., and Hsu, S.I.-H. (2004). The CtBP2 co-repressor is regulated by 978 NADH-dependent dimerization and possesses a novel $\mathrm{N}$-terminal repression domain. Nucleic 979 Acids Res 32, 1836-1847.

980 Todorova, V., and Blokland, A. (2017). Mitochondria and Synaptic Plasticity in the Mature and Aging Nervous System. Current Neuropharmacology 15, 166-173. tom Dieck, S., Altrock, W.D., Kessels, M.M., Qualmann, B., Regus, H., Brauner, D., Fejtová, A., Bracko, O., Gundelfinger, E.D., and Brandstätter, J.H. (2005). Molecular dissection of the photoreceptor ribbon synapse. The Journal of Cell Biology 168, 825-836.

985 Tritsch, N.X., Yi, E., Gale, J.E., Glowatzki, E., and Bergles, D.E. (2007). The origin of spontaneous 986 activity in the developing auditory system. Nature 450, 50-55.

987 Tritsch, N.X., Rodríguez-Contreras, A., Crins, T.T.H., Wang, H.C., Borst, J.G.G., and Bergles, D.E. 988 (2010). Calcium action potentials in hair cells pattern auditory neuron activity before hearing 989 onset. Nat. Neurosci. 13, 1050-1052.

990 Tucker, T., and Fettiplace, R. (1995). Confocal imaging of calcium microdomains and calcium 991 extrusion in turtle hair cells. Neuron 15, 1323-1335.

992 Vos, M., Lauwers, E., and Verstreken, P. (2010). Synaptic mitochondria in synaptic transmission 993 and organization of vesicle pools in health and disease. Front Synaptic Neurosci 2, 139. 
994 Wang, X., Zhu, Y., Long, H., Pan, S., Xiong, H., Fang, Q., Hill, K., Lai, R., Yuan, H., and Sha, S.-H. 995 (2018). Mitochondrial Calcium Transporters Mediate Sensitivity to Noise-Induced Losses of Hair 996 Cells and Cochlear Synapses. Front Mol Neurosci 11, 469.

997 Xu, W., and Lipscombe, D. (2001). Neuronal Ca(V)1.3alpha(1) L-type channels activate at 998 relatively hyperpolarized membrane potentials and are incompletely inhibited by

999 dihydropyridines. J. Neurosci. 21, 5944-5951.

1000 Yamoah, E.N., Lumpkin, E.A., Dumont, R.A., Smith, P.J., Hudspeth, A.J., and Gillespie, P.G. 1001 (1998). Plasma membrane Ca2+-ATPase extrudes Ca2+ from hair cell stereocilia. J. Neurosci. 18, 1002 610-624.

1003 Yin, Y., Liberman, L.D., Maison, S.F., and Liberman, M.C. (2014). Olivocochlear innervation 1004 maintains the normal modiolar-pillar and habenular-cuticular gradients in cochlear synaptic 1005 morphology. Journal of the Association for Research in Otolaryngology : JARO 15, 571-83.

1006 Zenisek, D., and Matthews, G. (2000). The role of mitochondria in presynaptic calcium handling 1007 at a ribbon synapse. Neuron 25, 229-237.

1008 Zhang, L., Engler, S., Koepcke, L., Steenken, F., and Köppl, C. (2018a). Concurrent gradients of 1009 ribbon volume and AMPA-receptor patch volume in cochlear afferent synapses on gerbil inner 1010 hair cells. Hearing Research 364, 81-89.

1011 Zhang, Q., Li, S., Wong, H.-T.C., He, X.J., Beirl, A., Petralia, R.S., Wang, Y.-X., and Kindt, K.S. 1012 (2018b). Synaptically silent sensory hair cells in zebrafish are recruited after damage. Nat 1013 Commun 9, 1388. 

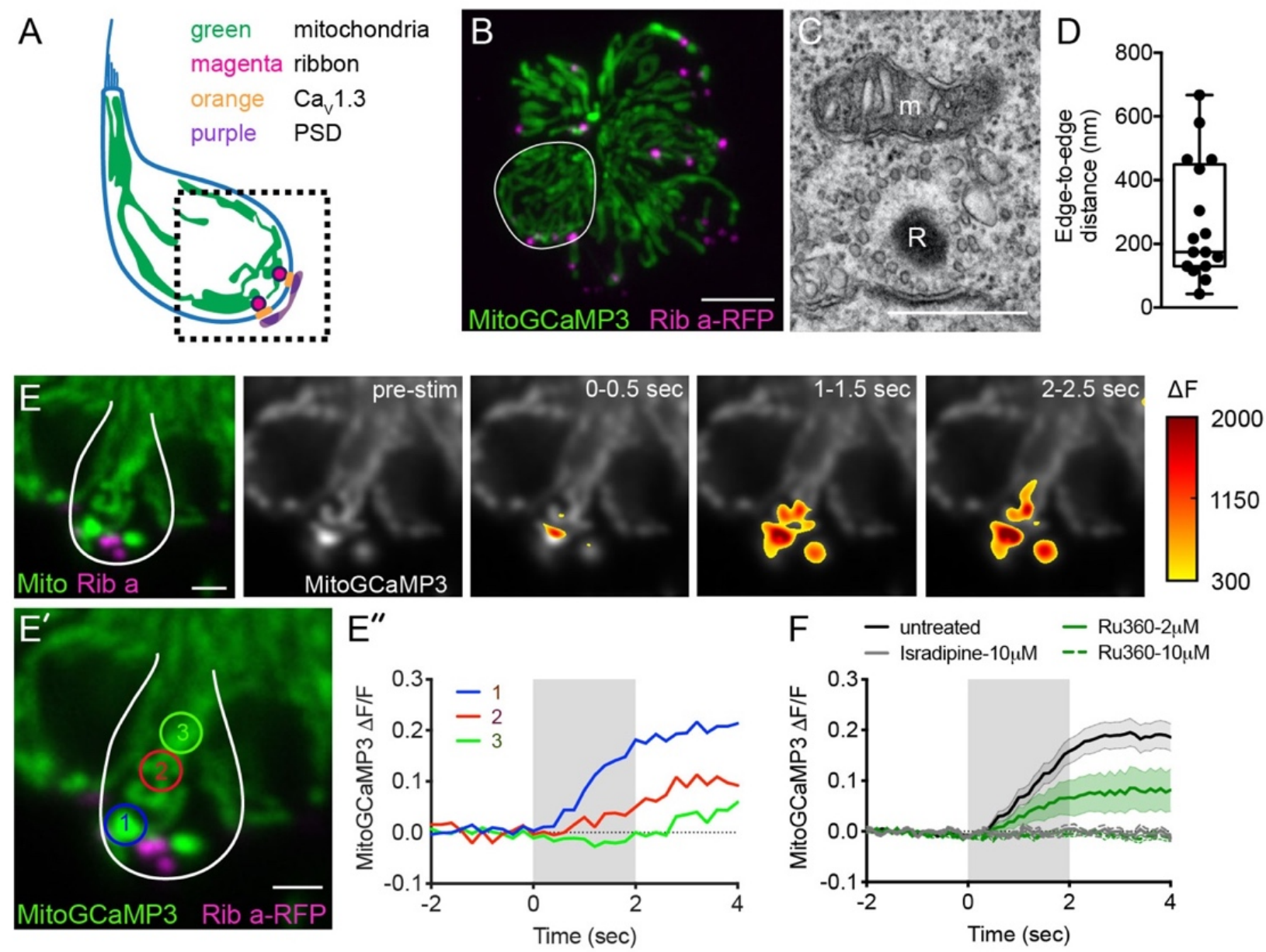

Figure 1. Mito- $\mathrm{Ca}^{2+}$ uptake initiates adjacent to ribbons. A, cartoon illustration of a lateral-line

1020 hair cell containing: an apical mechanosensory bundle (blue), mitochondria (green), presynaptic

1021 ribbons (magenta), Cav1.3 channels (orange) and postsynaptic densities (purple). B, Airyscan

1022 confocal image of 6 live hair cells (1 cell outlined in white) expressing MitoGCaMP3

1023 (mitochondria) and Ribeye a-tagRFP (ribbons) in a developing neuromast at 2 dpf. Also see

1024 Figure S1. C, A representative TEM showing a mitochondrion ( $\mathrm{m}$ ) in close proximity to a ribbon

1025 (R) at $4 \mathrm{dpf}$. D, Quantification of mitochondrion to ribbon distance in TEM sections $(n=17$

1026 sections). E, Side-view of a hair cell (outlined in white) shows the spatio-temporal dynamics of

1027 evoked mito-Ca ${ }^{2+}$ signals during a 2-s stimulation at $6 \mathrm{dpf}$. The MitoGCaMP3 signals are

1028 indicated by the heatmap and are overlaid onto the pre-stimulus grayscale image. E'-E', Circles

1029 1-3 (1.3 $\mathrm{mm}$ diameter) denote regions used to generate the temporal traces of mito-Ca ${ }^{2+}$ signals

1030 in $E^{\prime \prime}$ : adjacent to the presynapse ("1"), and midbody (" 2 " and " 3 ") in the same cell as E. F,

1031 Average evoked mito-Ca ${ }^{2+}$ response before (solid black) and after 30 min incubation with $10 \mu \mathrm{M}$ 
1032 Ru360 (dashed green), $2 \mu \mathrm{M}$ Ru360 (green), or $10 \mu \mathrm{M}$ isradipine (gray) (3-5 dpf, $\mathrm{n} \geq 9$ cells per

1033 treatment). Error bars in D are min and max; in F the shaded area denotes SEM. Scale bar $=500$

$1034 \mathrm{~nm}$ in $\mathrm{C}, 5 \mu \mathrm{m}$ in $\mathrm{B}$ and $2 \mu \mathrm{m}$ in $\mathrm{E}$ and $\mathrm{E}^{\prime}$. 

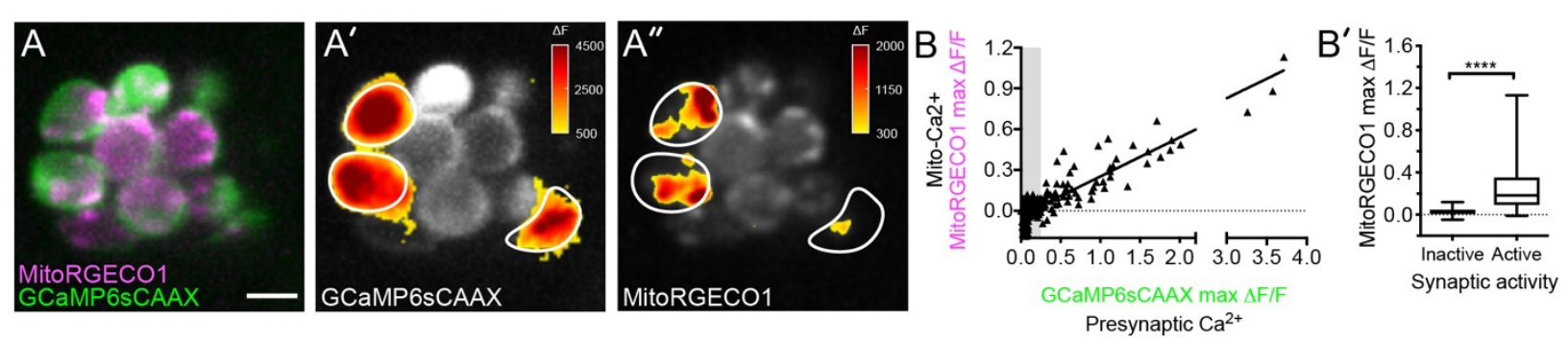

C
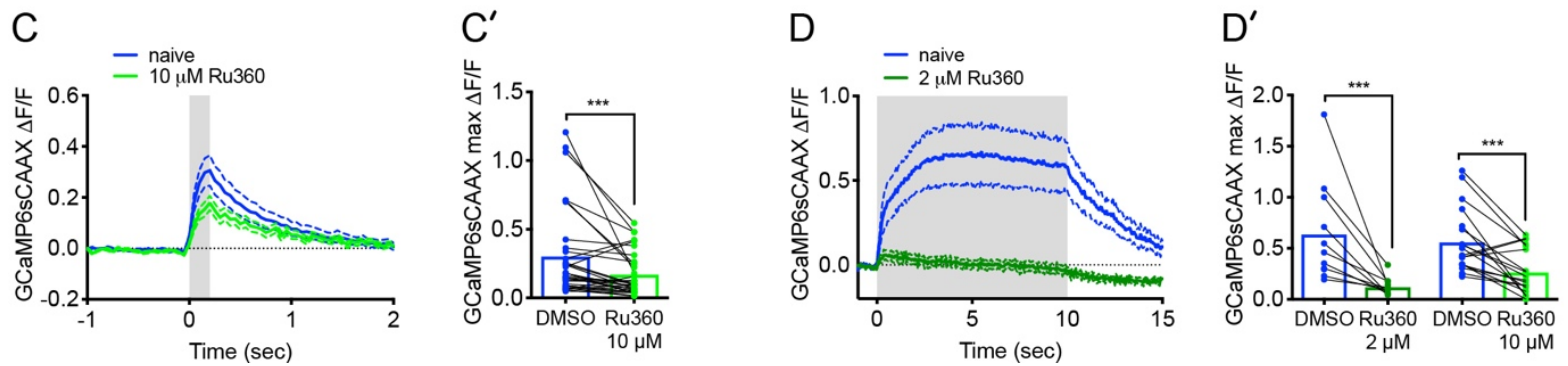

Figure 2. Mito- $\mathrm{Ca}^{2+}$ uptake can impact presynaptic $\mathrm{Ca}^{2+}$ signals. A, A live Image of a neuromast

1037 viewed top-down, expressing the presynaptic-Ca ${ }^{2+}$ sensor GCaMP6sCAAX (green) and mito-Ca ${ }^{2+}$

1038 sensor MitoRGECO1 (magenta) at $3 \mathrm{dpf}$. The GCaMP6sCAAX ( $\left.A^{\prime}\right)$ and MitoRGECO1 ( $\left.A^{\prime \prime}\right)$ signals

1039 during a 2-s stimulation are indicated by the heatmaps and occur in the same cells (white

1040 outline). B, Scatterplot with linear regression of peak presynaptic- and mito-Ca ${ }^{2+}$ response for

1041 individual cells at 3-5 dpf, $n=209$ cells. Gray background in graph denotes presynaptic-Ca ${ }^{2+}$

1042 signals below 0.25 , a threshold used as a cutoff for presynaptic activity (below inactive, above

1043 active). B', Plot of mito-Ca ${ }^{2+}$ responses segregated based on the activity threshold in B. C-D',

1044 Presynaptic-Ca ${ }^{2+}$ response (example in Figure S2) averaged per cell before (blue) and after 30

1045 min of $10 \mu \mathrm{M}$ Ru360 (light green) or $2 \mu \mathrm{M}$ Ru360 (dark green), $\mathrm{n} \geq 10$ cells per treatment. C and

1046 D show averaged traces while $C^{\prime}$ and $D^{\prime}$ show before-and-after dot plots of the peak response

1047 per cell. Whiskers on plots in $\mathrm{B}^{\prime}$ represent min and max; error (dashed lines) in plots C and D

1048 represent SEM. Mann-Whitney $U$ test was used in B'; Wilcoxon matched-pairs signed-rank test

1049 was used in $C^{\prime}$ and $D^{\prime} .{ }^{* * *} p<0.001,{ }^{* * *} p<0.0001$. Scale bar $=5 \mu \mathrm{m}$ in A.

1050

1051 

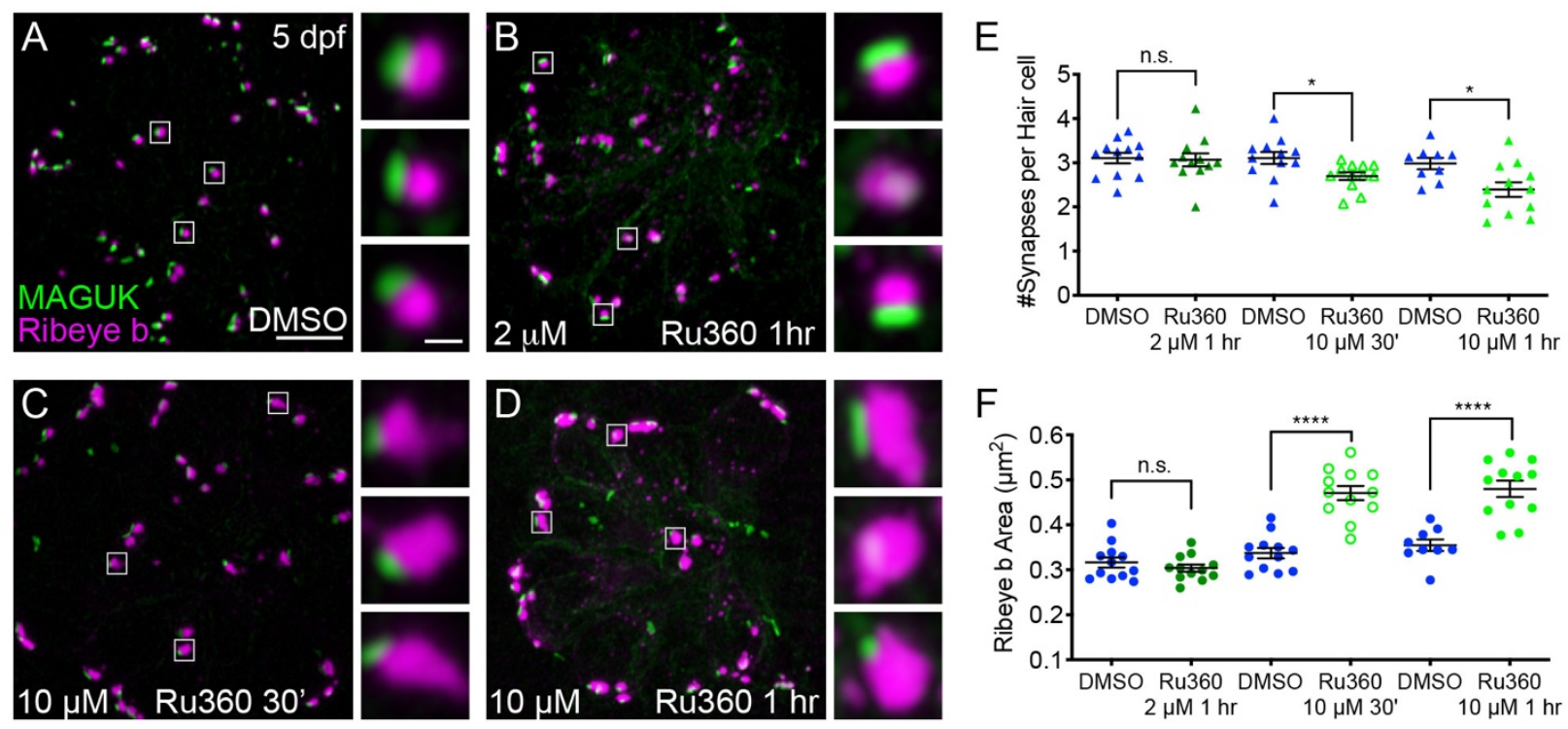

Figure 3. Mito- $\mathrm{Ca}^{2+}$ is important for ribbon size and synapse integrity in mature hair cells. A-D, Representative images of mature neuromasts ( $5 \mathrm{dpf}$ ) immunostained with Ribeye $b$ (magenta, ribbons) and MAGUK (green, postsynapses) after a $1 \mathrm{hr} 0.1 \%$ DMSO (A), a $1 \mathrm{hr} 2 \mu \mathrm{M}$ Ru360 (B), a 30 min $10 \mu \mathrm{M}$ Ru360 (C), or a 1 hr $10 \mu \mathrm{M}$ Ru360 (D) treatment. Insets show 3 example synapses (white squares). E-F, Scatter plots show synapse counts (E), and ribbon area (F) in controls and in treatment groups. $\mathrm{N} \geq 9$ neuromasts per treatment. Error bars in E-F represent SEM. A Welch's unequal variance $t$-test was used in E-F. ${ }^{*} p<0.05, * * * * p<0.0001$. Scale bar $=5$ $\mu \mathrm{m}$ in $A$, and $2 \mu \mathrm{m}$ in inset. 

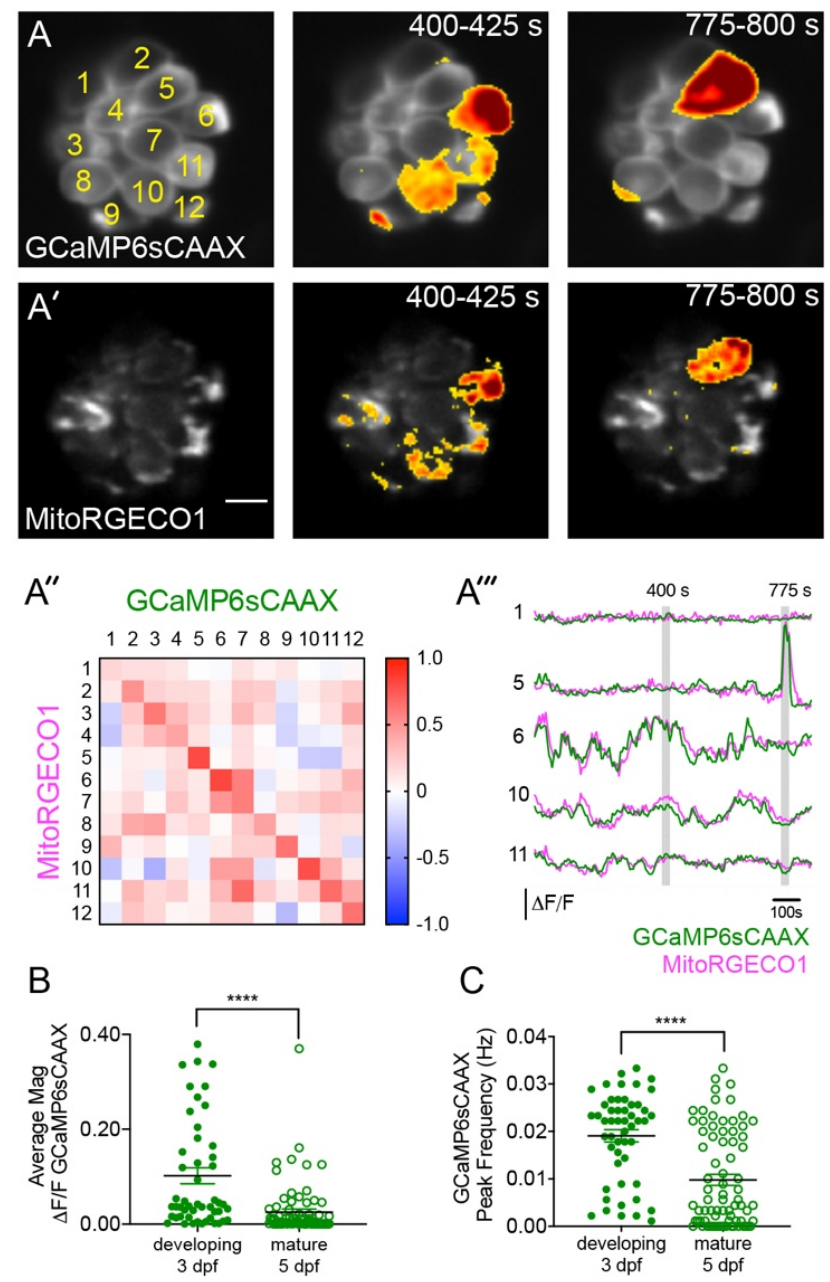

C

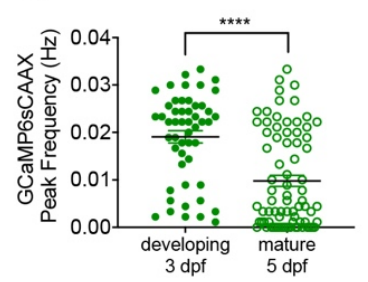

1062 Figure 4. Spontaneous presynaptic- $\mathrm{Ca}^{2+}$ influx and Mito-Ca ${ }^{2+}$ uptake are linked.

1063 A-A', A live Image of a neuromast viewed top-down, expressing the presynaptic-Ca ${ }^{2+}$ sensor

1064 GCaMP6sCAAX (A) and mito-Ca ${ }^{2+}$ sensor MitoRGECO1 $\left(\mathrm{A}^{\prime}\right)$ at $3 \mathrm{dpf}$. Example GCaMP6sCAAX ( $\left.\mathrm{A}^{\prime}\right)$

1065 and MitoRGECO1 ( $\mathrm{A}^{\prime}$ ) signals during two 25-s windows within a 900-s acquisition are indicated

1066 by the $\Delta \mathrm{F}$ heatmaps and occur in the same cells. $\mathrm{A}^{\prime \prime}, \mathrm{A}$ heatmap of Pearson correlation

1067 coefficients comparing GCaMP6sCAAX and MitoRGECO1 signals from the cells in A-A'. $A^{\prime \prime \prime}$,

1068 Example GCaMP6sCAAX (green) MitoRGECO1 (magenta) traces during the 900-s acquisition

1069 from the 5 cells numbered in A, also see Movie S2. B, Scatterplot showing the average

1070 magnitude of GCaMP6sCAAX signals in developing and mature hair cells, $\mathrm{n}=6$ neuromasts per

1071 age. C, Scatterplot showing frequency of GCaMP6sCAAX events in developing and mature hair

1072 cells, $n=6$ neuromasts. Error bars in B-C represent SEM. A Mann-Whitney $U$ test was used in B

1073 and $C .{ }^{* * *} p<0.0001$. Scale bar $=5 \mu \mathrm{m}$ in A. 

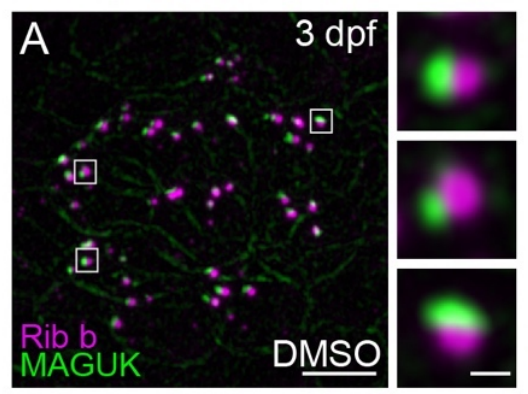

$\mathrm{D}$

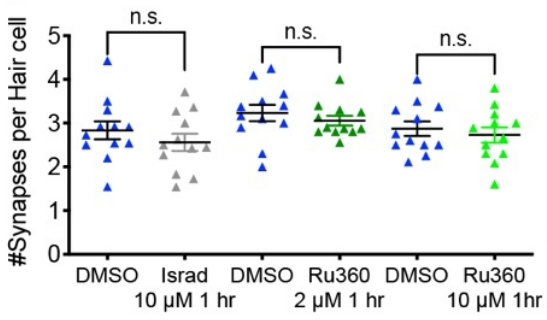

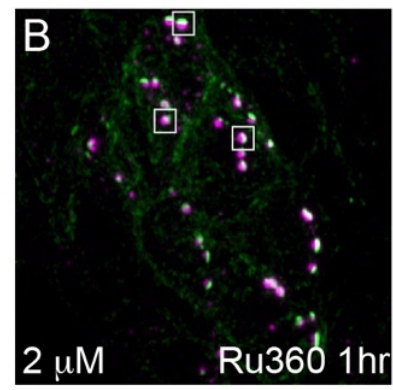

$\mathrm{E}$

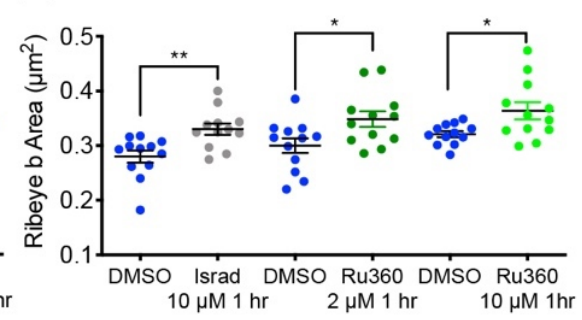

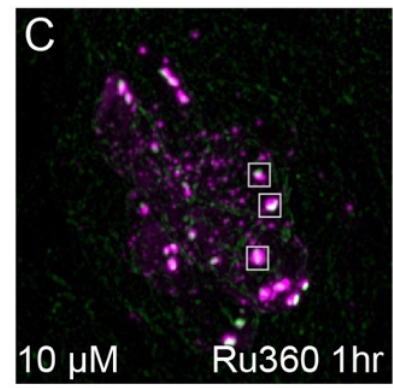
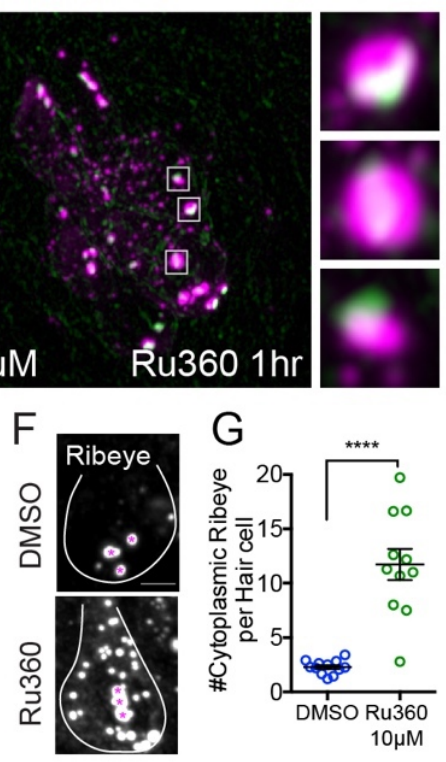

Figure 5. Mito- $\mathrm{Ca}^{2+}$ regulates ribbon formation. A-C, Representative images of immature

1076 neuromasts (3 dpf) immunostained with Ribeye b (magenta, ribbons) and MAGUK (green,

1077 postsynapses) after a 1 hr 0.1\% DMSO (A), $2 \mu \mathrm{M}$ Ru360 (B) or $10 \mu \mathrm{M}$ Ru360 (C) treatment.

1078 Insets show 3 representative synapses (white squares) for each treatment. (D-E) Scatterplot

1079 show quantification of synapse number (D), and ribbon area (E) in controls and in treatment

1080 groups. F, Side-view of hair cell (white outline) shows synaptic ribbon (magenta asterisks) and

1081 extrasynaptic Ribeye b aggregates after a $1 \mathrm{hr} 0.1 \%$ DMSO or $10 \mu \mathrm{M}$ Ru360 treatment.

1082 Quantification of extrasynaptic Ribeye puncta (G). N $\geq 12$ neuromasts per treatment. Error bars

1083 in B-C represent SEM. Welch's unequal variance $t$-test was used in D-E and G, ${ }^{*} p<0.05,{ }^{* *} p<$

$10840.01,{ }^{* * * *} p<0.0001$. Scale bar $=5 \mu \mathrm{m}$ in $\mathrm{A}, 2 \mu \mathrm{m}$ in insets and $\mathrm{F}$. 

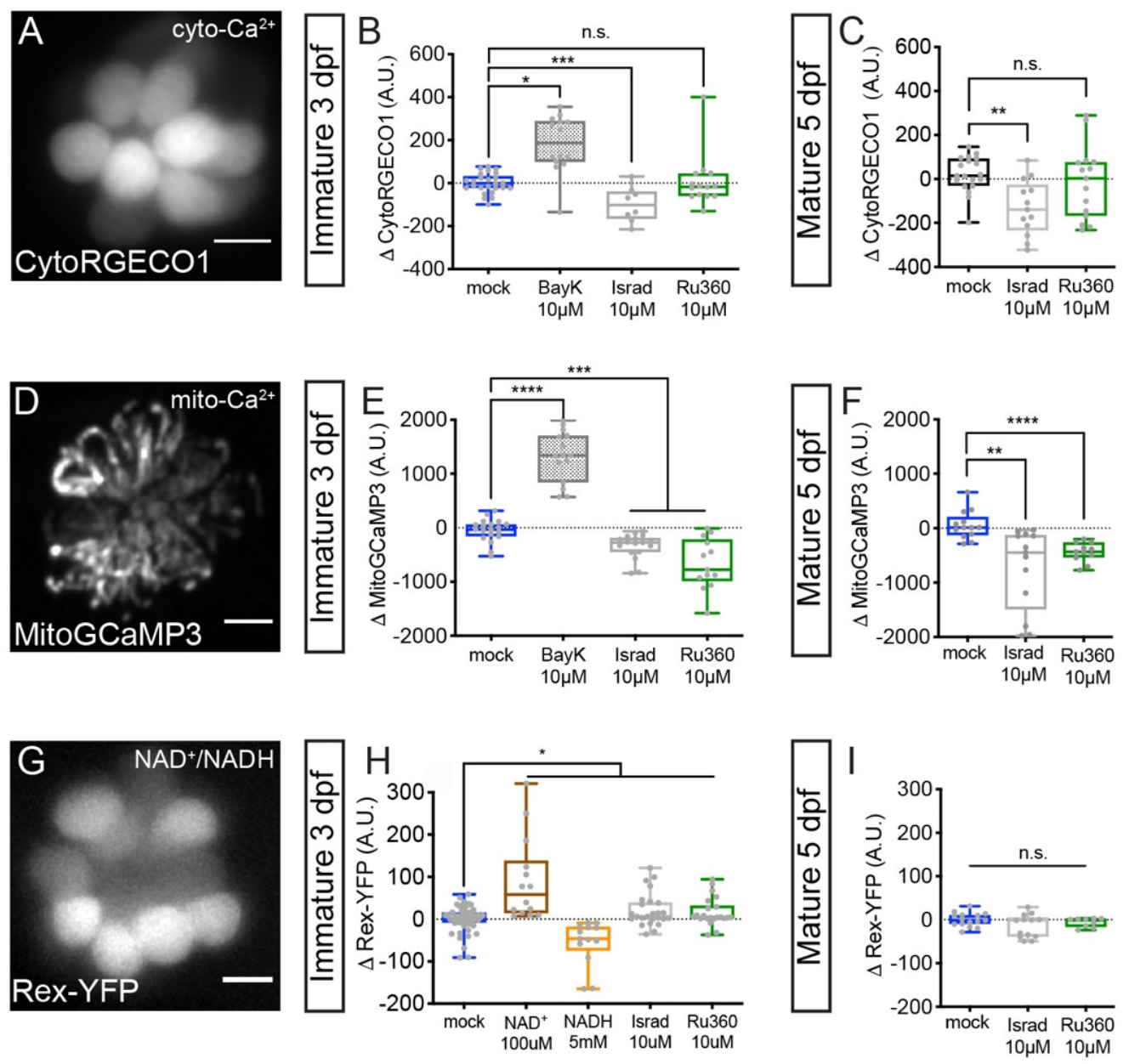

Figure 6. Cyto- $\mathrm{Ca}^{2+}$, mito- $\mathrm{Ca}^{2+}$ and $\mathrm{NAD}^{+} / \mathrm{NADH}$ redox baseline measurements. Live hair cells expressing RGECO1 (A), MitoGCaMP3 (D), or Rex-YFP (G) show resting cyto-Ca ${ }^{2+}$, mito-Ca ${ }^{2+}$ or $\mathrm{NAD}^{+} / \mathrm{NADH}$ levels respectively. B-C, RGECO1 baseline measurements before and after a $30 \mathrm{~min}$

1090 mock treatment (0.1\% DMSO), or after a 30 min $10 \mu \mathrm{M}$ Bay K8644 (BayK), $10 \mu \mathrm{M}$ isradipine, or

$109110 \mu \mathrm{M}$ Ru360 treatment. E-F, MitoGCaMP3 baseline measurements before and after a $30 \mathrm{~min}$

1092 mock treatment (0.1\% DMSO), or after a $10 \mu \mathrm{M}$ BayK, $10 \mu \mathrm{M}$ isradipine, or $10 \mu \mathrm{M}$ Ru360

1093 treatment. H-I, Rex-YFP baseline measurements before and after 30 min mock treatment $(0.1 \%$

1094 DMSO), or after a 30 min $100 \mu \mathrm{M} \mathrm{NAD}^{+}, 5$ mM NADH, $10 \mu \mathrm{M}$ isradipine, or $10 \mu \mathrm{M}$ Ru360

1095 treatment. All plots are box-and-whiskers plot that show median, min and max. $\mathrm{N} \geq 9$

1096 neuromasts per treatment. One-way Brown-Forsythe and Welch ANOVA with Dunnett's T3 post

1097 hoc was used to calculate the difference in $\mathrm{B}-\mathrm{C}, \mathrm{E}-\mathrm{F}$, and $\mathrm{H}-\mathrm{I},{ }^{*} p<0.05,{ }^{* *} p<0.01,{ }^{* * *} p<0.001$, $1098{ }^{* * * *} p<0.0001$. Scale bar $=5 \mu \mathrm{m}$ in A, D and G. 

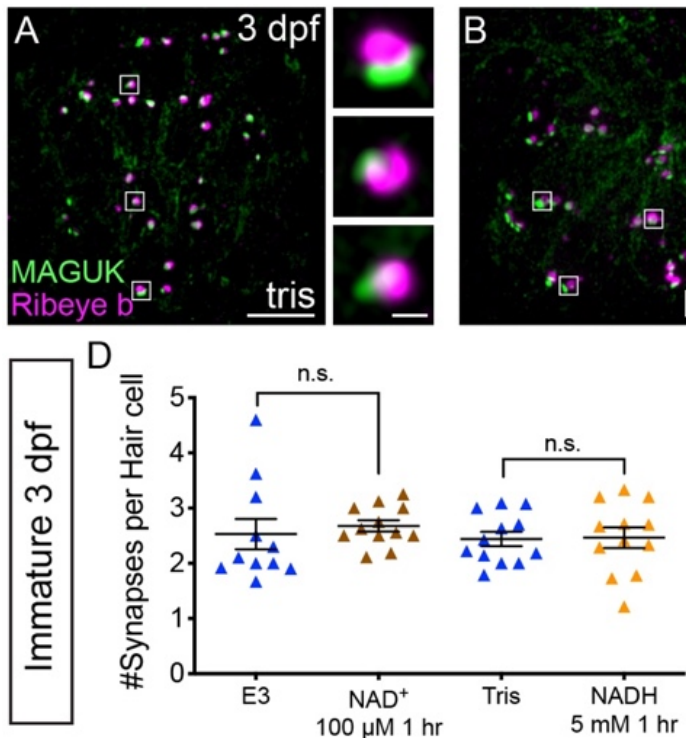
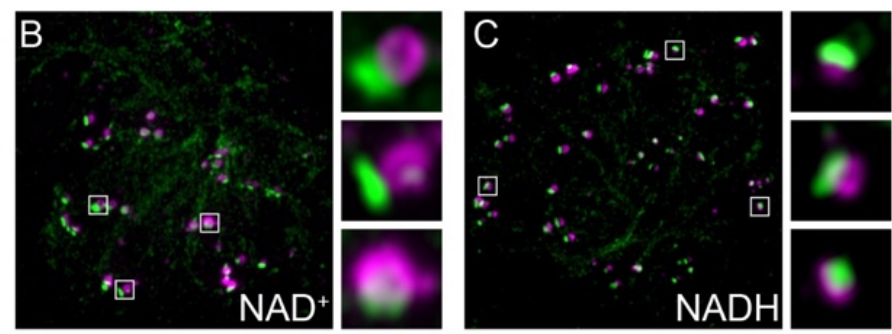

$\mathrm{E}$

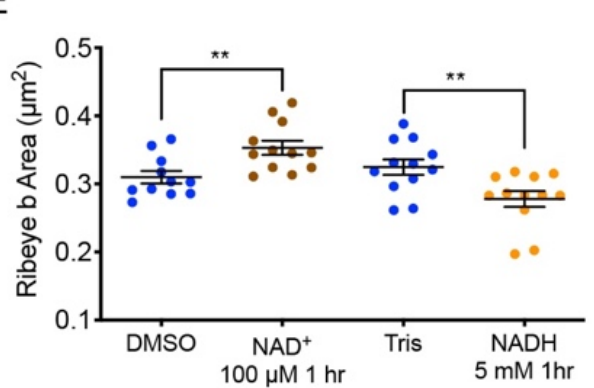

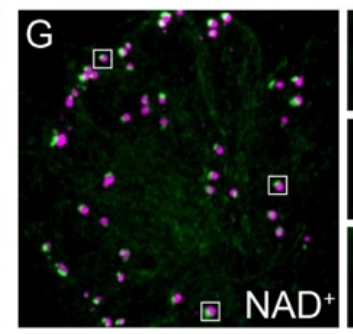
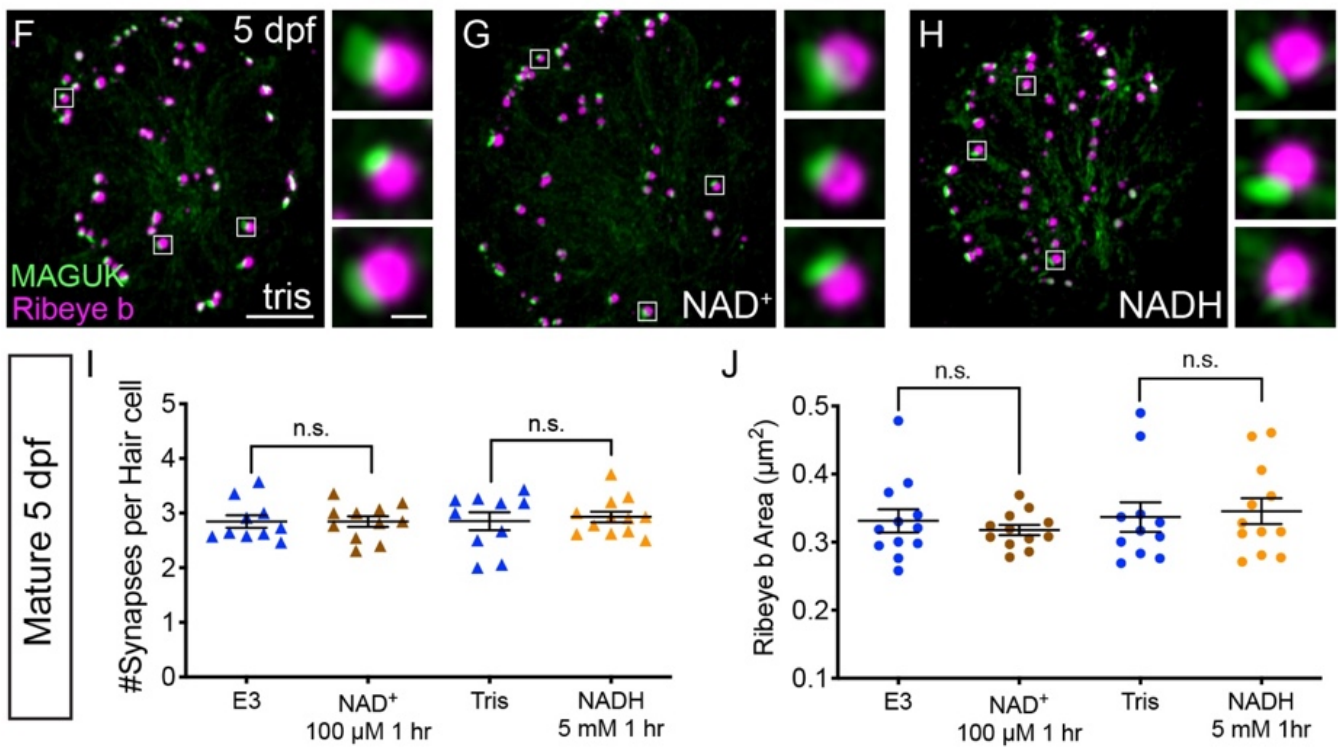

Figure 7. $\mathrm{NAD}^{+}$and $\mathrm{NADH}$ directly influence ribbon formation. Representative images of

1102 immature (A-C, $3 \mathrm{dpf})$ and mature (G-H, $5 \mathrm{dpf}$ ) neuromasts immunostained with Ribeye $b$

1103 (magenta, ribbons) and MAGUK (green, postsynapses) after a 0.1\% Tris- $\mathrm{HCl}(\mathrm{A}, \mathrm{F}), 100 \mu \mathrm{M}$ NAD ${ }^{+}$

$1104(B, G)$ or 5 mM NADH treatment $(C, H)$. Insets show 3 example synapses (white squares). D-E

1105 and I-J, Scatterplots show synapse count $(D, I)$ and ribbon area $(E, J)$ in controls and treatments

1106 groups. $\mathrm{N} \geq 10$ neuromasts per treatment. Error bars in B-C represent SEM. A Welch's unequal

1107 variance $t$-test was used for comparisons, ${ }^{* *} p<0.01$. Scale bar $=5 \mu \mathrm{m}$ in $\mathrm{A}$ and $\mathrm{F}, 2 \mu \mathrm{m}$ in insets. 
A Developing hair cell

Spontaneous

presynaptic influx

\& Mito-Ca ${ }^{2+}$ uptake

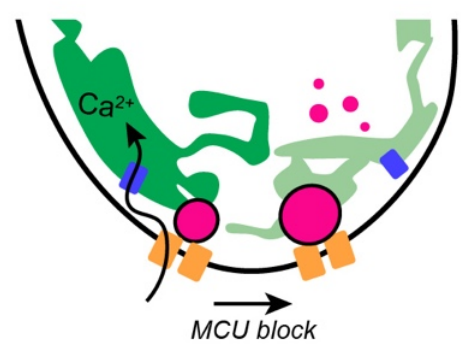

Partial or complete MCU block ribbon enlargement via $\operatorname{NAD}(\mathrm{H})$ redox
B Mature hair cell

Evoked

presynaptic influx

\& Mito-Ca ${ }^{2+}$ uptake

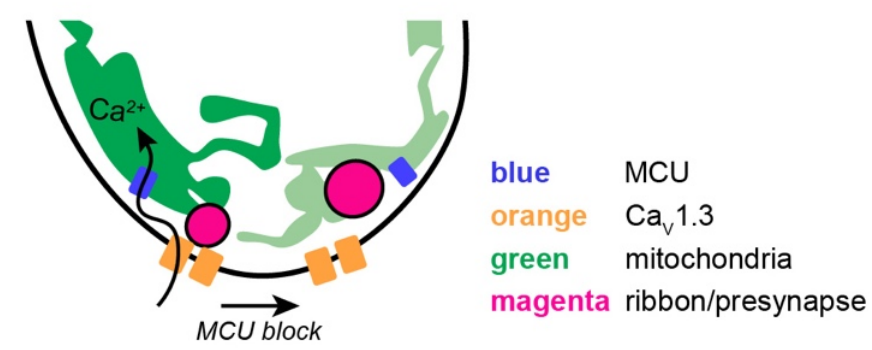

Partial MCU block presynaspe function loss
Complete MCU block ribbon enlargement synapse loss

1110 Figure 8. Schematic model of mito- $\mathrm{Ca}^{2+}$ in developing and mature hair cells. A, In developing 1111 hair cells, spontaneous presynaptic- $\mathrm{Ca}^{2+}$ influx is linked to mito- $\mathrm{Ca}^{2+}$ uptake. Together these $1112 \mathrm{Ca}^{2+}$ signals function to regulate ribbon formation. When the Cav1.3 or MCU channels are 1113 blocked, ribbon formation is increased leading to larger ribbons. These $\mathrm{Ca}^{2+}$ signals regulate 1114 ribbon formation via $\mathrm{NAD}(\mathrm{H})$ redox. $\mathrm{B}$, In mature hair cells, evoked presynaptic- $\mathrm{Ca}^{2+}$ influx is 1115 linked to mito- $\mathrm{Ca}^{2+}$ uptake. When the $\mathrm{MCU}$ is blocked in mature hair cells there are 1116 synaptopathic consequences. Ribbons are enlarged and synapses are lost. 
1118 Movie S1. Airyscan image of MitoGCaMP3 and Rib a-tagRFP at the base of a single live hair cell.

1119 Movie S2. Spontaneous $\triangle F$ GCaMP6sCAAX (left) and $\triangle F$ MitoRGECO1 (right) signals acquired at

11203 dpf, 25-s per frame.

1121

1122

1123

1124

1125

1126

1127

1128

1129

1130

1131

1132

1133

1134

1135 

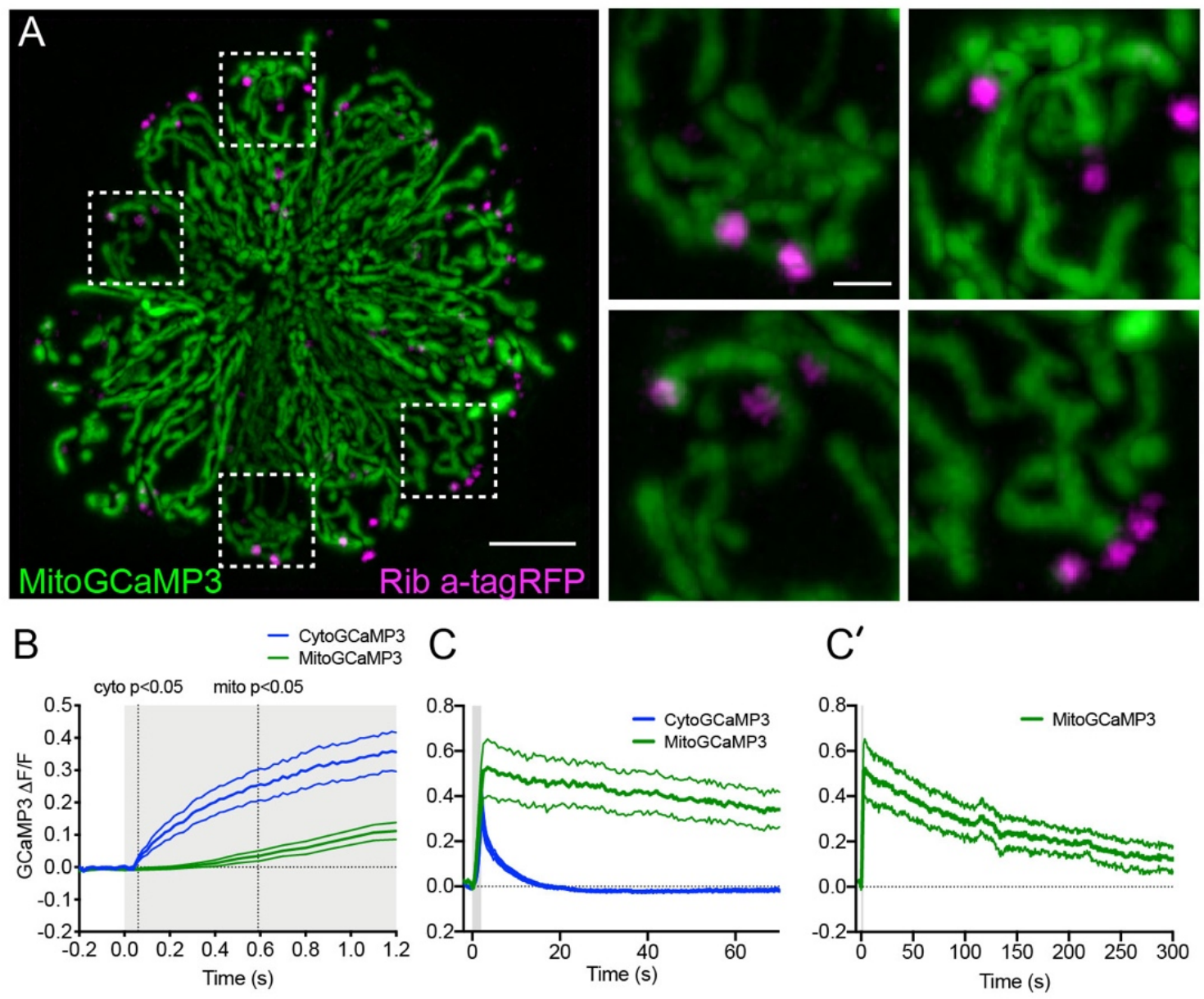

1137 Figure S1. The time course of mechanically-evoked mito-Ca ${ }^{2+}$ and cyto-Ca ${ }^{2+}$ signals are distinct.

1138 A, Airyscan confocal image of a live, neuromast expressing MitoGCaMP3 (mitochondria) and

1139 Ribeye a-tagRFP (ribbons) at $6 \mathrm{dpf}$. Insets show the base of 4 individual hair cells from the

1140 neuromast in $A$ (dashed white boxes). B, Average cyto- (blue) and mito-Ca ${ }^{2+}$ (green) signals

1141 during the onset of a 2-s stimulus. Mito- $\mathrm{Ca}^{2+}$ signals rise with a delay compared to cyto-Ca ${ }^{2+}$

1142 signals (3-6 dpf, $n \geq 18$ cells). C-C', Average cyto- and mito-Ca ${ }^{2+}$ signals during and after a 2-s

1143 stimulation shows that cyto- $\mathrm{Ca}^{2+}$ signals return to baseline shortly after stimulation (C), while

1144 mito- $\mathrm{Ca}^{2+}$ remains elevated up to $5 \mathrm{~min}$ after stimulation ( $\mathrm{C}-\mathrm{C}^{\prime}$ ) ( $3 \mathrm{dpf}, \mathrm{n} \geq 7$ cells). Error in panel

1145 B-C' represent SEM. Scale bar $=5 \mu \mathrm{m}$ in A and $2 \mu \mathrm{m}$ in inset. 

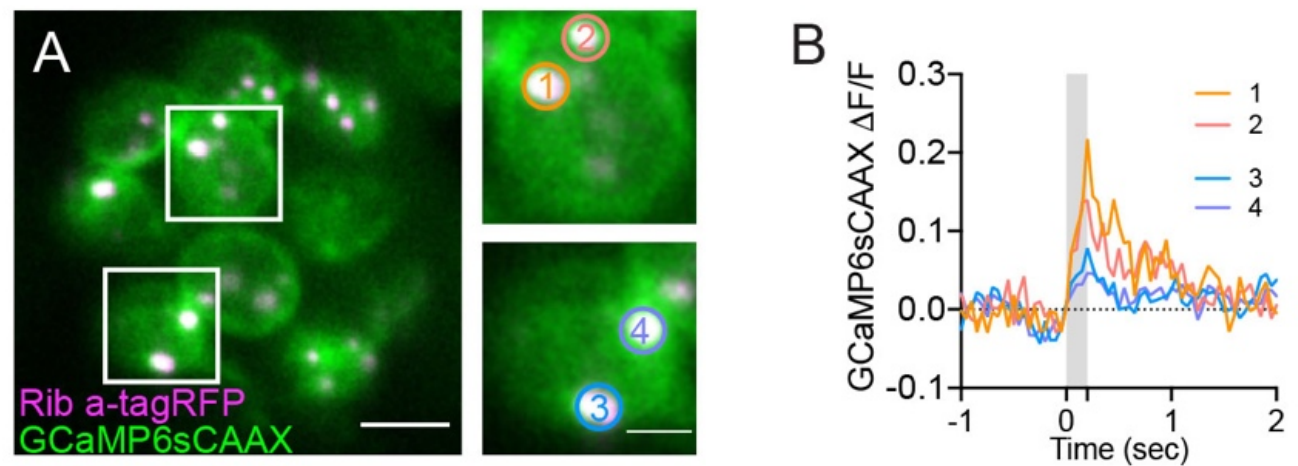

1148 Figure S2. Presynaptic $\mathrm{Ca}^{2+}$ signals at the ribbon synapse. A, Live image of a neuromast viewed

1149 top-down, expressing the presynaptic-Ca ${ }^{2+}$ sensor GCaMP6sCAAX (green) and ribbon label

1150 Ribeye a-tagRFP (magenta) at $3 \mathrm{dpf}$. Example cells show evoked synaptic-Ca ${ }^{2+}$ signals during a

1151 0.2-s stimulation (white boxes, duplicated on right). Circles 1-4 (1.3 $\mu \mathrm{m}$ diameter) denote

1152 regions used to generate the temporal traces of presynaptic-Ca ${ }^{2+}$ signals in $B$. Scale bar $=5 \mu \mathrm{m}$

1153 in $\mathrm{A}$ and $2 \mu \mathrm{m}$ in insets.

1154

1155

1156

1157

1158

1159 
A
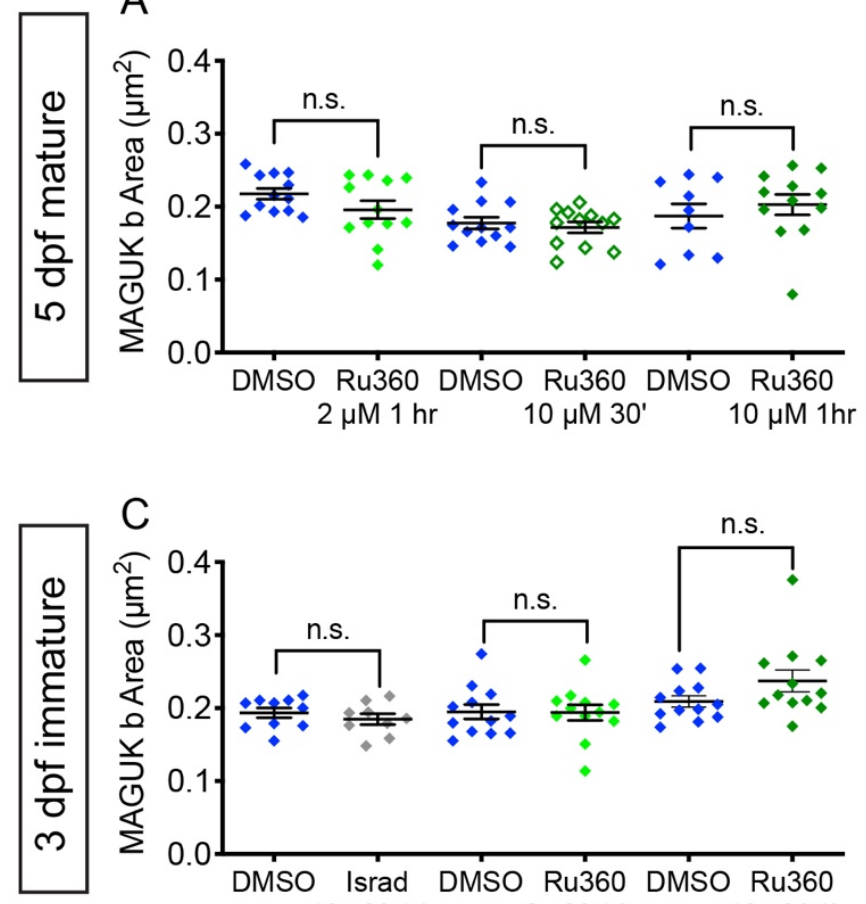

(

C

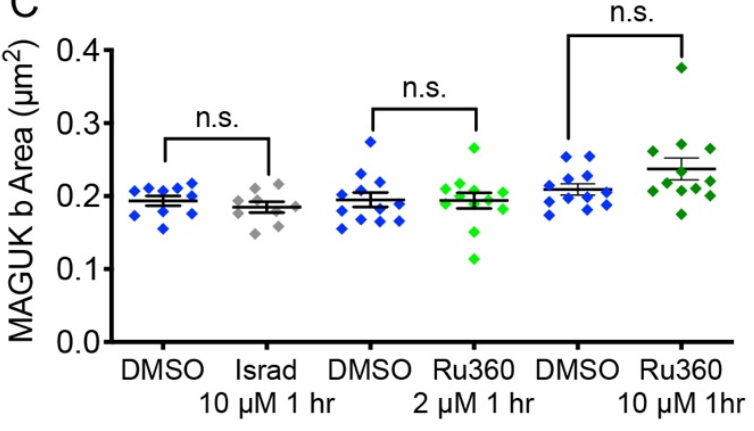

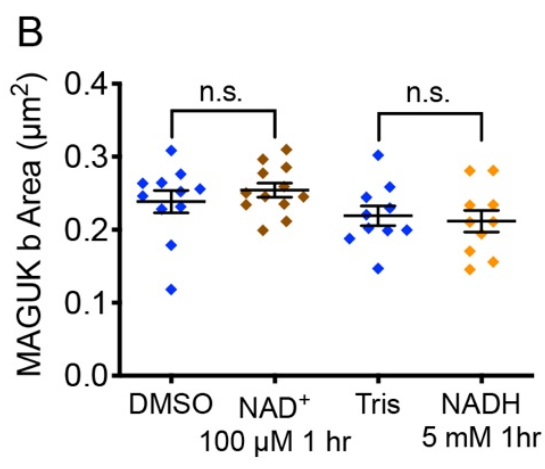

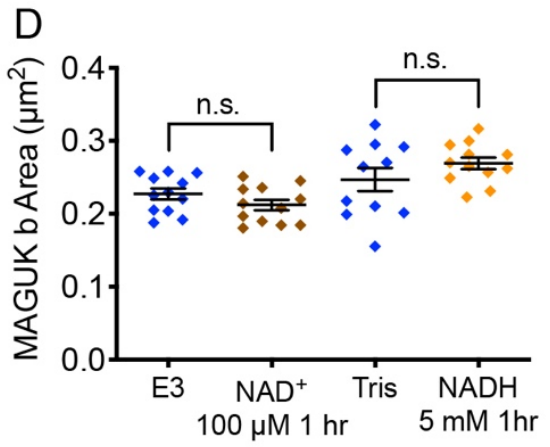

1162 Figure S3. NAD ${ }^{+}$, NADH and Ru360 treatments do not impact postsynapse size. (A-D).

1163 Quantification of postsynapse size assayed by MAGUK immunolabel in mature (A-B) and

1164 developing neuromasts (C-D). Treatments with E3, 0.1\% DMSO, 0.1\% Tris- $\mathrm{HCl}, 100 \mu \mathrm{MAD}^{+}, 5$

1165 mM NADH treatment, $2 \mu \mathrm{M}$ Ru360, $10 \mu \mathrm{M}$ Ru360 do not significantly alter postsynapse size

1166 compared to controls. (C, H). N $\geq 9$ neuromasts per treatment. Error bars in B-C represent SEM.

1167 A Welch's unequal variance $t$-test was used for comparisons. 

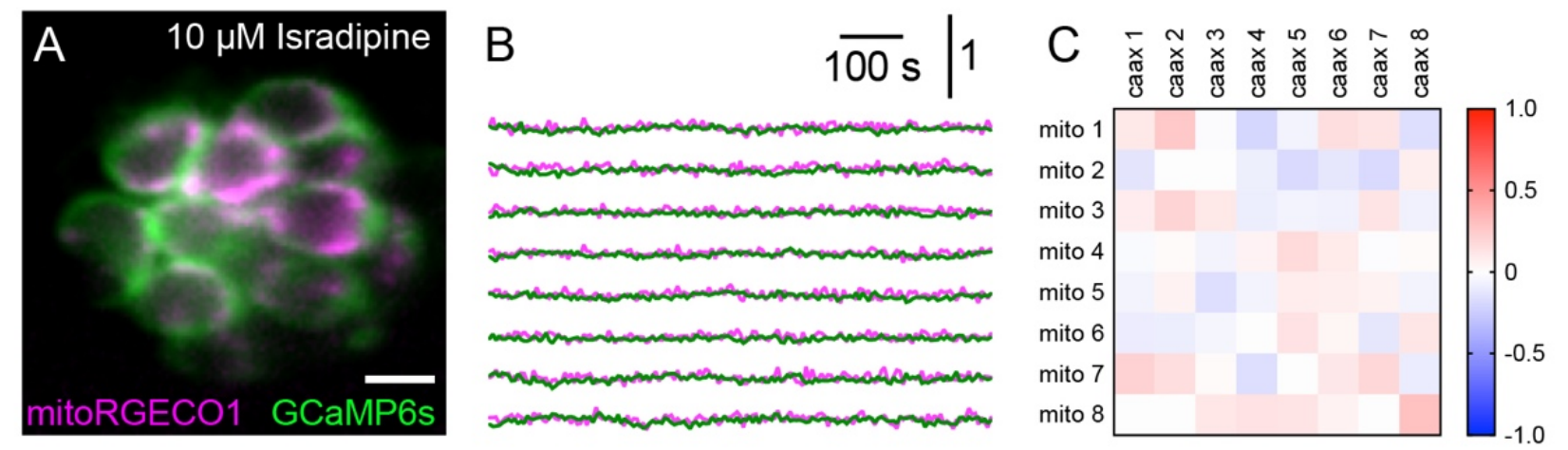

1176 Figure S4. Spontaneous presynaptic and mito-Ca ${ }^{2+}$ signals are abolished by Cav1.3 channel

1177 antagonist isradipine. A, A live Image of a neuromast viewed top-down, expressing the

1178 presynaptic-Ca ${ }^{2+}$ sensor GCaMP6sCAAX (green) and mito-Ca ${ }^{2+}$ sensor MitoRGECO1 (magenta) at

11796 dpf. B, Representative GCaMP6sCAAX (green) and MitoRGECO1 (magenta) traces during a

$1180900-s$ continuous image acquisition in the absence of stimuli and $10 \mu \mathrm{M}$ isradipine. $\mathrm{C}$, There is

1181 no correlation between GCaMP6sCAAX and MitoRGECO1 signals within each cell in the

1182 presence of isradipine. 


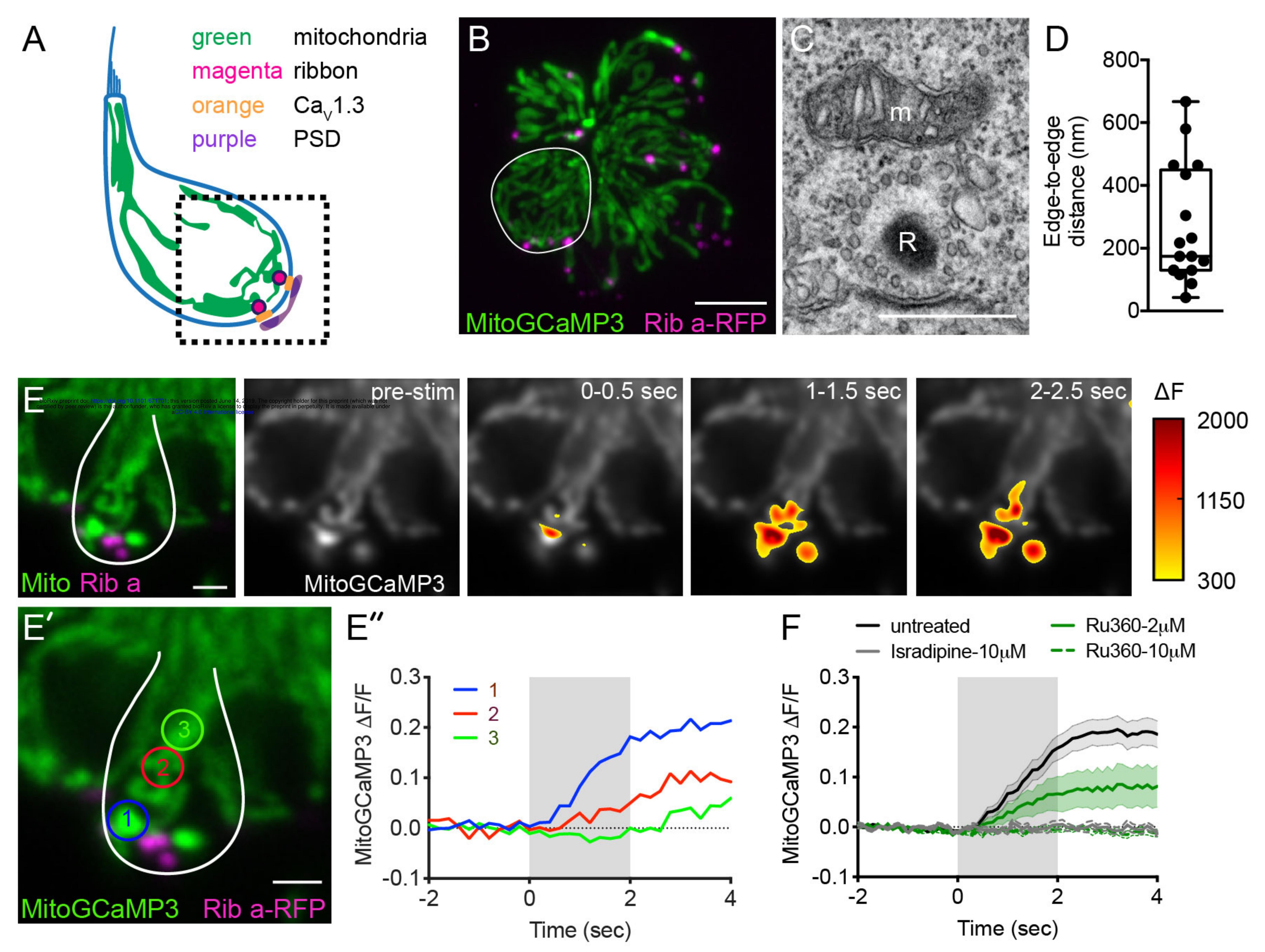



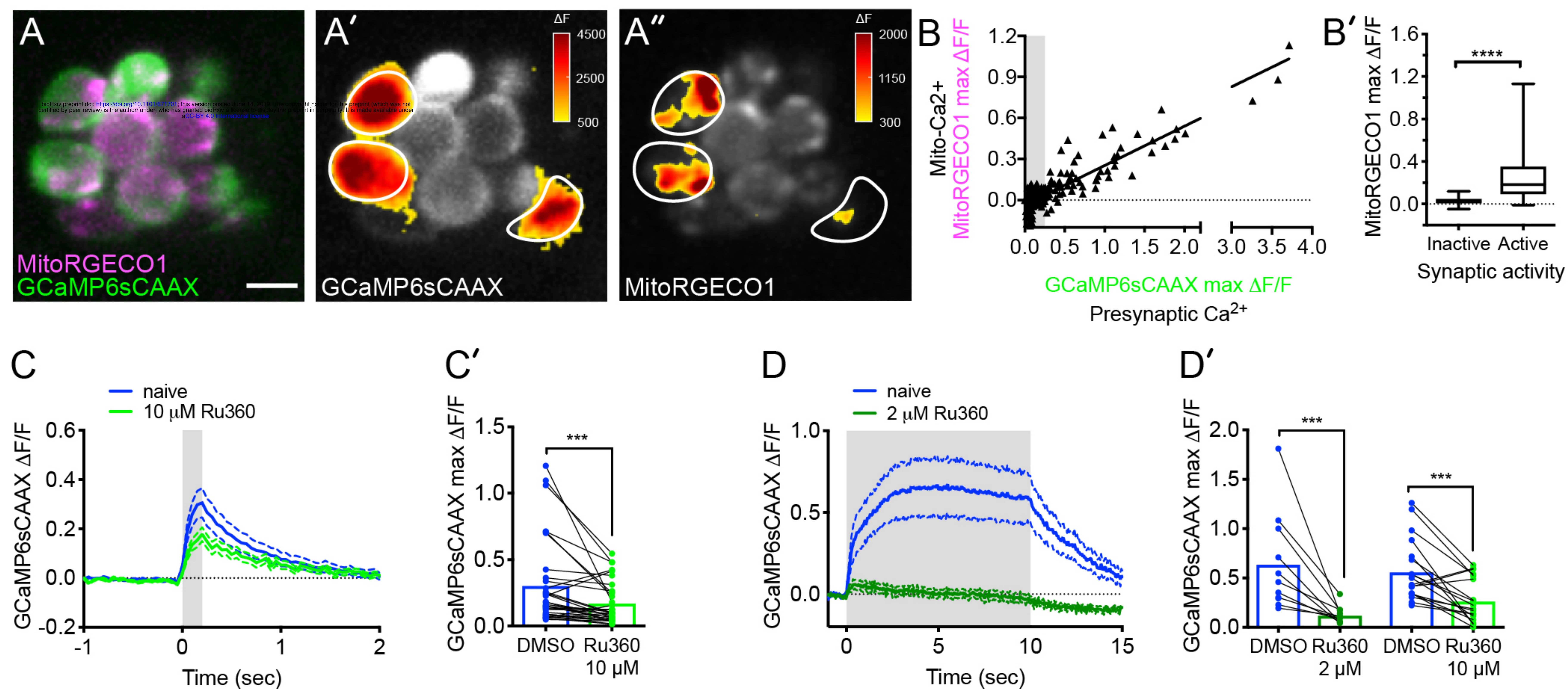


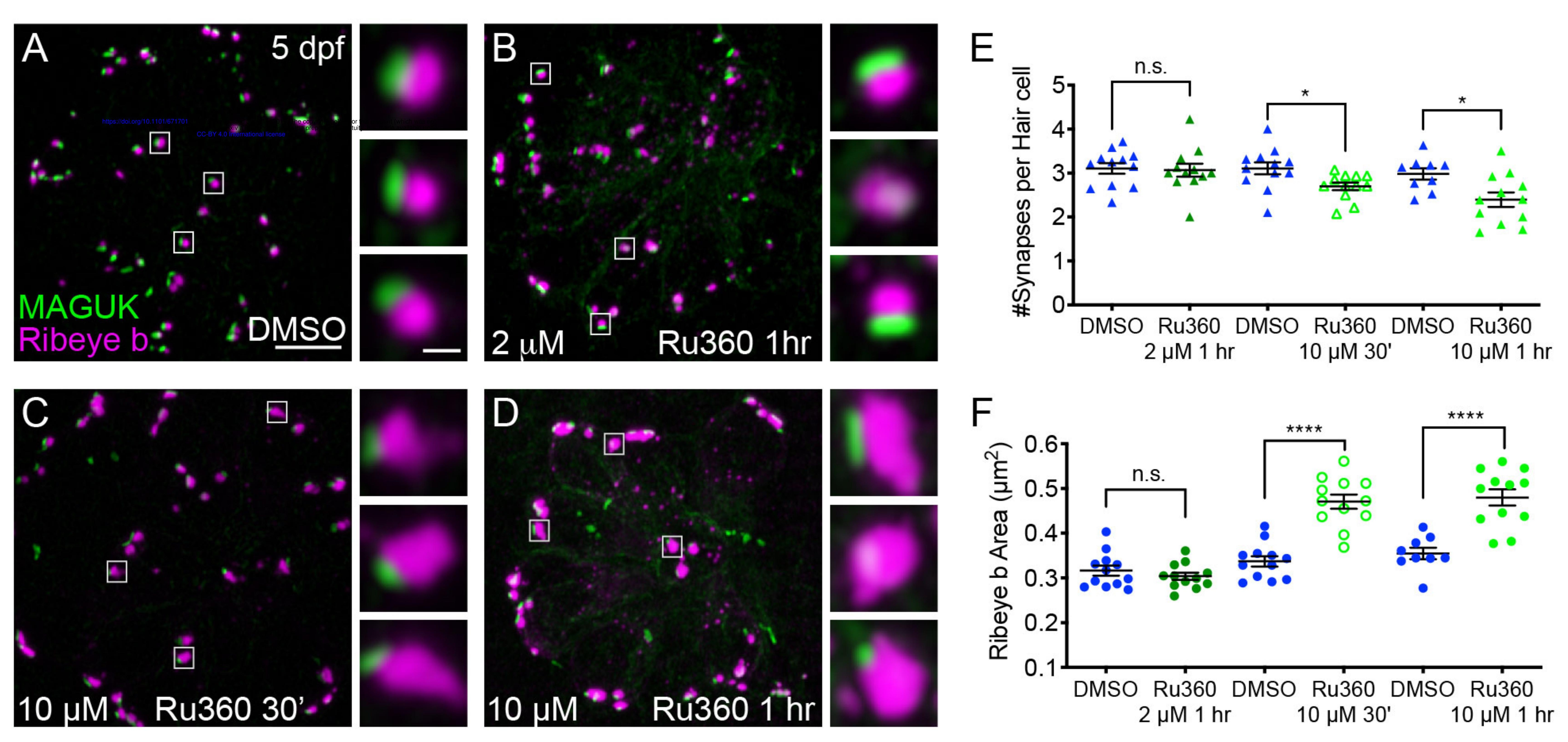



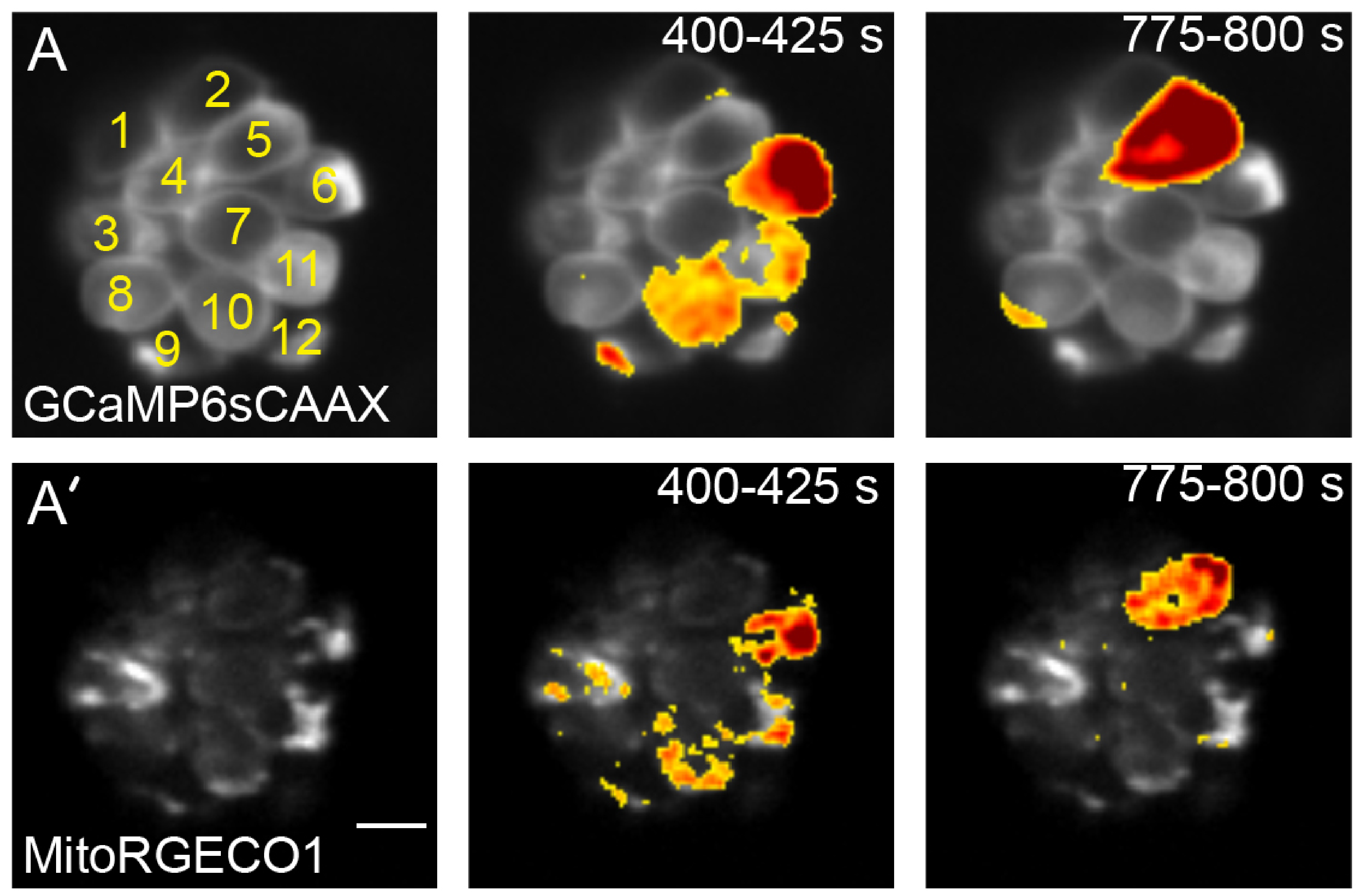

$\mathrm{A}^{\prime \prime}$

GCaMP6sCAAX

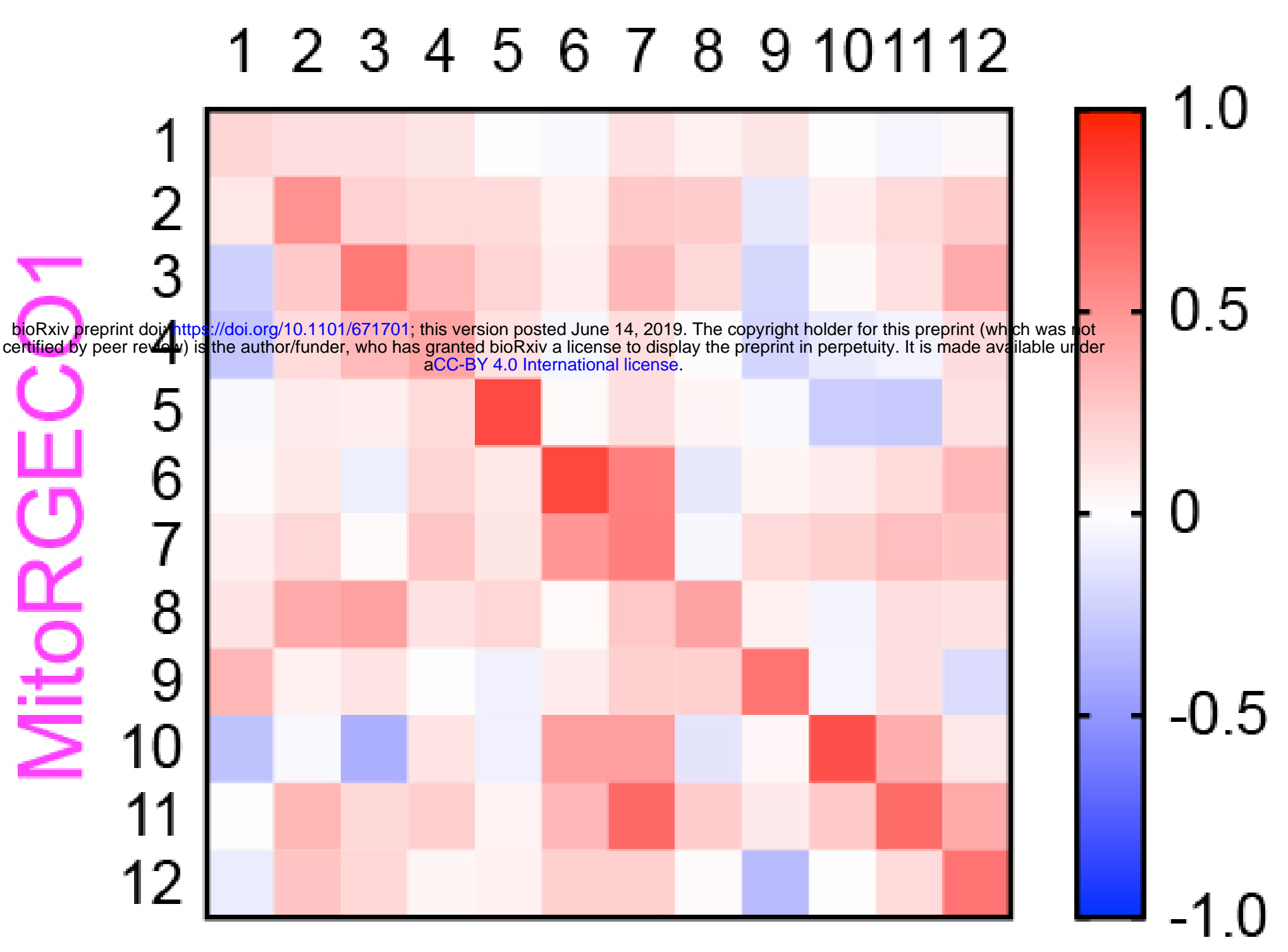

$A^{\prime \prime \prime}$

$400 \mathrm{~s}$

775 s

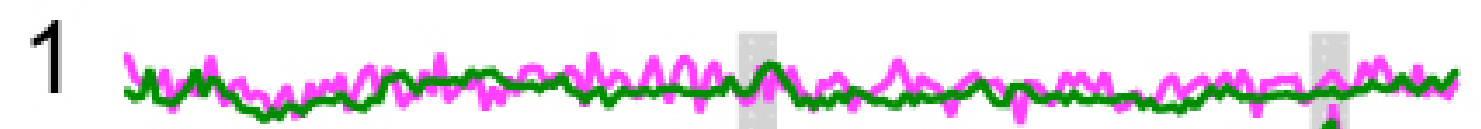

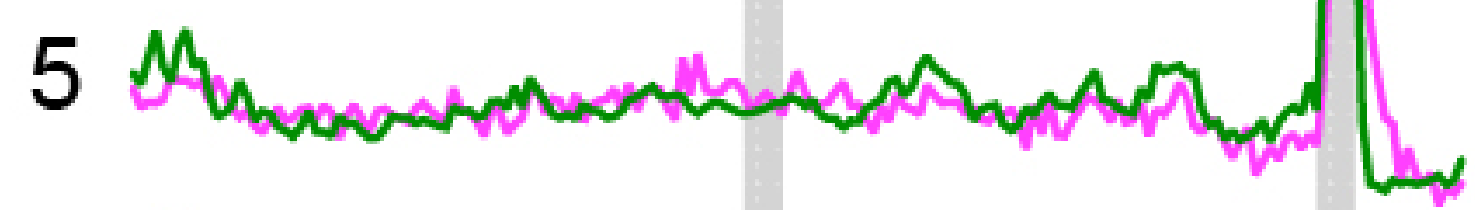

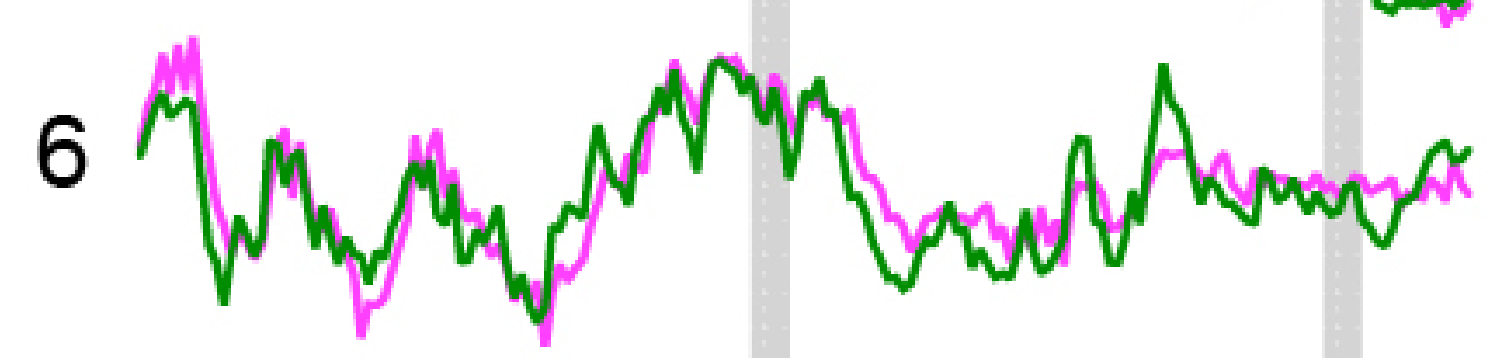

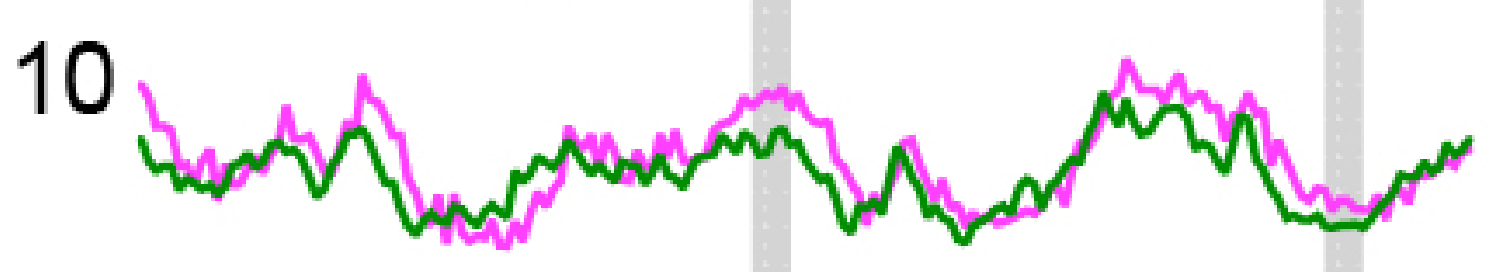

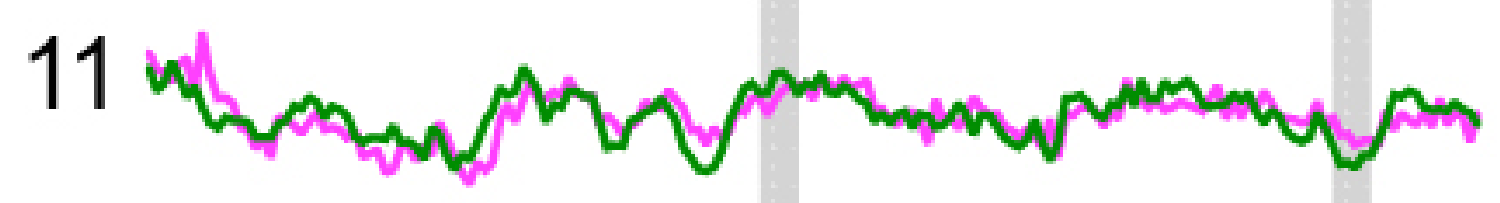
$\Delta \mathrm{F} / \mathrm{F}$ $\overline{100 s}$ GCaMP6sCAAX

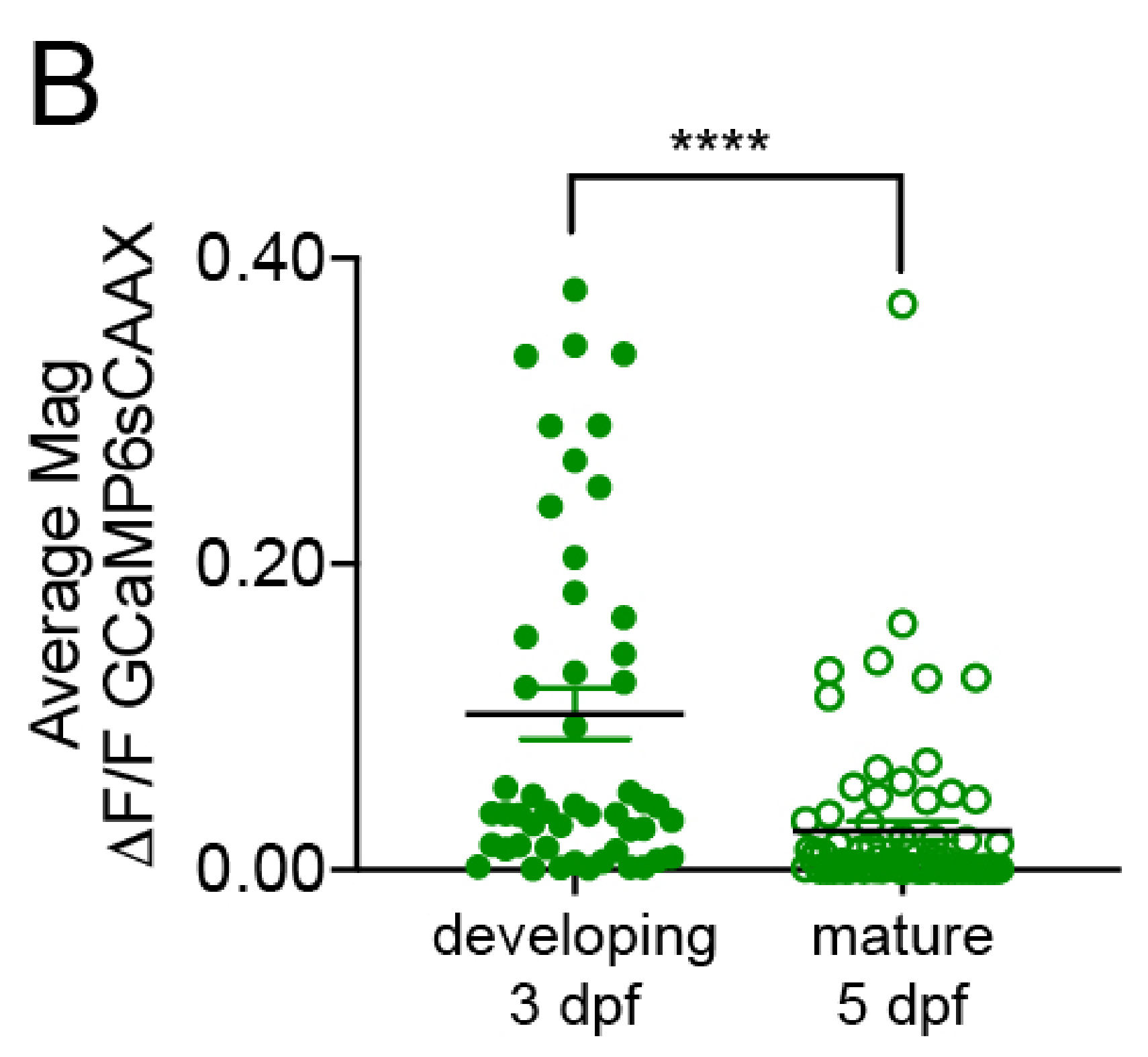

C MitoRGECO1

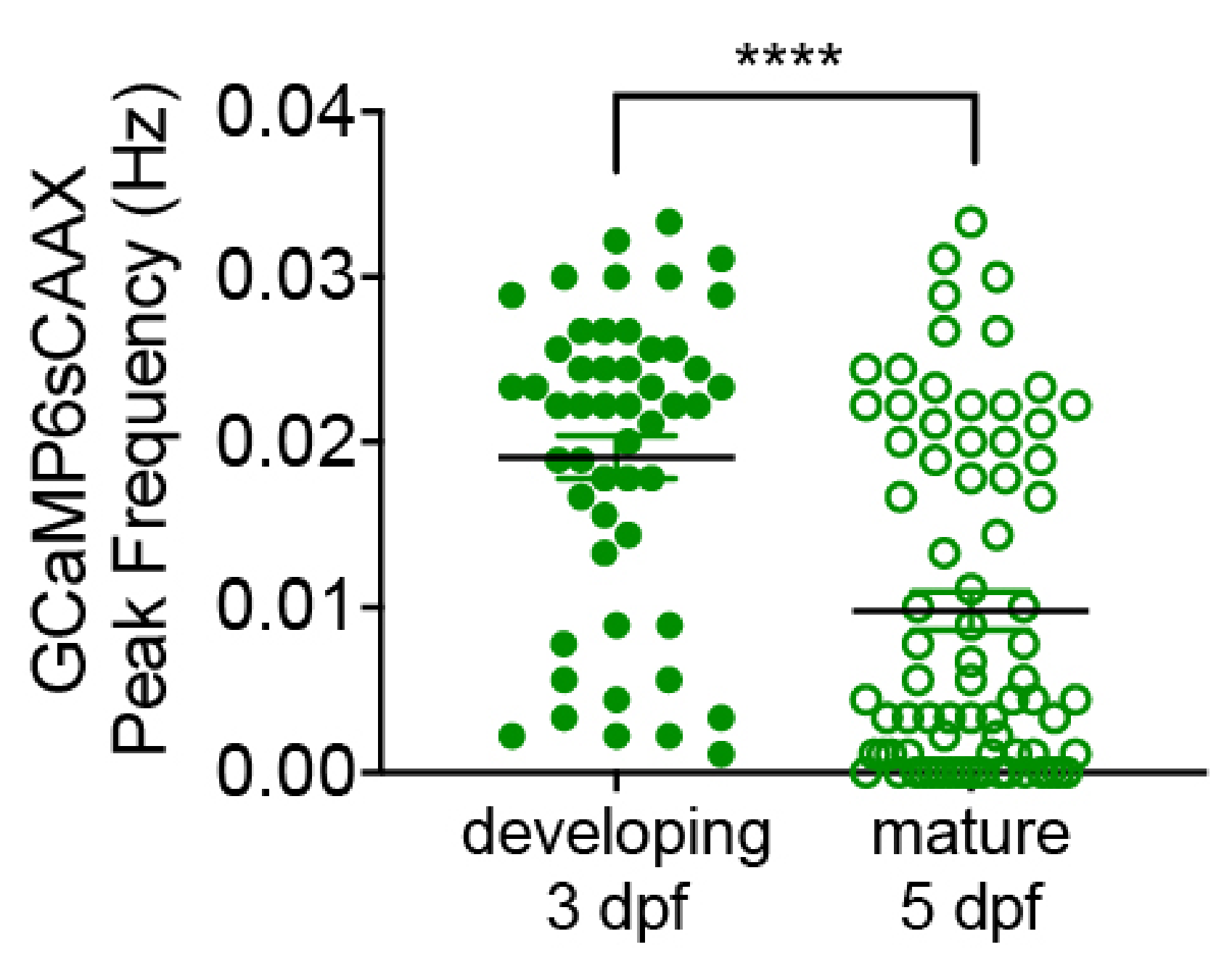




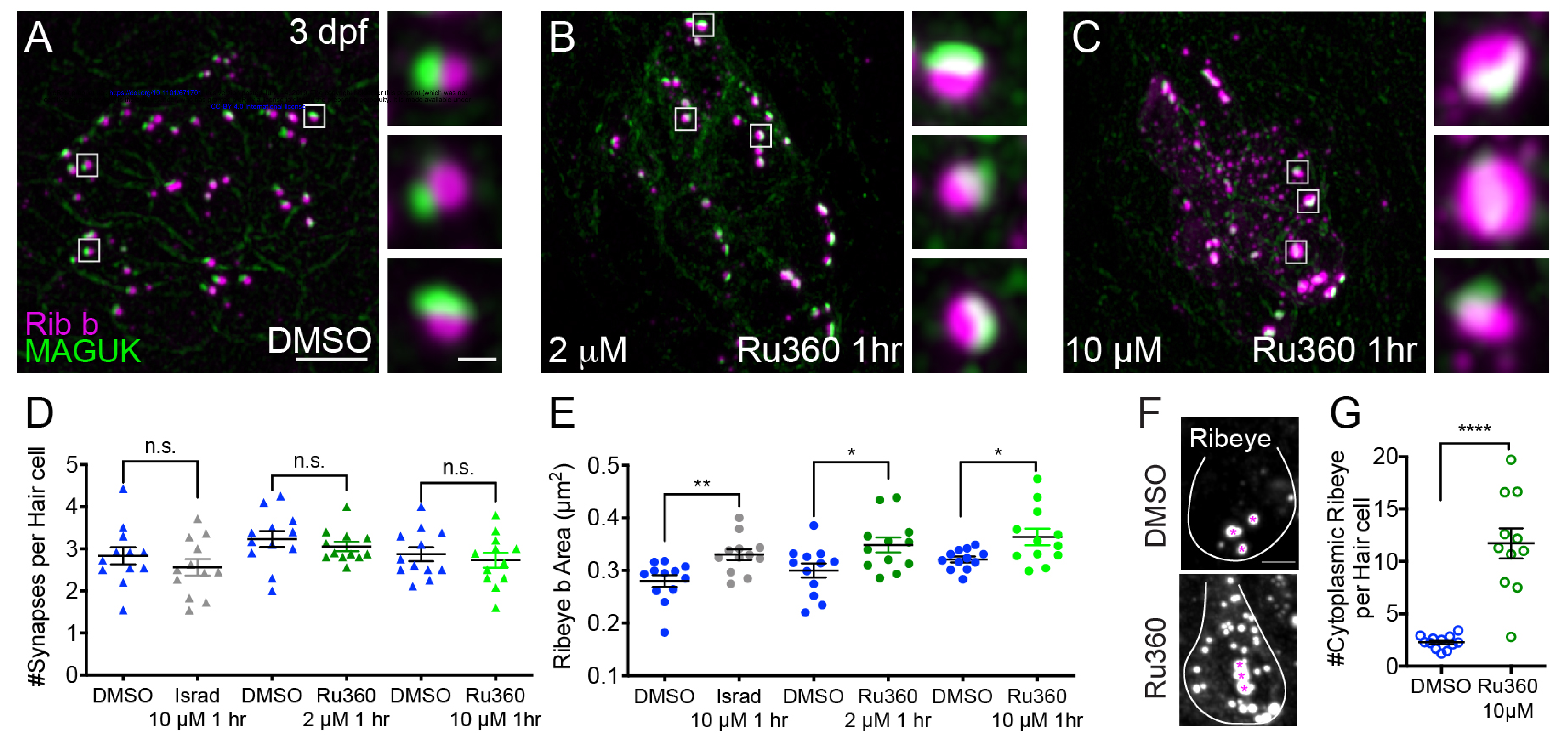



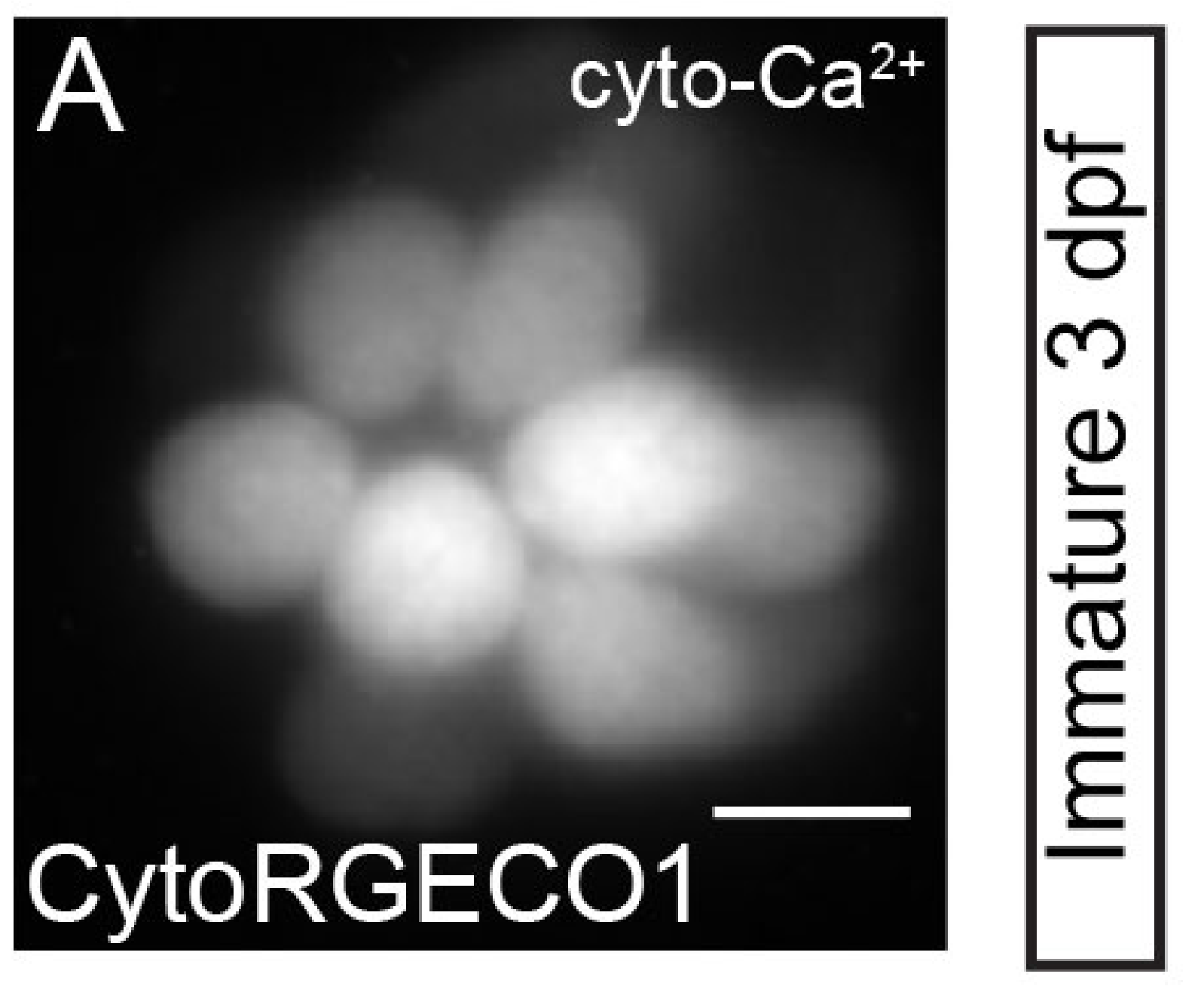

B

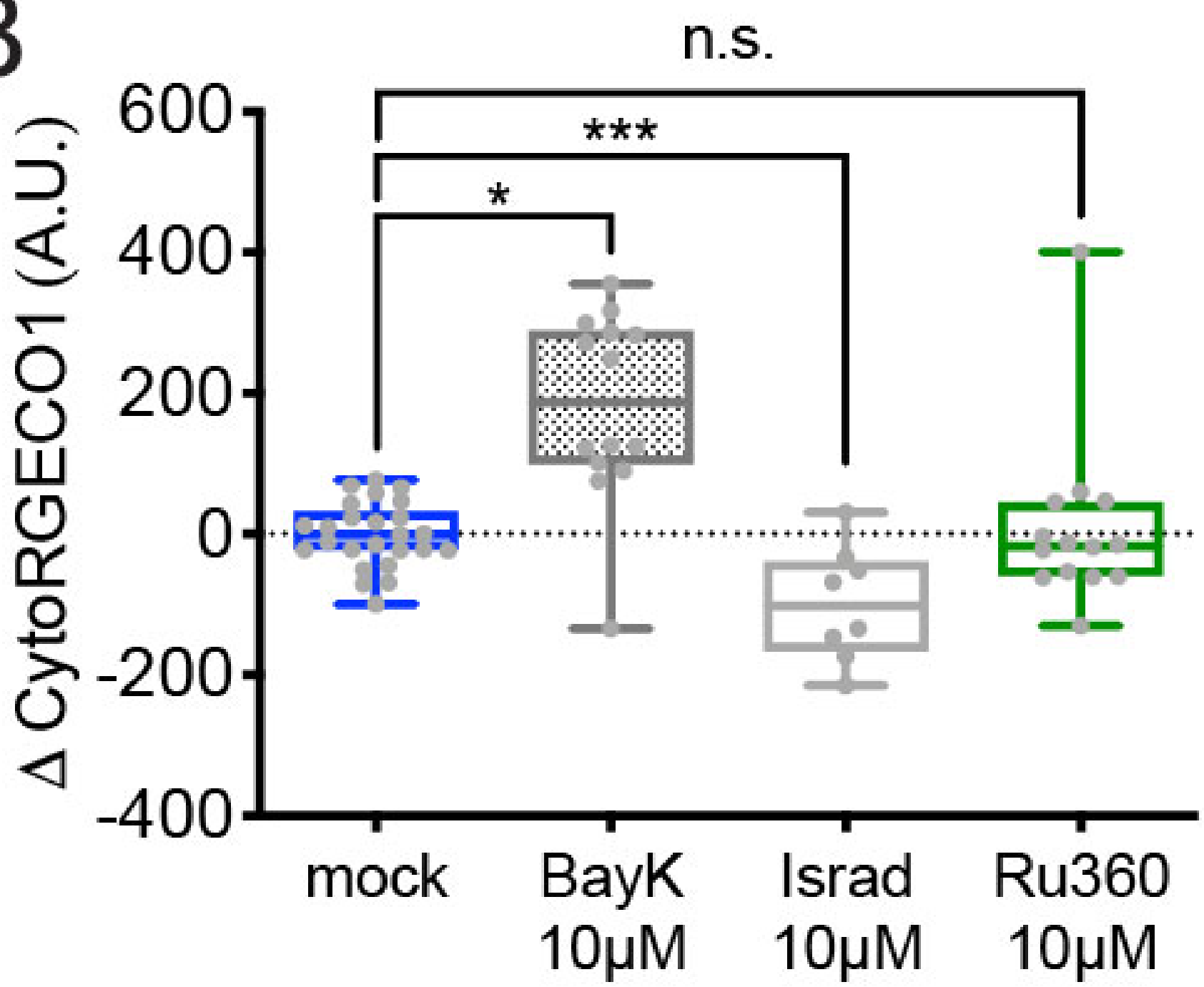

D
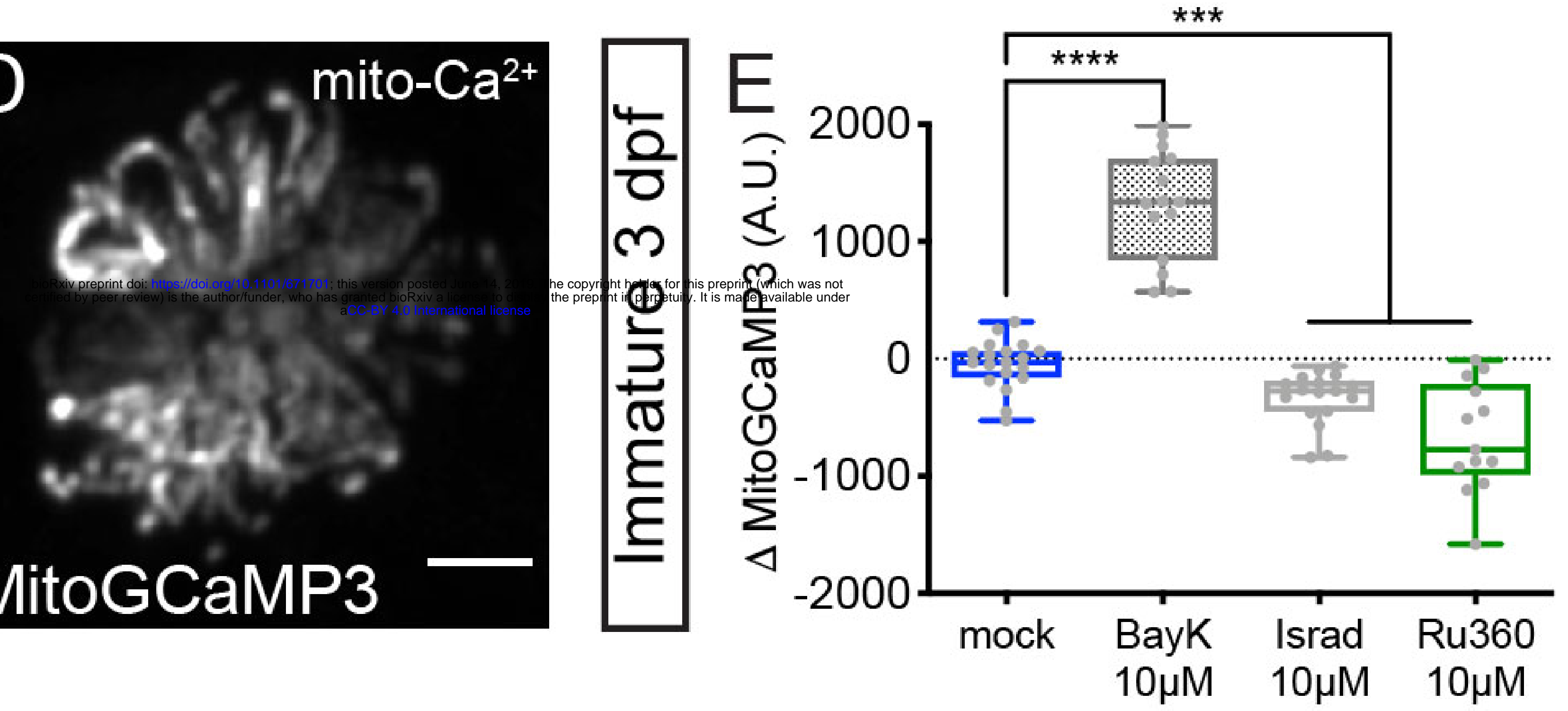

G

$\mathrm{NAD}^{+} / \mathrm{NADH}$

Rex-YFP
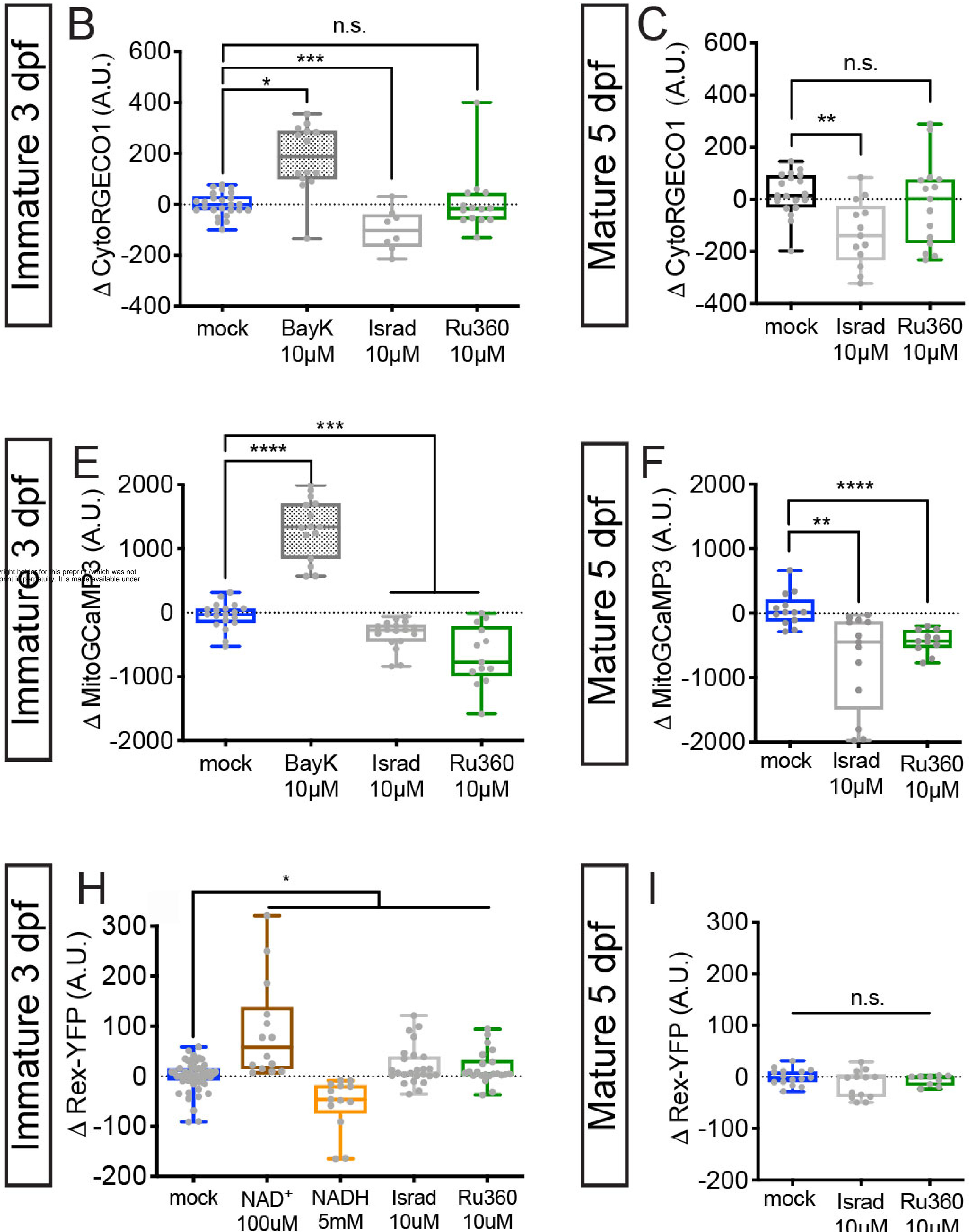
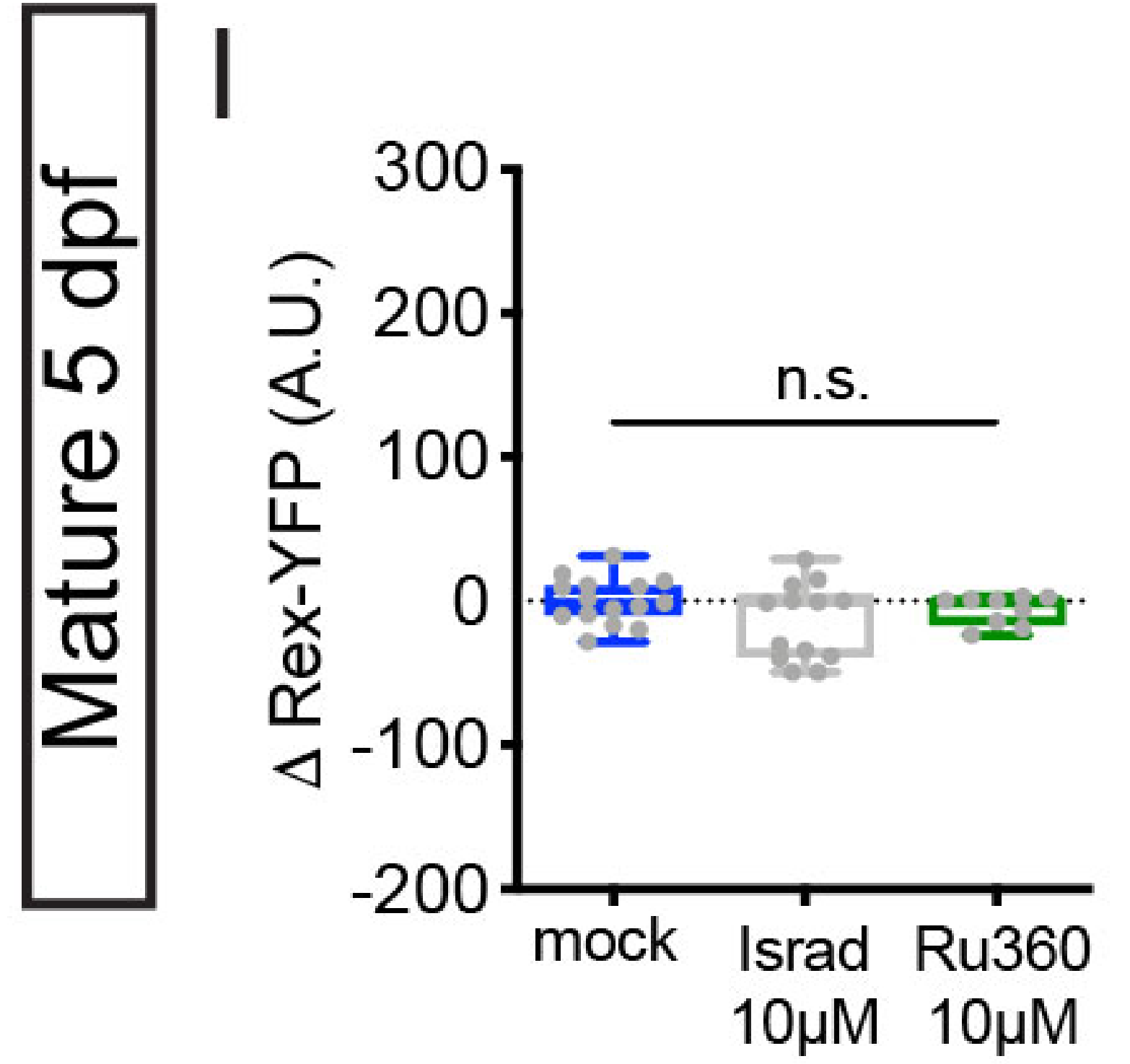
$A$ Developing hair cell

Spontaneous

presynaptic influx

\& Mito-Ca ${ }^{2+}$ uptake

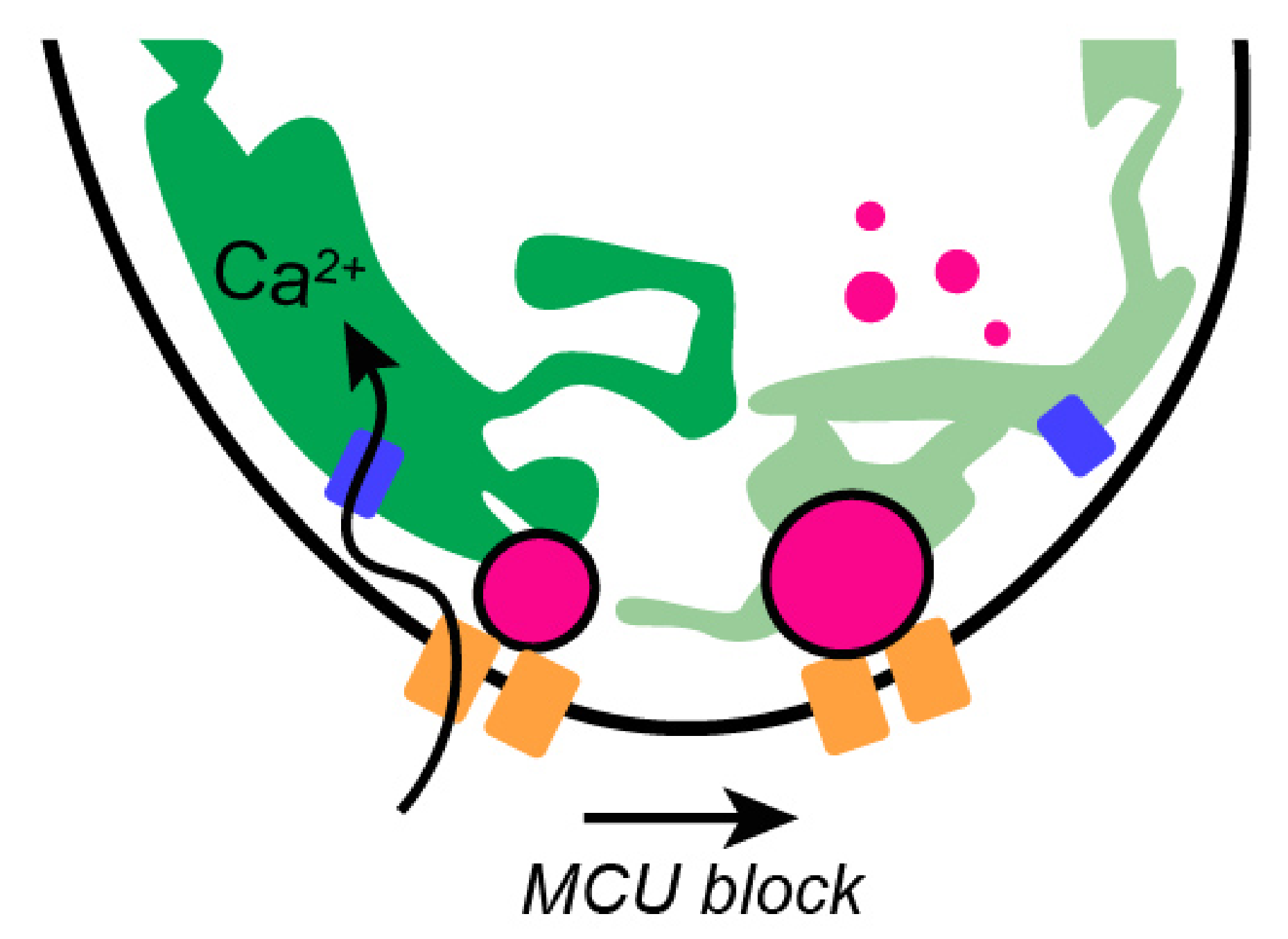

Partial or complete MCU block ribbon enlargement via $N A D(H)$ redox
B Mature hair cell

Evoked

presynaptic influx

\& Mito-Ca ${ }^{2+}$ uptake

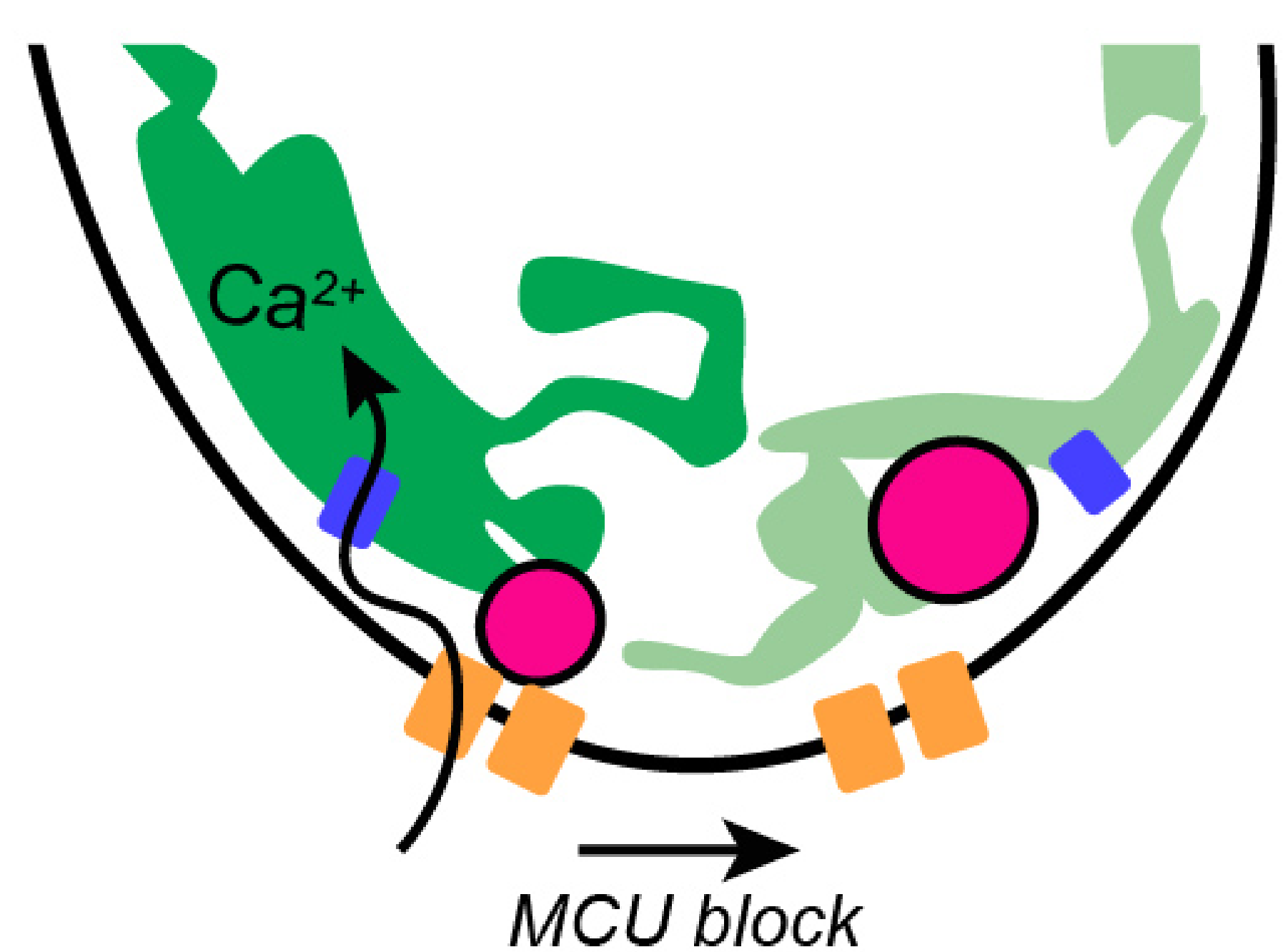

blue $\mathrm{MCU}$

orange $\mathrm{Ca}_{\mathrm{v}} 1.3$

green mitochondria

magenta ribbon/presynapse
Partial MCU block

presynaspe function

loss
Complete MCU block

ribbon enlargement

synapse loss 
A

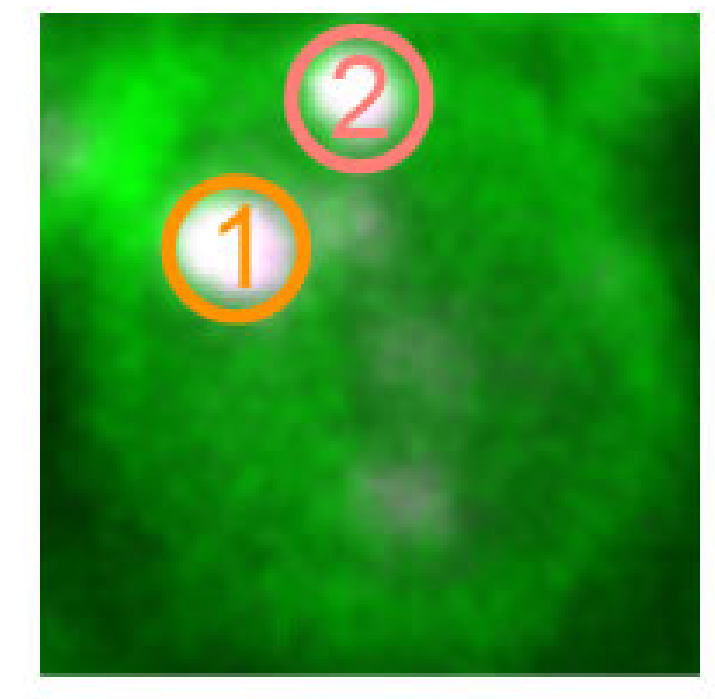

B

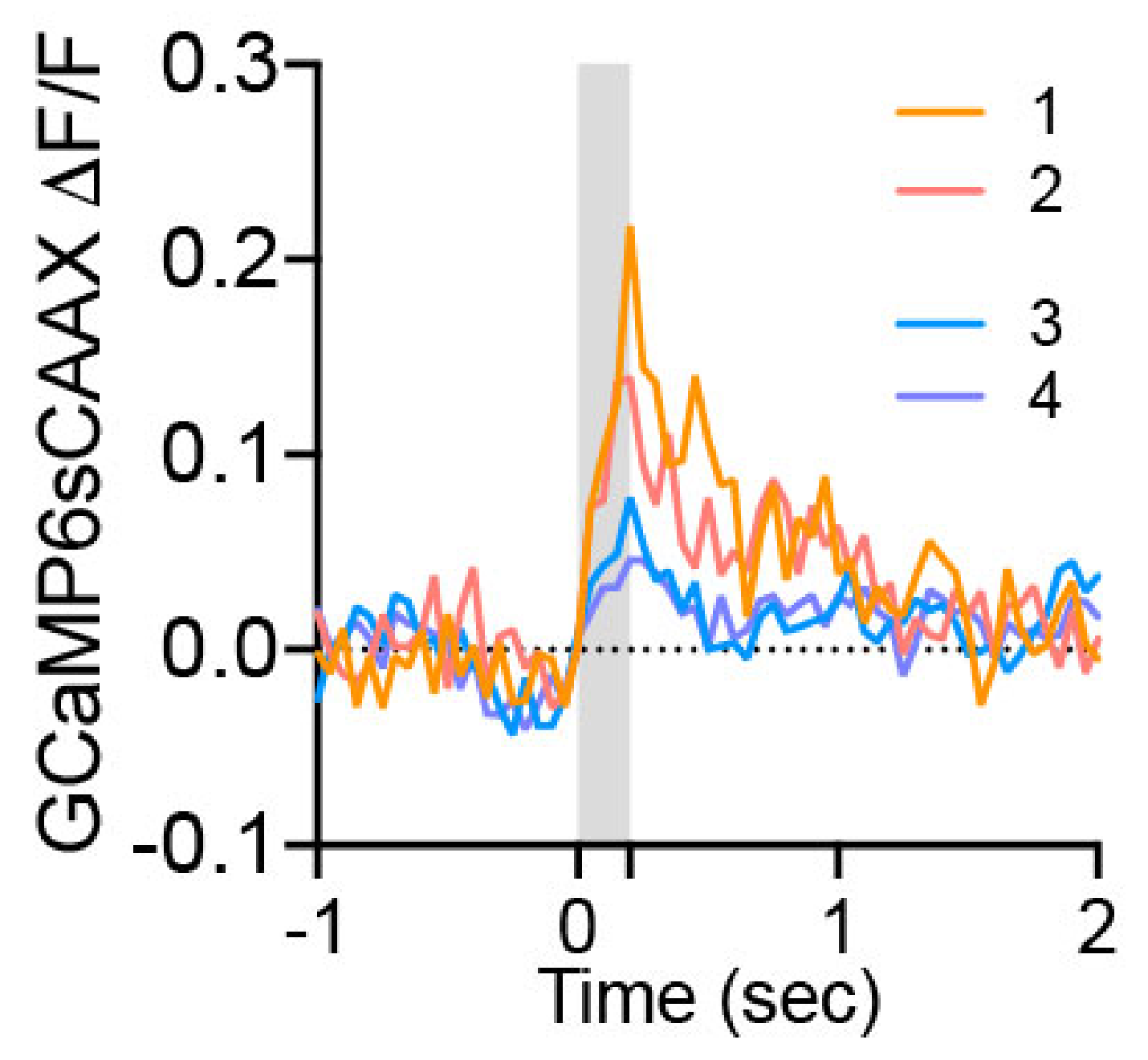




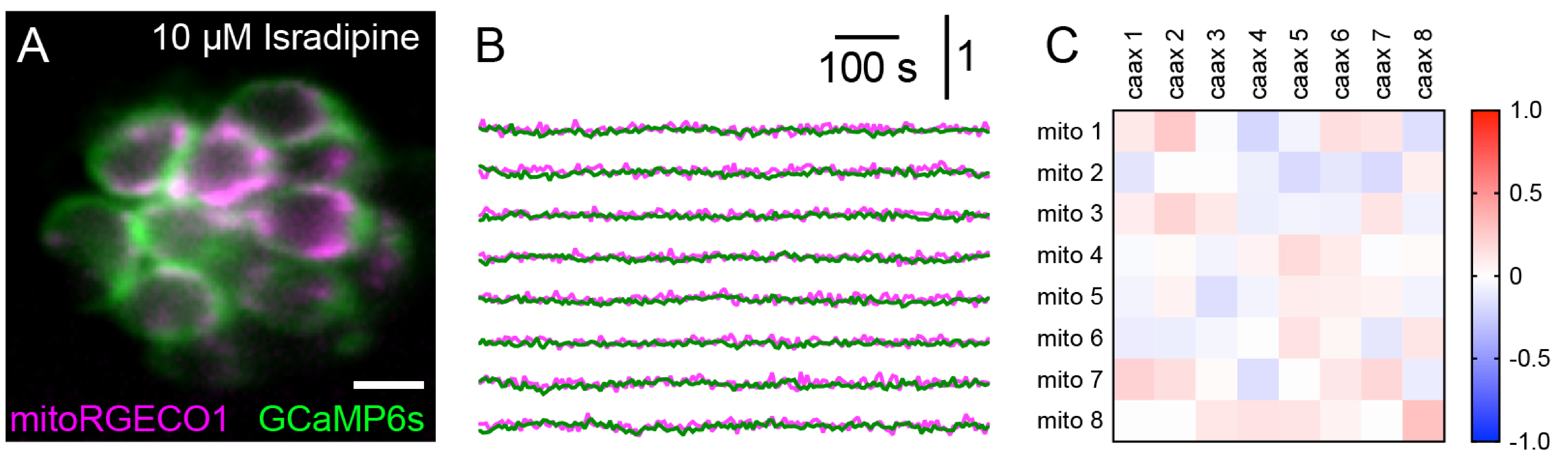

